2. To: (Receiving Organization) Distribution

5. Proj./Prog./Dept./Div.:

Readiness to Proceed/Waste Management/TB\&P/Process Engineering

\section{Originator Remarks:}

This document is being released into the supporting document system for retrievability purposes.

11:-Receiver Remarks: $\cdots \cdots \cdots \cdot 11 \mathrm{~A}$. Design Basel ine Document? [] Yes [X] No For release.
3. From: (originating Organization) Technical Basis and Planning

6. Design Authority/ Design Agent/Cog. Engr.:

M. R. Adams
4. Related EDT No.:

N/A

7: Purchase order No.:

$\mathrm{N} / \mathrm{A}$

9. Equip./Component No.:

$\mathrm{N} / \mathrm{A}$

10. System/B Ldg./Facility: $\mathrm{N} / \mathrm{A}$

12. Major Assm.. Dwg. No. :....

$\mathrm{N} / \mathrm{A}$

13. Permit/Permit Application No.: $\mathrm{N} / \mathrm{A}$

14. Required Response Date: $1 / 19 / 98$

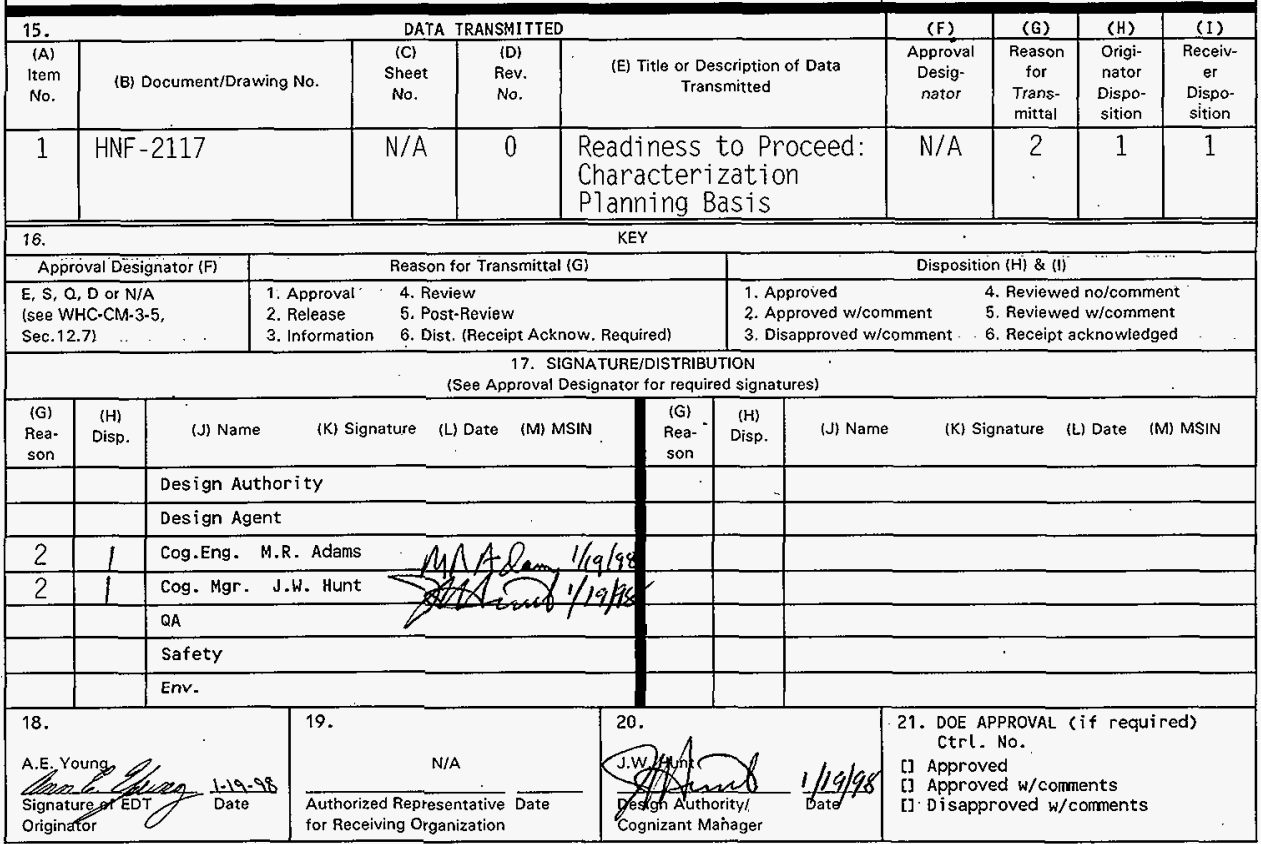




\section{Readiness to Proceed: Characterization Planning Basis}

M. R. Adams

Lockheed Martin Hanford, Corp. , Richland, WA 99352

U.S. Department of Energy Contract DE-AC06-96RL13200

EDT/ECN: EDT-622419

Org Code: 7 A110

B\&R Code: EW 3120074 Total Pages: 96

Key Words: Readiness, Proceed, Characterization. Planning Basis

Abstract: N/A

TRADEMARK DISCLAIMER. Reference herein to any specific commercial product, process, or service by trade name, trademark, manufacturer, or otherwise, does not necessarily constitute or imply its endorsement, recommendation, or favoring by the United States Government or any agency thereof or $i$ ts contractors or subcontractors.

Printed in the United States of America. To obtain copies of this document, contact: Document Control Services, P.O. BoX 950, Mailstop H6-08, Richland WA 99352, Phone (509) 372-2420; Fax $(509) 376-4989$.
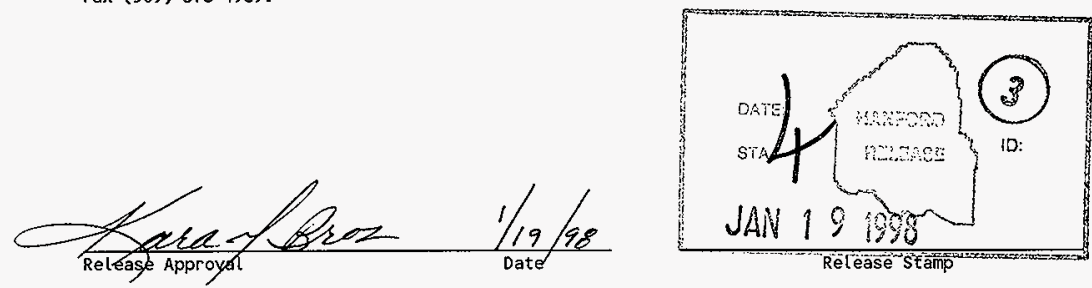
HNF-2117

Revision 0

\section{Readiness to Proceed: Characterization Planning Basis}

Prepared for the U.S. Department of Energy

Assistant Secretary for Environmental Management

Project Hanford Management Contractor for the

U.S. Department of Energy under Contract DE-ACO6-96RL13200

Approved for public release; distribution is unlimited 
HNF-2117

Revision 0

\title{
Readiness to Proceed: Characterization Planning Basis
}

\author{
J. G. Field \\ Lockheed Martin Hanford Corporation \\ Team \\ M. R. Adams \\ J. M. Conner \\ E. I. Husa \\ J. Jo \\ M. J. Kupfer \\ Lockheed Martin Hanford Corporation \\ L. J. Fergestrom \\ Technical Resources, International, Inc.
}

Date Published

January. 1998

Prepared for the U.S. Department of Energy

Assistant Secretary for Environmental Management

Project Hanford. Management Contractor for the

U.S. Department of Energy under Contract DE-AC06-96RL13200

Approved for public release; distribution is unlimited 
HNF-2117 Rev. 0

\section{CONTENTS}

1.0 INTRODUCTION $\ldots \ldots \ldots \ldots \ldots \ldots \ldots \ldots \ldots \ldots \ldots \ldots \ldots \ldots \ldots \ldots \ldots$

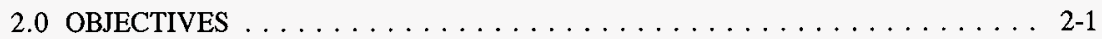

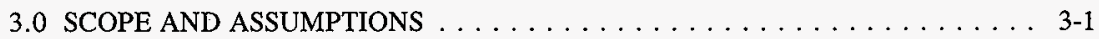

4.0 CHARACTERIZATION REQUIREMENTS

AND AVAILABLE INFORMATION $\ldots \ldots \ldots \ldots \ldots \ldots \ldots . \ldots .4-1$

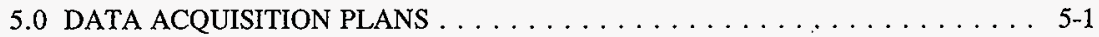

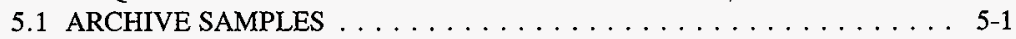

5.2 BEST BASIS ANALYSES $\ldots \ldots \ldots \ldots \ldots \ldots \ldots \ldots \ldots \ldots \ldots$ 5-2

5.3 SAMPLING PLANS . . . . . . . . . . . . . . 5-2

6.0 RECOMMENDATIONS $\ldots \ldots \ldots \ldots \ldots \ldots \ldots \ldots \ldots \ldots \ldots \ldots . \ldots \ldots$

APPENDIX A CHARACTERIZATION DATA REQUIREMENTS . . . . . . . A A-1

APPENDIX B TANK SAMPLING PLANS $\ldots \ldots \ldots \ldots \ldots \ldots \ldots$ B-1 
HNF-2117 Rev. 0

\section{LIST OF TABLES}

4-1 General Characterization Requirements for Readiness to Proceed: Phase I . . . . . . 4-2

4-2 Solid and Liquid Analytical Data Not Available for Phase I Privatization Tanks . . 4-8

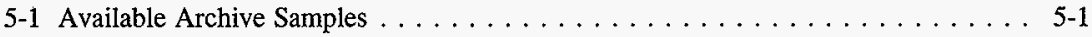

6-1 Summary of Sample Data Not Available and Needs for Tank Privatization . . . . . . 6-1 


\section{READINESS TO PROCEED: CHARACTERIZATION PLANNING BASIS}

\subsection{INTRODUCTION}

This report summarizes characterization requirements, data availability, and data acquisition plans in support of the Phase I Waste Feed Readiness to Proceed Mid-Level Logic. It summarizes characterization requirements for the following program planning documents:

- Waste Feed Readiness Mid-Level Logic and Decomposition (in development)

- Master blue print (not available)

- Tank Waste Remediation System (TWRS) Operations and Utilization Plan (O\&UP) and Privatization Contract

- Enabling assumptions (not available)

- Privatization low-activity waste (LAW) Data Quality Objective (DQO)

- Privatization high-level waste (HLW) DQO (draft)

- Problem-specific DQOs (in development)

- Interface control documents (draft).

Section 2.0 defines the primary objectives for this report, Section 3.0 discusses the scope and assumptions, and Section 4.0 identifies general characterization needs and analyte-specific characterization needs or potential needs included in program documents and charts.

Section 4.0 also shows the analyses that have been conducted, and the archive samples that are available for additional analyses. Section 5.0 discusses current plans for obtaining additional samples and analyses to meet readiness-to-proceed requirements. Section 6.0 summarizes sampling needs based on preliminary requirements and discusses other potential characterization needs.

Many requirements documents are preliminary. In many cases, problem-specific DQOs have not been drafted, and only general assumptions about the document contents could be obtained from the authors. As a result, the readiness-to-proceed characterization requirements provided in this document are evolving and may change. 
HNF-2117 Rev. 0

This page intentionally left blank. 
HNF-2117 Rev. 0

\subsection{OBJECTIVES}

The overall intent of this report is to verify that characterization planning is able to provide the required information to support Phase I planning activities and/or subactivities. Three primary objectives of this report are to:

1. list characterization requirements specified in program planning documents

2. show the characterization information that does exist and determine whether it is sufficient to meet program planning requirements

3. recommend how characterization information can be made available in sufficient time to meet program needs, if it is not available at this time. 
HNF-2117 Rev. 0

This page intentionally left blank. 


\subsection{SCOPE AND ASSUMPTIONS}

Phase I characterization requirements were assessed for the following tanks designated as LAW privatization tanks, HLW privatization tanks, interim feed staging tanks, and feed tanks.

\section{Phase I Low-Activity Waste Privatization Tanks}

241-AN-102, 241-AN-103, 241-AN-104, 241-AN-105, 241-AN-106, 241-AN-107

241-AW-101

241-AZ-101, 241-AZ-102

241-SY-101, 241-SY-103

Phase I High-Level Waste Privatization Tanks

241-AZ-101, 241-AZ-102

241-AY-102

Phase I Privatization Interim Feed Staging Tanks

241-AP-102, 241-AP-104

Phase I Privatization Feed Tanks

241-AP-106, 241-AP-108

Data availability was assessed for solids and supernatant samples. The TWRS O\&UP document identifies waste envelopes for the tanks within the scope and shows what portion of the tank waste (that is, supernatant, soluble portion, or entire tank) will be transferred as part of Phase I retrieval operations. However, no program document clearly indicates whether analytical requirements apply to supernatant, solids, or both.

Although data is available for the current contents of the privatization interim staging and feed tanks, most current waste inventory is expected to be transferred out of these tanks, and waste from designated privatization tanks will be transferred in. Consequently, current analytical information will not meet all readiness-to-proceed needs, and additional sampling and analyses of these tanks may be required after transfers occur. 
HNF-2117 Rev. 0

This page intentionally left blank. 


\subsection{CHARACTERIZATION REQUIREMENTS AND AVAILABLE INFORMATION}

Available program documents were reviewed to determine characterization requirements. Table 4-1 summarizes the general needs, questions, and issues identified in preliminary program documents. Because many documents are in development or are currently being revised, Table 4-1 also shows document status. Table A-1 summarizes analyte-specific characterization program requirements for applicable program documents.

Available analytical data information for each tank was compiled from the Tank Characterization Database (TCD). The data used were obtained after 1989 when the Tri-Party Agreement protocols were established. Data obtained before 1989 are considered unacceptable for making regulatory decisions and were not included in the assessment. The TCD identifies hundreds of types of analyses for each tank and provides results for analyses conducted. In addition to the TCD, data and data assessments are available in Tank Characterization Reports, topical issue reports, and the Surveillance Analysis Computer System (SACS) database.

All designated Phase I LAW and HLW privatization tanks have been sampled, and many analytical needs have been met. Table A-2 identifies and summarizes available data and characterization needs which have not been met for each LAW and HLW tank.

Data for characterization outside the tanks (other than tank waste characterization) were not included in this report because requirements are largely unspecified, and no analyte-specific requirements for characterization outside the tanks are included in the program documents. Many characterization needs outside the tanks (that is, biota, soil samples, raw water, and process water) have not been identified.

Some available information for soils and ground water outside the tank can be obtained from Bechtel or the Pacific Northwest National Laboratory from the Waste Information Data System (WIDS) and ground water monitoring databases. Some biota and ecological survey information may be available in the WIDS and Hanford Environmental Impact Statement documents. 
Table. 4-1. General Characterization Requirements for Readiness to Proceed: Phase I. ( 6 sheets)

\begin{tabular}{|c|c|}
\hline 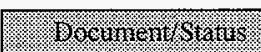 & Resurierients. \\
\hline $\begin{array}{l}\text { Mid-level logic } \\
\text { status: Draft A }\end{array}$ & $\begin{array}{l}\text { May need samples/analyses for environmental and nuclear source } \\
\text { term envelopes. } \\
\text { Do need physical properties of waste (in LAW DQO) to design } \\
\text { tank 241-AN-105 mixing and retrieval system. } \\
\text { Additional analysis may be needed to update the environmental } \\
\text { baseline (i.e., NEPA, TWRS EIS, RCRA, CAA or other permits). } \\
\text { New envelope limits (to be established) may include additional } \\
\text { analytes. Also need to resolve whether envelope applies to bulk } \\
\text { waste or liquid phase only. } \\
\text { MLL and other documents require waste compatibility DQO (see } \\
\text { Table A-1). } \\
\text { Establish Na inventory for LLW tanks, excluding Na and Si; } \\
\text { provide volatile oxides inventory for HLW tanks. }\end{array}$ \\
\hline $\begin{array}{l}\text { MLL decomposition } \\
\text { (not completed) }\end{array}$ & $\begin{array}{l}\text { Breakdown major tasks in the MLL. Project schedule and tasks. } \\
\text { In addition to analytical needs in the MLL, preliminary } \\
\text { decomposition logic specifies the following: RFP envelope } \\
\text { analysis, compatibility DQO, sludge wash testing for tank } \\
\text { 241-AZ-102 (probably similar to analytical/testing requirements for } \\
\text { tank 241-C-106). }\end{array}$ \\
\hline $\begin{array}{l}\text { Master blueprint } \\
\text { (not completed) }\end{array}$ & $\begin{array}{l}\text { The master blueprint is expected to provide an overall picture of the } \\
\text { Phase I Readiness-to-Proceed Privatization project (impact } \\
\text { requirements are unknown). }\end{array}$ \\
\hline
\end{tabular}


Table. 4-1. General Characterization Requirements for Readiness to Proceed: Phase I. (6 sheets)

\begin{tabular}{|c|c|}
\hline 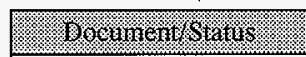 & Regivitebrents. \\
\hline $\begin{array}{l}\text { TWRS O\&UP } \\
\text { (Rev. 0) and } \\
\text { Privatization } \\
\text { Contract }\end{array}$ & $\begin{array}{l}\text { Lists analytical requirements for waste delivery processes and plans } \\
\text { (see Table A-1). All LAW feed will meet tank farm specifications } \\
\text { in OSD-T-151-0007. All feed will have maximum 5 vol. \% solids. } \\
\text { Table 3.1-8 shows the projected LAW feed processing sequence. It } \\
\text { specifies that Envelope A applies to tanks 241-AW-101, } \\
\text { 241-AN-105, 241-AN-104 and 241-AN-103. Envelope B applies to } \\
\text { tank 241-AZ-101 and 241-AZ-102. Envelope C applies to } \\
\text { 241-AN-102, 241-AN-106, 241-AN-107, 241-SY-101 and } \\
\text { 241-SY-103. Supernatant only will be removed from } \\
\text { tanks 241-AZ-101, 241-AZ-102, 241-AN-102, 241-AN-106 and } \\
\text { 241-AN-107. Sludge washing tests for tanks 241-AZ-101, } \\
\text { 241-AZ-102, and 241-AY-102 may be required. }\end{array}$ \\
\hline $\begin{array}{l}\text { Privatization LAW } \\
\text { DQO, Rev. 0. (Rev. } 1 \\
\text { is in progress). } \\
\end{array}$ & $\begin{array}{l}\text { Includes analyte-specific requirements and defined envelopes for } \\
\text { LAW (see Table A-1): }\end{array}$ \\
\hline $\begin{array}{l}\text { Privatization HLW DQO } \\
\text { (being drafted) }\end{array}$ & $\begin{array}{l}\text { Expected to include a list of reportable analytes (see Table A-1), } \\
\text { solids screening tests to evaluate solubility, organics, and physical } \\
\text { properties. Envelope D will apply to HLW feed. }\end{array}$ \\
\hline 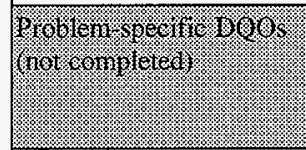 & 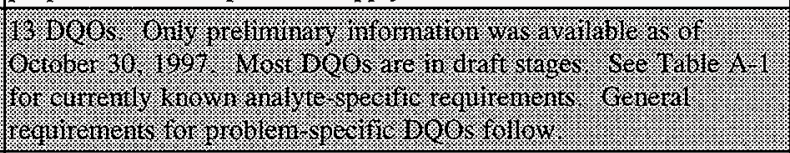 \\
\hline $\begin{array}{l}\text { Confirm Tank T is } \\
\text { appropriate for Batch } \mathrm{X} \\
\text { (LAW) (not completed) }\end{array}$ & $\begin{array}{l}\text { Documents decision process for selecting LAW feed tank. The } \\
\text { scope encompasses the following. 1) Laboratory process testing } \\
\text { using approximately } 1 \text { L of a representative core or tank composite. } \\
\text { Testing includes physical properties and water wash behavior. } \\
\text { 2) Statistical evaluation of chemical data for comparison to waste } \\
\text { envelope limits. If tank contents are near the limits (within } \\
20 \text { percent) or uncertainty is high, additional sampling and/or } \\
\text { analyses may be necessary. Early indications are that analysis of } \\
\text { existing core composites (that is, archive samples) will be } \\
\text { satisfactory if additional data are needed. }\end{array}$ \\
\hline $\begin{array}{l}\text { Confirm Tank } \mathrm{T} \text { is } \\
\text { appropriate for Batch X } \\
\text { (HLW) (not completed) }\end{array}$ & $\begin{array}{l}\text { Documents decision process for selecting tank for HLW feed } \\
\text { staging. } \\
\text { No sampling or analytical requirements are specified. Similar to } \\
\text { requirements for LAW Tank T. }\end{array}$ \\
\hline
\end{tabular}


Table. 4-1. General Characterization Requirements for Readiness to Proceed: Phase I. (6 sheets)

\begin{tabular}{|c|c|}
\hline Dectininert Stakus & Requiranemts: \\
\hline $\begin{array}{l}\text { Equipment design } \\
\text { (rough draft) }\end{array}$ & 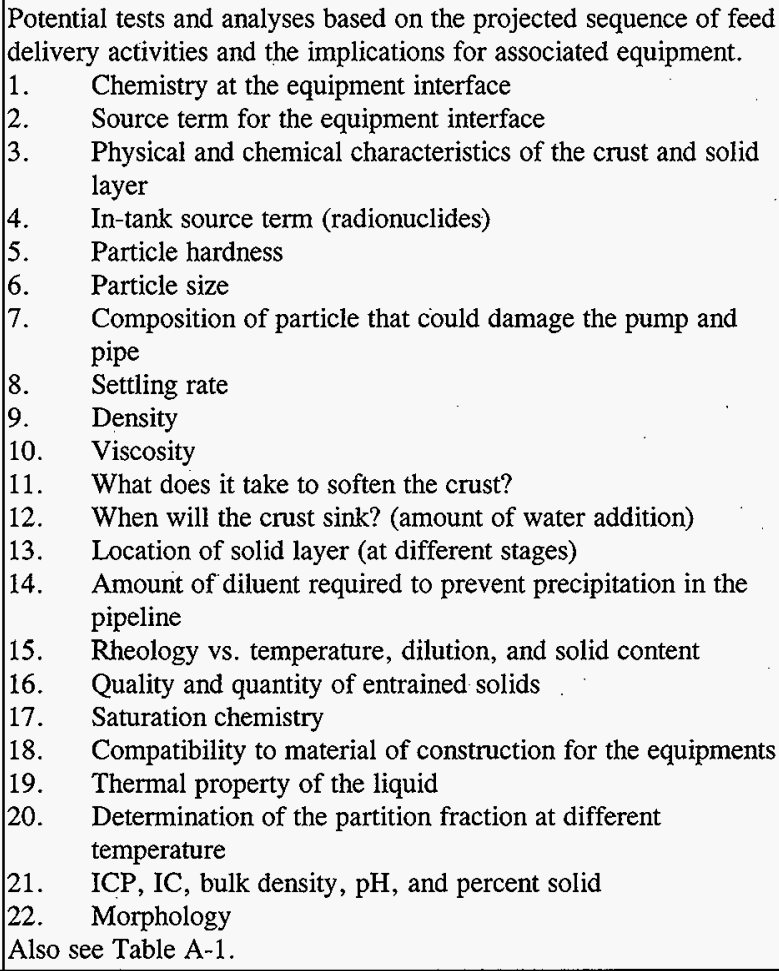 \\
\hline Safety Basis & No analytical requirements \\
\hline $\begin{array}{l}\text { Waste Feed Delivery } \\
\text { Permitting }\end{array}$ & $\begin{array}{l}\text { Requirements listed in Table A-1 based on } \\
\text { WHC-SD-WM-DQO-025, DQOs for Regulatory Requirements for } \\
\text { Dangerous Waste Sampling and Analysis and } \\
\text { WHC-SD-WM-DQO-021, DQOs for Regulatory Requirements for } \\
\text { Hazardous and Radioactive Air Emissions Sampling and Analysis. }\end{array}$ \\
\hline $\begin{array}{l}\text { DST Inventory } \\
\text { Configuration Control }\end{array}$ & $\begin{array}{l}\text { No analytical requirements. This DQO states that the compatibility } \\
\text { DQO applies. }\end{array}$ \\
\hline
\end{tabular}


Table. 4-1. General Characterization Requirements for Readiness to Proceed: Phase I. (6 sheets)

\begin{tabular}{|c|c|}
\hline 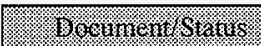 & 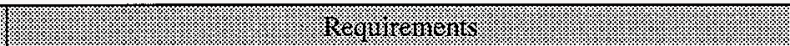 \\
\hline $\begin{array}{l}\text { In Tank Processing } \\
\text { (Process Control) }\end{array}$ & $\begin{array}{l}\text { The process control DQO will dictate the sampling and analyses } \\
\text { necessary to ensure that adjusted batches are likely to fall within } \\
\text { LAW envelope limits. No sampling is possible at this time, and no } \\
\text { current characterization data are applicable. Candidate feed tanks } \\
\text { will be sampled for process control after decanting pumpable } \\
\text { supernatant and diluting, dissolving solids, and mixing the tank. } \\
\text { Three samples will be taken from a single riser. Analyses will be } \\
\text { limited to ICP metals, bulk density, pH, and percent solids. } \\
\text { Process control samples also will be taken in the intermediate feed } \\
\text { staging tanks (241-AP-102 and -104). Again, sampling is expected } \\
\text { to consist of three samples from one riser. Analyses will be limited } \\
\text { to ICP metals, bulk density, pH, and percent solids. Analyses must } \\
\text { be completed in a short time period. }\end{array}$ \\
\hline $\begin{array}{l}\text { Waste Transfer } \\
\text { (Delivery) to Private } \\
\text { Contractors (LAW) }\end{array}$ & $\begin{array}{l}\text { No sampling is possible at this time, and current characterization } \\
\text { data may not be applicable. One sampling event is necessary per } \\
\text { intermediate feed staging tank (241-AP-102 or }-104) \text { per batch. } \\
\text { A reasonable estimate of the sampling necessary is six grab samples } \\
\text { (bottle-on-string) per sampling event. } \\
\text { Analytical requirements are limited to the analytes stated in the } \\
\text { privatization contract. These are the same as the LAW } \\
\text { Privatization DQO Group I analytes. These analytes }(20-30) \\
\text { include metals, anions, TOC, OH, }{ }^{137} \mathrm{Cs},{ }^{90} \mathrm{Sr},{ }^{99} \mathrm{Tc} \text {, and TRU. } \\
\text { However, the contracts may be renegotiated (the list of analytes and } \\
\text { the ratios to sodium may change). } \\
\text { This DQO will describe analytical procedures and data analyses as } \\
\text { they differ from standard protocols. The protocol for establishing } \\
\text { Na content in the batch will be specified. }\end{array}$ \\
\hline $\begin{array}{l}\text { Waste Transfer } \\
\text { (Delivery. to Private } \\
\text { Contractors (HLW) }\end{array}$ & $\begin{array}{l}\text { The DQO will require sampling of the feed tank to evaluate } \\
\text { whether waste meets envelope D specifications. Envelope D } \\
\text { requirements are specified in the TWRS O\&UP but may be } \\
\text { modified when the Privatization HLW DQO is completed. }\end{array}$ \\
\hline $\begin{array}{l}\text { ILAW storage and } \\
\text { disposal }\end{array}$ & To be canceled \\
\hline HLW interim storage & To be canceled \\
\hline
\end{tabular}


Table. 4-1. General Characterization Requirements for Readiness to Proceed: Phase I. ( 6 sheets)

\begin{tabular}{|c|c|}
\hline reforine & Realiniementis: \\
\hline $\begin{array}{l}\text { Single-shell tank } \\
\text { sequencing }\end{array}$ & $\begin{array}{l}\text { Information from previous sampling and analyses will be used. No } \\
\text { new tank analysis will be directed by this DQO. }\end{array}$ \\
\hline Model validation & Not completed, preliminary requirements listed in Table A-1. \\
\hline 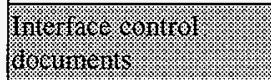 & 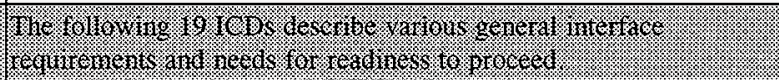 \\
\hline $\begin{array}{l}\text { ICD-01 } \\
\text { Raw Water }\end{array}$ & $\begin{array}{l}\text { Testing, appears to be mostly flow rate tests, may include } \\
\text { composition and laboratory analyses. }\end{array}$ \\
\hline \begin{tabular}{|l|} 
ICD-02 \\
Potable Water
\end{tabular} & $\begin{array}{l}\text { Water flushing, testing, disinfection and disposal. May include } \\
\text { some laboratory analysis to verify water meets drinking water } \\
\text { standards. }\end{array}$ \\
\hline $\begin{array}{l}\text { ICD-03 } \\
\text { Radiation Solid Waste }\end{array}$ & $\begin{array}{l}\text { Need to certify as radiation. Waste? } \\
\text { Waste disposal analysis required? }\end{array}$ \\
\hline $\begin{array}{l}\text { ICD-05 } \\
\text { Nonradiation. No } \\
\text { dangerous effluent. }\end{array}$ & $\begin{array}{l}\text { Verify volume and composition of effluent discharged. } \\
\text { Verify "Non-Rad" for } 200 \text { Area TEDF to accept effluent. } \\
\text { TEDF permit modification analytical support may be required. }\end{array}$ \\
\hline $\begin{array}{l}\text { ICD-06 } \\
\text { Radiation. Dangerous } \\
\text { Effluent }\end{array}$ & $\begin{array}{l}\text { Verify volume and composition of effluent discharged. } \\
\text { Characterize per Liquid Effluent Retention Facility/Waste analysis } \\
\text { plan permit. } \\
\text { Analytical data for preliminary screening of liquid or to identify } \\
\text { regulatory and environmental concerns? }\end{array}$ \\
\hline $\begin{array}{l}\text { ICD-09 } \\
\text { Land for Siting }\end{array}$ & $\begin{array}{l}\text { Establish baseline site and environmental conditions for NEPA, } \\
\text { cultural, biological, seismic, soil and groundwater contamination } \\
\text { per environmental checklist. } \\
\text { Analytical support to Site Evaluation Reports. } \\
\text { Analysis of artifacts or cultural sites. }\end{array}$ \\
\hline $\begin{array}{l}\text { ICD-10 } \\
\text { Deactivated } \\
\text { Facility and Site } \\
\end{array}$ & $\begin{array}{l}\text { RCRA closure plan may require analytical support. } \\
\text { Verify closed site meets regulatory requirements. }\end{array}$ \\
\hline $\begin{array}{l}\text { ICD-11,12 } \\
\text { Electricity, } \\
\text { roads and rails }\end{array}$ & $\begin{array}{l}\text { No characterization requirements specified for electricity. May } \\
\text { have siting needs for new roads/rails. }\end{array}$ \\
\hline
\end{tabular}


Table. 4-1. General Characterization Requirements for Readiness to Proceed: Phase I. (6 sheets)

\begin{tabular}{|l|l|}
\hline \multicolumn{1}{|l|}{$\begin{array}{l}\text { Deding } \\
16,17,18\end{array}$} & $\begin{array}{l}\text { May need tests to verify composition before shipping waste } \\
\text { samples? } \\
\text { Smears and radioactive monitoring of waste and containers. } \\
\text { Dose rate monitoring of waste packages. } \\
\text { For }{ }^{137} \text { Cs intermediate product, drop test required to put it in the } \\
\text { Interim Storage facility? } \\
\text { Vitrified cesium analyses? }\end{array}$ \\
\hline $\begin{array}{l}\text { ICD-19,20 } \\
\text { LAW and HLW waste } \\
\text { feed }\end{array}$ & $\begin{array}{l}\text { ICD-19 includes A, B, and C Envelope requirements for } \\
\text { nonradionuclides. ICD-20 includes HLW waste envelope limits for } \\
\text { radionuclides, nonradionuclides, and physical properties. Same as } \\
\text { the TWRS O\&UP. }\end{array}$ \\
\hline $\begin{array}{l}\text { ICD-21 } \\
\text { Waste feed tank } \\
\text { support system } \\
\text { LAW }\end{array}$ & $\begin{array}{l}\text { May have analytical siting requirements } \\
\text { Monitoring during interface: corrosion, heat generation, tank level, } \\
\text { master pump shutdown. } \\
\text { Tank ventilation monitoring? }\end{array}$ \\
\hline $\begin{array}{l}\text { ICD-22 } \\
\text { Air Emissions }\end{array}$ & $\begin{array}{l}\text { Analysis to comply with regulatory requirements } \\
\text { and prepare permits and reports } \\
\text { (i.e., Air Operating Permit, Treatment Storage and Disposal, } \\
\text { Emergency Planning and Community Right-to-Know Act). }\end{array}$ \\
\hline
\end{tabular}

Notes:

$\begin{array}{lll}\text { CAA } & = & \text { Civil Aeronautics Administration } \\ \text { EIS } & = & \text { Environmental Impact Study } \\ \text { IC } & = & \text { Ion chromatography } \\ \text { ICP }= & \text { Inductively coupled plasma } \\ \text { MLL }= & \text { mid-level logic } \\ \text { RCRA }= & \text { Resource Conservation and Recovery Act } \\ \text { RFP }= & \text { Request for Proposal } \\ \text { TOC }= & \text { total organic carbon } \\ \text { TRU }= & \text { Transuranic }\end{array}$


Table 4-2. Solid and Liquid Analytical Data Not Available for Phase I Privatization Tanks. (2 sheets)

\begin{tabular}{|c|c|c|}
\hline 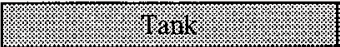 & Solvos & 1.rauras \\
\hline 241-AN-102 & No solids samples were taken. & $\begin{array}{l}\text { Most ICP and IC, organics, } \\
\text { physical properties, and most } \\
\text { radionuclides. }\end{array}$ \\
\hline 241-AN-103 & $\begin{array}{l}\text { Citrate, HEDTA, ammonia, } \\
\text { carbonate, } \mathrm{Cs}, \mathrm{Hg}, \mathrm{Te}, \mathrm{Cr}^{+6} \text {, } \\
\text { many organics, particle size } \\
\text { distr., pH, settled solids, shear } \\
\text { strength, viscosity, vol. \% settled } \\
\text { solids, many radionuclides. } \\
\end{array}$ & Same as solids. \\
\hline 241-AN-104 & $\begin{array}{l}\text { Citrate, HEDTA, ammonia, } \\
\text { carbonate, } \mathrm{Cs}, \mathrm{Hg}, \mathrm{Te}, \mathrm{Cr}^{+6} \text {, } \\
\text { many organics, particle size } \\
\text { distr., pH, settled solids, shear } \\
\text { strength, viscosity, vol. \% settled } \\
\text { solids, many radionuclides. }\end{array}$ & Same as solids. \\
\hline 241-AN-105 & $\begin{array}{l}\text { Citrate, HEDTA, ammonia, } \\
\text { carbonate, } \mathrm{Cs}, \mathrm{Hg}, \mathrm{Pd}, \mathrm{Te}, \mathrm{Cr}^{+6} \text {, } \\
\text { many organics, particle size } \\
\text { distr., pH, settled solids, shear } \\
\text { strength, viscosity, vol. \% settled } \\
\text { solids, many radionuclides. }\end{array}$ & Same as solids. \\
\hline \begin{tabular}{|l|}
$241-A N-106$ \\
(transferring supernate only)
\end{tabular} & No solids samples were taken. & $\begin{array}{l}\text { Need all required analyses } \\
\text { except percent water, gross } \\
\text { alpha, DSC, total organic } \\
\text { carbon and SpG. }\end{array}$ \\
\hline $\begin{array}{l}241-\mathrm{AN}-107 \\
\text { (transferring supernate only) }\end{array}$ & No solids samples were taken. & $\begin{array}{l}\text { Need all required analyses } \\
\text { except percent water, gross } \\
\text { alpha, DSC, total organic } \\
\text { carbon and SpG. }\end{array}$ \\
\hline 241-AW-101 & $\begin{array}{l}\text { Citrate, formate, HEDTA, } \\
\text { ammonia, carbonate, hydroxide, } \\
\text { Cs, Hg, Te, many organics, } \\
\text { particle size distr., pH, settled } \\
\text { solids, shear strength, viscosity, } \\
\text { vol. \% settled solids, many } \\
\text { radionuclides. }\end{array}$ & Same as solids. \\
\hline
\end{tabular}


Table 4-2. Solid and Liquid Analytical Data Not Available for Phase I Privatization Tanks.

\begin{tabular}{|c|c|c|}
\hline T: Tark & d & 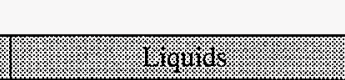 \\
\hline 241-AY-102 & No solids samples were taken. & $\begin{array}{l}\text { Cyanide, ammonia, } \\
\text { carbonate, many ICP metals, } \\
\text { many organics, physical } \\
\text { properties except } \mathrm{SpG} \text {, many } \\
\text { radionuclides. }\end{array}$ \\
\hline 241-AZ-101 & $\begin{array}{l}\text { No solids samples were taken } \\
\text { after } 1989 \text { (May } 1989 \text { core). }\end{array}$ & $\begin{array}{l}\text { Bromide, citrate, formate, } \\
\text { HEDTA, ammonia, } \\
\text { carbonate, } \mathrm{Cr}^{+6}, \text { many ICP } \\
\text { metals, many organics, } \\
\text { physical properties except } \\
\text { SpG, many radionuclides. }\end{array}$ \\
\hline $\begin{array}{l}241-\mathrm{AZ}-102 \\
\text { (transferring supernate only. }\end{array}$ & $\begin{array}{l}\text { No solids samples were taken } \\
\text { after } 1989 \text { (May } 1989 \text { core). }\end{array}$ & $\begin{array}{l}\text { Bromide, citrate, formate, } \\
\text { HEDTA, ammonia, } \\
\text { carbonate, } \mathrm{Cr}^{+6}, \text { many ICP } \\
\text { metals, many organics, } \\
\text { physical properties except } \\
\text { SpG, many radionuclides. }\end{array}$ \\
\hline $241-S Y-101$ & $\begin{array}{l}\text { Citrate, formate, HEDTA, } \\
\text { ammonia, several ICP metals, } \\
\text { many organics, many } \\
\text { radionuclides. }\end{array}$ & Same as solids. \\
\hline $241-S Y-103$ & $\begin{array}{l}\text { Citrate, HEDTA, ammonia, } \\
\text { carbonate, many ICP metals, } \\
\text { many organics, particle size } \\
\text { distr., settled solids, shear } \\
\text { strength, viscosity, vol. \% settled } \\
\text { solids, many radionuclides. }\end{array}$ & \\
\hline $\begin{array}{l}241-A P-102,-104,-106 \\
-108\end{array}$ & $\begin{array}{l}\text { New samples may be needed after } \\
\text { waste transfers. }\end{array}$ & $\begin{array}{l}\text { New samples may be needed } \\
\text { after waste transfers. }\end{array}$ \\
\hline
\end{tabular}


HNF-2117 Rev. 0

This page intentionally left blank. 


\subsection{DATA ACQUISITION PLANS}

\subsection{ARCHIVE SAMPLES}

Where possible, archive samples can be analyzed to obtain needed data. Table 5-1 shows the estimated amount and types of archive samples for each tank in the scope, based on information available in the tank archive database. These values should be considered approximate until, and if, a physical inventory of the sample archive is performed.

"Solids" vials may contain saltcake, sludge, or saltcake and sludge and may be centrifuged solids or uncentrifuged. Methods for separating liquids from solids varied. In many cases, liquids were skimmed from the top of a sample after suspended solids settled to the bottom of a container. The content of a vial is considered "uncertain" if the vial was subsampled from a parent vial, is a composite, or the description is such that the nature of the sample cannot be determined. Laboratory reports for applicable samples should be consulted to determine the origin of the archive samples and the method of preparation.

The archive vials will need to be checked to determine whether enough material is available to do a full suite of analyses with duplicates and other quality control samples (for example, $100 \mathrm{ml}$ of liquids or $30 \mathrm{~g}$ of solids). Archive samples will probably not be acceptable for semivolatile and volatile analyses because of the short sample retention time required for these analyses. In addition, some physical tests may require additional sampling to obtain larger sample quantities or "undisturbed" samples.

Table 5-1. Available Archive Samples.

\begin{tabular}{|c|c|c|c|c|}
\hline $\begin{array}{ll}1 & \\
\end{array}$ & $\begin{array}{l}\text { Terat Yals } \\
\text { A-Bf? }\end{array}$ & 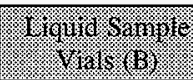 & 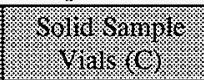 & $\begin{array}{l}\text { Whedrant Vial } \\
\text { Content }\end{array}$ \\
\hline 241-AN-103 & 7 & 0 & 0 & 7 \\
\hline 241-AN-103 & 95 & 14 & 25 & 56 \\
\hline 241-AN-104 & 84 & 22 & 12 & 50 \\
\hline 241-AN-105 & 97 & 32 & 10 & 55 \\
\hline $241-\mathrm{AN}-106$ & 0 & 0 & 0 & 0 \\
\hline 241-AN-107 & 14 & 0 & 0 & 14 \\
\hline 241-AW-101 & 110 & 27 & 34 & 49 \\
\hline 241-AZ-101 & 0 & 0 & 0 & 0 \\
\hline 241-AZ-102 & 0 & 0 & 0 & 0 \\
\hline 241-SY-101 & 0 & 0 & 0 & 0 \\
\hline 241-SY-103 & 28 & 8 & 5 & 15 \\
\hline
\end{tabular}




\subsection{BEST BASIS ANALYSES}

Where archive data cannot be used, another alternative is to determine analytical information based on "best basis" tank inventories. A proposal for using best basis inventory information is included in the draft DQO Confirm Tank $T$ is an Appropriate Feed Source for Low-Activity Waste Feed Batch $X$. Tank best basis inventories have been developed for each LAW and HLW tank and all 177 double-shell and single-shell tanks in the Hanford tank farms. The best basis inventory is based on sample data, engineering assessments, or Hanford Defined Waste model estimates depending on available information. Engineering estimates evaluate tank surveillance data, tank process transfer records, and sample data from tanks containing the same waste types or similar process histories.

Best basis evaluations are available on the Tank Characterization Database and are included as an Appendix in Tank Characterization Reports.

Using best basis values for some required data gaps will probably require approval by regulatory agencies. If best basis evaluations are determined to be insufficient to fill data gaps, then additional samples will have to be taken.

\subsection{SAMPLING PLANS}

If archive samples are not available for required analyses, and if best basis data are not available or not acceptable, additional sampling is required.

Sampling plans are developed according to the Waste Information Requirements Document which identifies tank characterization activities needed for safe storage, retrieval, and disposal of TWRS tank waste. Characterization planning and scheduling tools include the technical sampling priority basis document, baseline sampling schedule, laboratory analysis schedule, tank characterization report schedule, and associated supporting documents.

Table B-1 shows a schedule (as of October 30, 1997) for obtaining many of the needed tank samples for the tanks in scope. Start and finish dates depend on available resources and sampling priority. Sampling dates are revised frequently and sampling will be conducted according to the most current revision of the Baseline Sampling Schedule as prepared by the TWRS characterization program.

After sampling, samples are sent to the 222-S Laboratory for analysis. Standard analyses to address tank issues and quality control checks are scheduled to be completed within 140 days after receiving samples. Data is then loaded into the TCD and is available on the Internet within seven days after the final data report is completed. 


\subsection{RECOMMENDATIONS}

Analytical and physical tests are needed on samples for the tanks within the scope to meet current Phase I privatization requirements specified in program documents, drafts for future program documents, and problem-specific DQOs. Many analyses have already been completed or can be performed with current archive samples and laboratory equipment.

Table 6-1 summarizes the data not available, available archive samples, sample plans, and potential data needs for characterizing tank waste based on preliminary Phase I analytical requirements as of November 1, 1997.

Samples are planned and/or archive material may be available to meet many current characterization requirements. However, to obtain organic analyses for solids, if required, tanks 241-AN-107, 241-AW-101 and 241-AY-102 will need to be resampled or scheduled grab samples will need to include tank solids. Sampling for solids and liquids is needed but is not scheduled for tank 241-SY-101. New samples may also be needed for all tanks within the scope if "undisturbed samples" are required for physical properties analyses. Where it is available, archive material can be used for other analytical needs.

Additional laboratory equipment and/or laboratory facilities may be needed because some required analyses do not appear to be currently available from any onsite laboratories, and it is questionable whether onsite laboratories will be able to meet the production needs for the privatization program. Most analyses are currently performed by the 222-S Laboratory. Organic speciation and some physical analyses are performed mostly by the Pacific Northwest National 325 Laboratory. Many radionuclide and organic analytes listed as requirements and some physical tests have no procedures established and are not currently performed in an onsite laboratory.

As stated previously, this report does not include a discussion of characterization requirements and needs outside the tank. This is because few analyte specific requirements have been determined, and samples and analyses outside the tank have not been scheduled. Table 4-1 identifies many potential general requirements for samples and analyses outside the tanks. These requirements should be assessed further, and a schedule should be established. 
Table 6-1. Summary of Sample Data Not Available and Needs for Tank Privatization. (3 sheets)

\begin{tabular}{|c|c|c|c|c|}
\hline U: Iratil & 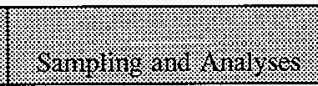 & S Sarininging: & 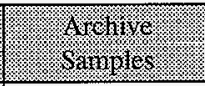 & 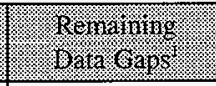 \\
\hline $\begin{array}{l}241-A N-102 \\
(\mathrm{LAW})\end{array}$ & $\begin{array}{l}\text { No solid samples. No } \\
\text { analysis for many ICP and } \\
\text { IC analytes, most physical } \\
\text { properties, organics, and } \\
\text { radionuclides. }\end{array}$ & Liquid grabs & Liquid? & $\begin{array}{l}\text { May need solid } \\
\text { samples. }\end{array}$ \\
\hline $\begin{array}{l}241-\mathrm{AN}-103 \\
(\mathrm{LAW})\end{array}$ & $\begin{array}{l}\text { No analyses for most } \\
\text { physical properties, } \\
\text { organics, radionuclides, } \\
\text { some metals and others } \\
\text { (solids and liquids). }\end{array}$ & Liquid grabs & Liquid and solid & $\begin{array}{l}\text { May need solids } \\
\text { sample for } \\
\text { organics. }\end{array}$ \\
\hline $\begin{array}{l}\text { 241-AN-104 } \\
\text { (LAW) }\end{array}$ & $\begin{array}{l}\text { No analyses for most } \\
\text { physical properties, } \\
\text { organics, radionuclides, } \\
\text { some metals and others } \\
\text { (solids and liquids). }\end{array}$ & Liquid grabs & Liquid and solid & $\begin{array}{l}\text { May need solids } \\
\text { sample for } \\
\text { organics. }\end{array}$ \\
\hline \begin{tabular}{|l|}
$241-A N-105$ \\
(LAW)
\end{tabular} & $\begin{array}{l}\text { No analyses for most } \\
\text { physical properties, } \\
\text { organics, radionuclides, } \\
\text { some metals and others } \\
\text { (solids and liquids). }\end{array}$ & Liquid grabs & Liquid and solid & $\begin{array}{l}\text { May need solids } \\
\text { sample for } \\
\text { organics. }\end{array}$ \\
\hline $\begin{array}{l}\text { 241-AN-106 } \\
\text { (LAW) }\end{array}$ & $\begin{array}{l}\text { No solid samples. } \\
\text { Only have percent water, } \\
\text { gross alpha, DSC, total } \\
\text { organic carbon and SpG } \\
\text { (liquids). }\end{array}$ & $\begin{array}{l}\text { Push } \\
\text { liquid grabs }\end{array}$ & None & None \\
\hline $\begin{array}{l}\text { 241-AN-107 } \\
\text { (LAW) }\end{array}$ & $\begin{array}{l}\text { No solid samples. } \\
\text { Only have \% water, gross } \\
\text { alpha, DSC, total organic } \\
\text { carbon and SpG. }\end{array}$ & $\begin{array}{l}\text { Push } \\
\text { grabs }\end{array}$ & $\begin{array}{l}14 \text { vials content } \\
\text { unknown }\end{array}$ & None \\
\hline $\begin{array}{l}\text { 241-AW-101 } \\
\text { (LAW) }\end{array}$ & $\begin{array}{l}\text { No analyses for anions, } \\
\text { most physical properties, } \\
\text { organics, radionuclides, } \\
\text { some metals } \\
\text { (solids and liquids). }\end{array}$ & Liquid grabs & Liquid and solid & $\begin{array}{l}\text { May need solids } \\
\text { sample for } \\
\text { organics. }\end{array}$ \\
\hline
\end{tabular}


Table 6-1. Summary of Sample Data Not Available and Needs for Tank Privatization.

(3 sheets)

\begin{tabular}{|c|c|c|c|c|}
\hline (i: & 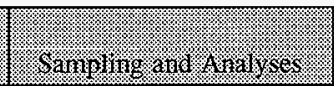 & $\begin{array}{l}\text { Sarvilings } \\
\text { Schedrated. }\end{array}$ & $\begin{array}{l}\text { Arenive } \\
\text { Samples: }\end{array}$ & 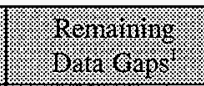 \\
\hline $\begin{array}{l}\text { 241-AY-102 } \\
\text { (HLW) }\end{array}$ & $\begin{array}{l}\text { No solid samples. } \\
\text { No analyses for many ICP } \\
\text { metals, organics, physical } \\
\text { properties except SpG, } \\
\text { many radionuclides } \\
\text { (solids and liquids). }\end{array}$ & Liquid grabs & None & $\begin{array}{l}\text { May need solid } \\
\text { samples. }\end{array}$ \\
\hline $\begin{array}{l}241-A Z-101 \\
\text { (LAW/ } \\
\text { HLW) }\end{array}$ & $\begin{array}{l}\text { No solid samples. } \\
\text { No analyses for many ICP } \\
\text { metals, organics, physical } \\
\text { properties except SpG, } \\
\text { many radionuclides } \\
\text { (solids and liquids). }\end{array}$ & $\begin{array}{l}\text { liquid and } \\
\text { solid grabs }\end{array}$ & None & None \\
\hline $\begin{array}{l}241-\text { AZ-102 } \\
\text { (LAW/ } \\
\text { HLW) }\end{array}$ & $\begin{array}{l}\text { No solid samples. } \\
\text { No analyses for many ICP } \\
\text { metals, organics, physical } \\
\text { properties except SpG, } \\
\text { many radionuclides } \\
\text { (solids and liquids). }\end{array}$ & $\begin{array}{l}\text { Liquid and } \\
\text { solid grabs }\end{array}$ & None & None \\
\hline $\begin{array}{l}\text { 241-SY-101 } \\
(\mathrm{LAW})\end{array}$ & $\begin{array}{l}\text { No analyses for some ICP } \\
\text { metals, organics, } \\
\text { radionuclides } \\
\text { (solids and liquids). }\end{array}$ & Nothing & None & $\begin{array}{l}\text { Need liquid and } \\
\text { solid samples, } \\
\text { may need grab } \\
\text { samples before } \\
\text { and after tank } \\
\text { transfers. }\end{array}$ \\
\hline $\begin{array}{l}\text { 241-SY-103 } \\
\text { (LAW) }\end{array}$ & $\begin{array}{l}\text { No analyses for many ICP } \\
\text { metals, physical properties, } \\
\text { organics, radionuclides } \\
\text { (solids and liquids). }\end{array}$ & Liquid grabs & Liquid and solid & $\begin{array}{l}\text { May need solid } \\
\text { sample for } \\
\text { organics. }\end{array}$ \\
\hline $\begin{array}{l}\text { 241-AP-102 } \\
\text { staging tank }\end{array}$ & NA & Grabs & $\mathrm{NA}$ & None \\
\hline $\begin{array}{l}\text { 241-AP-104 } \\
\text { staging tank }\end{array}$ & NA & Grabs. & $\mathrm{NA}$ & None \\
\hline $\begin{array}{l}\text { 241-AP-106 } \\
\text { feed tank }\end{array}$ & $\mathrm{NA}$ & Grabs & NA & None \\
\hline
\end{tabular}


HNF-2117 Rev. 0

Table 6-1. Summary of Sample Data Not Available and Needs for Tank Privatization. (3 sheets)

\begin{tabular}{|c|c|c|c|c|}
\hline 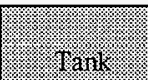 & 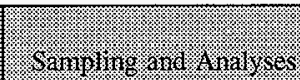 & $\begin{array}{l}\text { samplogy } \\
\text { schedined }\end{array}$ & 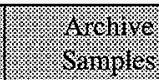 & 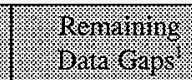 \\
\hline $\begin{array}{l}\text { 241-AP-108 } \\
\text { feed tank }\end{array}$ & $\mathrm{NA}$ & $\begin{array}{l}\text { October } 1997 \\
\text { grab }\end{array}$ & $\mathrm{NA}$ & $\begin{array}{l}\text { May need } \\
\text { additional grab } \\
\text { samples. }\end{array}$ \\
\hline
\end{tabular}

Note:

$\mathrm{NA}=$ not applicable

${ }^{14}$ None" means that based on current sample plans (see Table B-1) and/or archive samples, no additional sampling needs, beyond those scheduled, have been identified at this time. However, based on preliminary requirements, additional analyses are required for all tanks (see Tables A-2 and A-3). 
HNF-2117 Rev. 0

APPENDIX A

CHARACTERIZATION DATA REQUIREMENTS 
HNF-2117 Rev. 0

This page intentionally left blank.

A-2 
Table A-1. Low-Activity Waste and High-Level Waste Privatization Tank Data Requirements. (8 sheets)

\begin{tabular}{|c|c|c|c|c|c|c|c|c|c|c|c|}
\hline 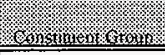 & 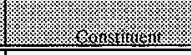 & 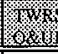 & 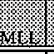 & 㙏18. & 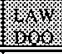 & 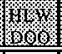 & 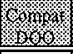 & 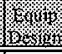 & 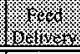 & 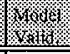 & 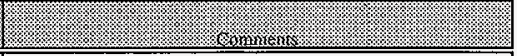 \\
\hline Anions & Chloride & $\mathrm{H}$ & & $\mathrm{L}$ & $\mathrm{L}$ & & $\mathrm{LH}$ & $\mathrm{LH}$ & & $\mathrm{LH}$ & \\
\hline Anions & Citrate & & & & $\mathrm{L}$ & & & & & & $\begin{array}{l}\text { Organics of interest for LAW DQO, Group II analytes are organic } \\
\text { acids, chelators, and semivolatiles. Some of them are listed } \\
\text { here. Will require IC, GC/MS \& perhaps other methods. }\end{array}$ \\
\hline Anions & Cyanide & $\mathrm{H}$ & & $\mathrm{H}$ & & & & & & $\mathrm{H}$ & \\
\hline Anions & EDTA & & & & & & & & & & \\
\hline Anions & Fluoride & $\mathrm{LH}$ & & L & $\mathrm{L}$ & $\mathrm{H}$ & & $\mathrm{LH}$ & & $\mathrm{LH}$ & \\
\hline Anions & Formate & & & & $\mathrm{L}$ & & & & & & \\
\hline Anions & HEDTA & & & & L & & $\cdot$ & & & & \\
\hline Anions & Nitrate & $\mathbf{L H}$ & & L & $\mathrm{L}$ & & $\mathrm{LH}$ & $\mathrm{LH}$ & & $\mathrm{LH}$ & \\
\hline Anions & Nitrite & LH & & L & L & & $\mathrm{LH}$ & $\mathrm{LH}$ & & $\mathrm{LH}$ & \\
\hline Anions & Phosphate & $\mathbf{L}$ & & $\mathrm{L}$ & $\mathrm{L}$ & & & $\mathrm{LH}$ & & $\mathrm{L}$ & \\
\hline Anions & Sulfate & L & & L & $\mathrm{L}$ & & & $\mathrm{LH}$ & & $\mathrm{L}$ & \\
\hline Inorganics. & Ammonia & $\mathrm{H}$ & & $\mathrm{H}$ & L & & & & LH & $\mathrm{H}$ & \\
\hline Inorganics & Carbonate & LH & & $L$ & & & & & & $\mathrm{LH}$ & \\
\hline Inorganics & Hexavalent chromium & & & & & & & & & & \\
\hline Inorganics & Hydroxide & $\mathbf{L}$ & & $\mathrm{L}$ & $\mathrm{L}$ & & $\mathrm{LH}$ & & & $\mathrm{L}$ & \\
\hline Inorganics & Total inorganic carbon & LH & & L & $\mathrm{L}$ & & & & & & \\
\hline Inorganics & Total organic carbon & LH & & $\mathrm{L}$ & $\mathrm{L}$ & & $\mathrm{LH}$ & & $\mathrm{LH}$ & $\mathrm{LH}$ & \\
\hline Metals & Aluminum & $\mathrm{LH}$ & & $\mathrm{L}$ & $\mathrm{L}$ & $\mathrm{H}$ & & $\mathrm{LH}$ & & $\mathrm{LH}$ & \\
\hline Metals & Antimony & $\mathrm{H}$ & & & $\mathrm{L}$ & & & $\mathrm{LH}$ & & $\mathrm{H}$ & \\
\hline Metals & Arsenic & $\mathrm{H}$ & & $\mathrm{H}$ & $\mathrm{L}$ & $\mathrm{H}$ & & $\mathrm{LH}$ & LH & $\mathrm{H}$ & \\
\hline Metals & Barium & $\mathrm{H}$ & & L & $\mathrm{L}$ & $\mathrm{H}$ & & LH & LH & LH & $\therefore$ \\
\hline Metals & Beryllium & $\mathrm{H}$ & & & $\mathrm{L}$ & & & $\mathrm{LH}$ & & $\mathrm{H}$ & \\
\hline Metals & Bismuth & $\mathbf{H}$ & & & $\mathrm{L}$ & $\mathrm{H}$ & & $\mathrm{LH}$ & & $\mathrm{LH}$ & \\
\hline Metals & Cadmium & LH & & $\mathrm{L}$ & $\mathrm{L}$ & $\mathrm{H}$ & & LH & LH & $\mathrm{LH}$ & \\
\hline Metals & Calcium & LH & & $\mathrm{L}$ & $\mathrm{L}$ & $\mathrm{H}$ & & LH & & $\mathrm{LH}$ & \\
\hline Metals & Cerium & $\mathrm{H}$ & & $\mathrm{H}$ & 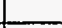 & $\mathrm{H}$ & & $\mathrm{LH}$ & & $\mathrm{H}$ & \\
\hline Metals & Cesium & $\mathrm{H}$ & & $\mathrm{H}$ & $\mathrm{L}$ & & & $\mathrm{LH}$ & & $\mathrm{H}$ & \\
\hline Metals & Chromium & LH & & $\mathrm{L}$ & $\mathrm{L}$ & $\mathrm{H}$ & & $\mathrm{LH}$ & $\mathrm{LH}$ & $\mathrm{LH}$ & \\
\hline Metals & Cobalt & $\mathrm{H}$ & & $\mathrm{H}$ & $\mathrm{L}$ & 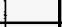 & & $\mathrm{LH}$ & & $\mathrm{H}$ & \\
\hline Metals & Copper & $\mathbf{H}$ & & $\mathrm{H}$ & $\mathrm{L}$ & $\mathrm{H}$ & & $\mathrm{LH}$ & & $\mathrm{H}$ & \\
\hline Metals & Dysprosium & $\mathrm{H}$ & & $\mathrm{H}$ & & & & $\mathrm{LH}$ & & $\mathrm{H}$ & \\
\hline Metals & Europium & $\mathbf{H}$ & & $\mathrm{H}$ & & & & LH & & $\mathrm{H}$ & \\
\hline Metals & Gadolinium & $\mathrm{H}$ & & & & & & $\mathrm{LH}$ & & $\mathrm{H}$ & \\
\hline Metals & Eron. & LH & & $\mathrm{L}$ & $\mathrm{L}$ & $\mathrm{H}$ & & $\mathrm{LH}$ & & $\mathrm{LH}$ & \\
\hline
\end{tabular}


Table A-1. Low-Activity Waste and High-Level Waste Privatization Tank Data Requirements. ( 8 sheets)

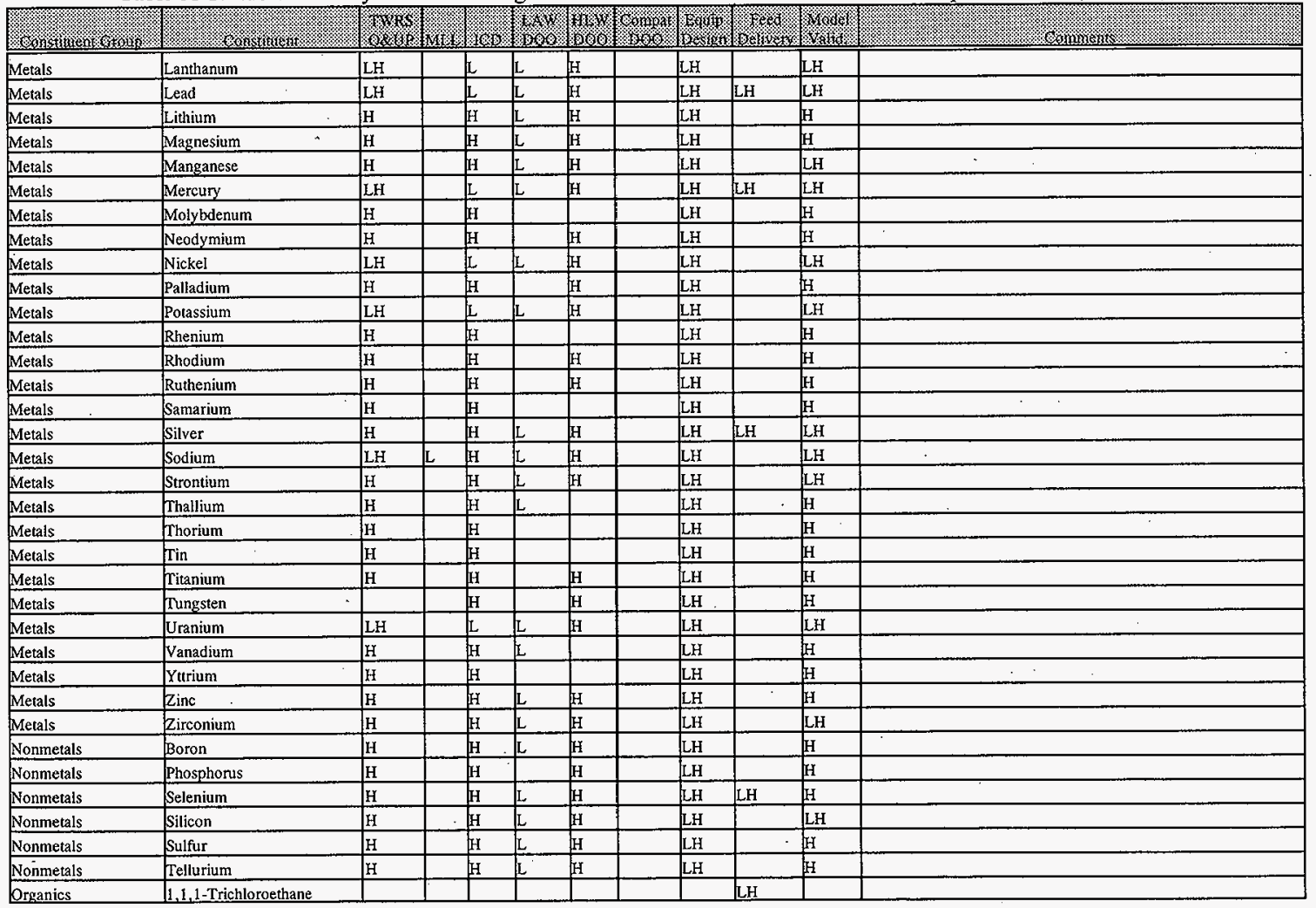


Table A-1. Low-Activity Waste and High-Level Waste Privatization Tank Data Requirements. (8 sheets)

\begin{tabular}{|c|c|c|c|c|c|c|c|c|c|c|c|}
\hline 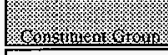 & 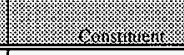 & Hxick & 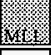 & 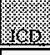 & $8 \%$ & 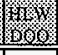 & wingos & ofsing & 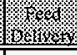 & 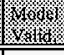 & W/. \\
\hline Organics & 1,2-Dichloroethane & & & & & & & & $\mathrm{LH}$ & & \\
\hline Organics & 2,4,5-Trichlorophenol & & & & & & & & $\mathrm{LH}$ & & \\
\hline Organics & 2,4-Dinitrotoluene & & & & & & & & $\mathrm{LH}$ & & \\
\hline Organics & 2-Butanone & & & & & & & & $\mathrm{LH}$ & & \\
\hline Organics & 2-Butoxyethanol & & & & & & & & $\mathrm{LH}$ & & \\
\hline Organics & 2-hexanone & & & & & & & & $\mathrm{LF}$ & & . \\
\hline Organics & 2-Pentanone & & & & & & & & $\mathrm{LH}$ & & \\
\hline Organics & 3-heptanone & & & & & & & & $\mathrm{LH}$ & & \\
\hline Organics & Acetate & & & & L & & & & & & \\
\hline Organics & Acetone & & & & & & & & $\mathrm{LH}$ & & \\
\hline Organics & Benzene & & & & & & & & $\mathrm{LH}$ & & 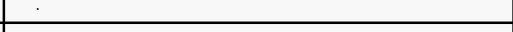 \\
\hline Organics & Carbon disulfide & & & & & & & & $\mathrm{LH}$ & & \\
\hline Organics & Carbon tetrachloride & & & & & & & & $\mathrm{LH}$ & & \\
\hline Organics & Chloroform & & & & & & & & $\mathrm{LH}$ & & \\
\hline Organics & Ethyl acetate & & & & & & & & $\mathrm{LH}$ & & \\
\hline Organics & Ethyl ether & & & & & & & & $\mathrm{LH}$ & & \\
\hline Organics & Glycolate & & & & $\mathrm{L}$ & . & & & & & \\
\hline Organics & Hexachlorobutadiene & & & & & & & & $\mathrm{LH}$ & & \\
\hline Organics & Hexachloroethane & & & & & & & & LH & & \\
\hline Organics & Methylenechloride & & & & & & & & $\mathrm{LH}$ & & - \\
\hline Organics & Naphthalene & & & & & & & & $\mathrm{LH}$ & & \\
\hline Organics & Nitrobenzene & & & & & & & & $\mathrm{LH}$ & & \\
\hline Organics & Nonane & & & & & & & & $\mathrm{LH}$ & & \\
\hline Organics & PCB & & & & & & & & $\mathrm{LH}$ & & \\
\hline Organics & Pyridine & & & & & & & & $\mathrm{LH}$ & & \\
\hline Organics & Tetrachloroethene & & & & & & & & $\mathrm{LH}$ & & \\
\hline Organics & Tetrahydrofuran & & & & & & & & $\mathrm{LH}$ & & \\
\hline Organics & Toluene & & & & & & & & LH & & \\
\hline Organics & Tributyl phosphate & & & & & & & & $\mathrm{LH}$ & & \\
\hline Organics & Vinyl chloride & & & & & & . & & $\mathrm{LH}$ & & \\
\hline Organics & Xylenes (total) & & & & & & & $\mathrm{LH}$ & $\mathrm{LH}$ & & \\
\hline Physical Properties & Density & $\mathbf{L H}$ & & & L & & & $\mathrm{LH}$ & & $\mathrm{LH}$ & \\
\hline Physical Properties & DSC (exotherm) & $L$ & & & & & $\mathrm{LH}$ & & & & . \\
\hline Physical Properties & Particle size & $\mathrm{H}$ & & & L & & & $\mathrm{LH}$ & & $H$ & $\begin{array}{l}\text { For LAW DQO, the composition of suspended or floating particles } \\
\text { should be determined by ICP, IC, total alpha and beta, and Sr-90, } \\
\text { particle size. }\end{array}$ \\
\hline
\end{tabular}


Table A-1. Low-Activity Waste and High-Level Waste Privatization Tank Data Requirements. (8 sheets)

\begin{tabular}{|c|c|c|c|c|c|c|c|c|c|c|c|}
\hline 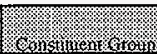 & 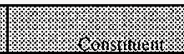 & Wx & 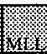 & 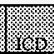 & 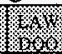 & $8 \%$ & (6) & 6x & 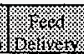 & 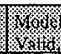 & 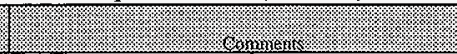 \\
\hline Physical Properties & Percent water & L & & & & & $\mathrm{LH}$ & & & & TGA Free water for compatability $\mathrm{DQO}$. \\
\hline Physical Properties & PH measurement & $\overline{\mathrm{LH}}$ & & $\mathrm{H}$ & & & $\mathrm{LH}$ & LH & & $\mathrm{LH}$ & \\
\hline Physical Properties & Settled solids & LH & & $\mathrm{H}$ & & & & & & $\mathrm{H}$ & \\
\hline Physical Properties & Shear strength & $\mathrm{H}$ & & $\mathrm{H}$ & & & & LH & & $\mathrm{H}$ & Of settled solids after 2 days \\
\hline Physical Properties & Solid density & $\mathrm{H}$ & & & & & & $\mathrm{LH}$ & & $\mathrm{H}$ & \\
\hline Physical Properties & Solid volume & $\mathbf{L}$ & & & & & & & & & \\
\hline Physical Properties & Specific gravity & $\mathrm{L}$ & & $\mathrm{H}$ & & & $\mathrm{LH}$ & $\mathrm{LH}$ & & & Slurry density \\
\hline Physical Properties & Temperature & & & & & & & LH & & & \\
\hline Physical Properties & Total dissolved solids & & & $\mathrm{H}$ & & & & & & & \\
\hline Physical Properties & Viscosity & & & $\mathrm{H}$ & $\mathrm{L}$ & & & $\mathrm{LH}$ & & $\mathrm{H}$ & Apparent Visc. $\mathrm{cP}$, for Equip Design DQO (supernatant). \\
\hline Physical Properties & \multicolumn{3}{|c|}{ Volume percent settled solids } & & $\mathrm{L}$ & & & & & & \\
\hline Physical Properties & Volume percent solids & $\mathrm{L}$ & & & & & & & & & \\
\hline Physical Properties & Weight percent oxides & $\mathrm{H}$ & & & & & & & & $\mathrm{H}$ & \\
\hline Physical Properties & Weight percent solids & LH & & & $\mathrm{L}$ & & & LH & & $\mathrm{H}$ & \\
\hline Physical Properties & Yield point & & & $\mathrm{H}$ & & & & & & $\mathrm{H}$ & Yield Stress dynes $/ \mathrm{cm}^{2}$ \\
\hline Radionuclides & Americium-241 & & & $\mathrm{H}$ & $\mathrm{L}$ & $\mathrm{H}$ & & & $\mathrm{LH}$ & $\mathrm{H}$ & \\
\hline Radionuclides & Americium-243 & & & $\mathrm{H}$ & $\mathrm{L}$ & $\mathrm{H}$ & & & & $\mathrm{H}$ & \\
\hline Radionuclides & Antimony-125 & & & $\mathrm{H}$ & & $\mathrm{H}$ & & & & & \\
\hline Radionuclides & Carbon-14 & & & $\mathrm{H}$ & $\mathrm{L}$ & $\mathrm{H}$ & & & & $\mathrm{H}$ & \\
\hline Radionuclides & Cerium-144 & & & $\mathrm{H}$ & & & & & $\mathrm{LH}$ & $\mathrm{H}$ & \\
\hline Radionuclides & \multicolumn{2}{|c|}{ Cerium/Praseodymium-144 } & & $\mathrm{H}$ & & & & & & $\mathrm{H}$ & \\
\hline Radionuclides & Cesium-134 & & & $\mathrm{H}$ & & & & & & $\mathrm{H}$ & \\
\hline Radionuclides & Cesium-137 & $\mathrm{L}$ & & $\mathrm{L}$ & L & $\mathrm{H}$ & & $\mathrm{LH}$ & $\mathrm{LH}$ & LH & \\
\hline Radionuclides & Cobalt -60 & & & $\mathrm{H}$ & $\mathrm{L}$ & $\mathrm{H}$ & & & $\mathrm{LH}$ & $\mathrm{H}$ & \\
\hline Radionuclides & Curium-242 & & & $\mathrm{H}$ & $\mathrm{L}$ & & & & & $\mathrm{H}$ & \\
\hline Radionuclides & Curium-243/244 & & & & $\mathrm{L}$ & $\mathrm{H}$ & & & & & \\
\hline Radionuclides & Curium-244 & & & $\mathrm{H}$ & & & & & & $\mathrm{H}$ & \\
\hline Radionuclides & Europium-152 & & & $\mathrm{H}$ & $\mathrm{L}$ & & & & LH & $\mathrm{H}$ & \\
\hline Radionuclides & Europium-154 & & & $\mathrm{H}$ & $\mathrm{L}$ & $\mathrm{H}$ & & & $\mathrm{LH}$ & $\mathrm{H}$ & \\
\hline Radionuclides & Europium-154/155 & & & . & $\mathrm{L}$ & & & & & & \\
\hline Radionuclides & Europium-155 & & & $\mathrm{H}$ & L & & & & & $\mathrm{H}$ & \\
\hline Radionuclides & Gross alpha & $\mathrm{L}$ & & $\mathrm{L}$ & $\mathrm{L}$ & & & & $\mathrm{EH}$ & $\mathrm{L}$ & or alpha from contributing radionuclides \\
\hline Radionuclides & Gross beta & & & 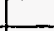 & L & & & & $\mathrm{LH}$ & & Pu-238,239,240, Am-241, maybe Am-242m and Cm-244 \\
\hline Radionuclides & Iodine-129 & & & $\mathrm{H}$ & L & $\mathrm{H}$ & & & EH & $\mathrm{H}$ & \\
\hline Radionuclides & Neptunium-237 & & & H & L & $\mathrm{H}$ & & & LH & $\mathrm{H}$ & \\
\hline
\end{tabular}


Table A-1. Low-Activity Waste and High-Level Waste Privatization Tank Data Requirements. (8 sheets)

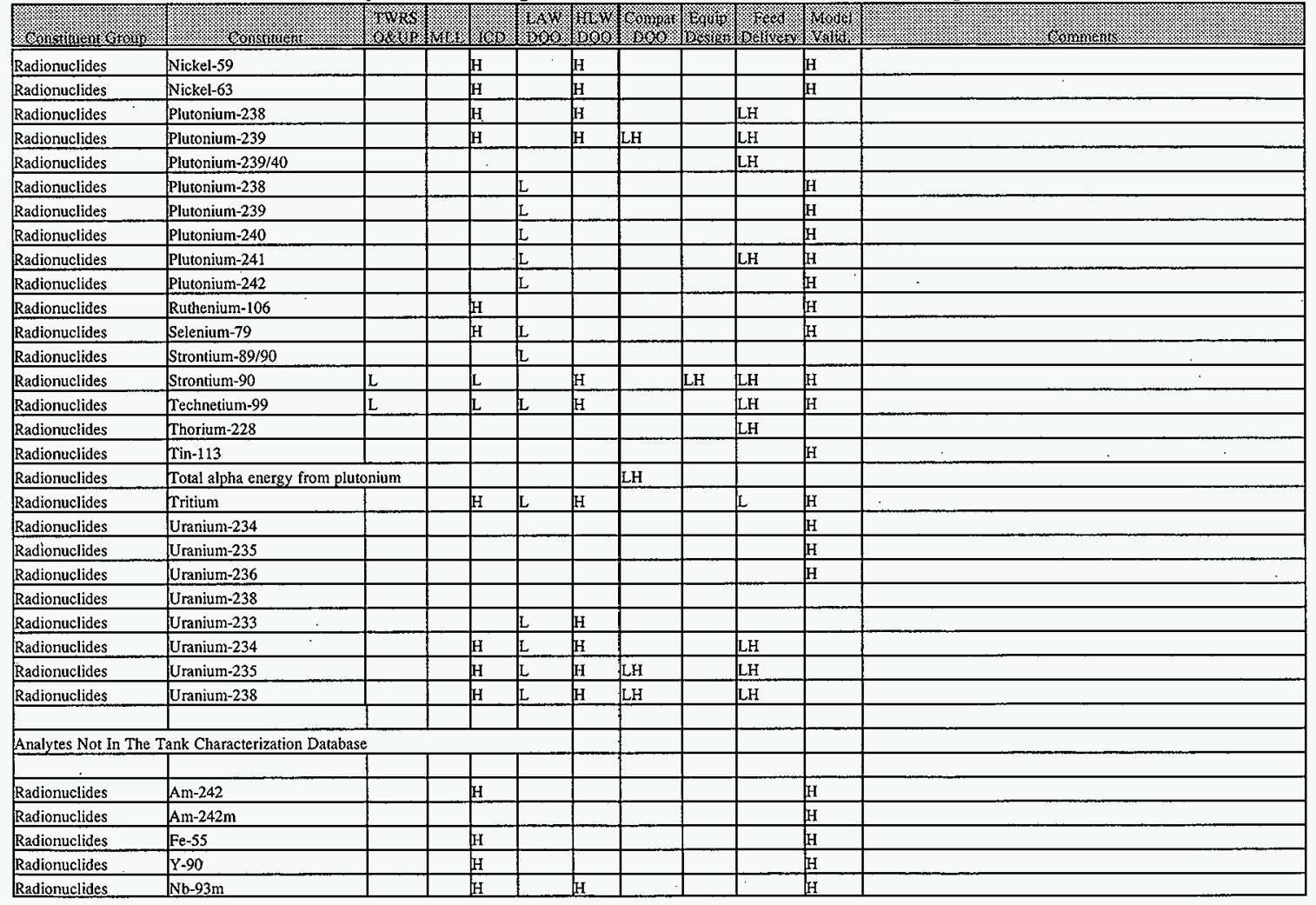


Table A-1. Low-Activity Waste and High-Level Waste Privatization Tank Data Requirements. (8 sheets)

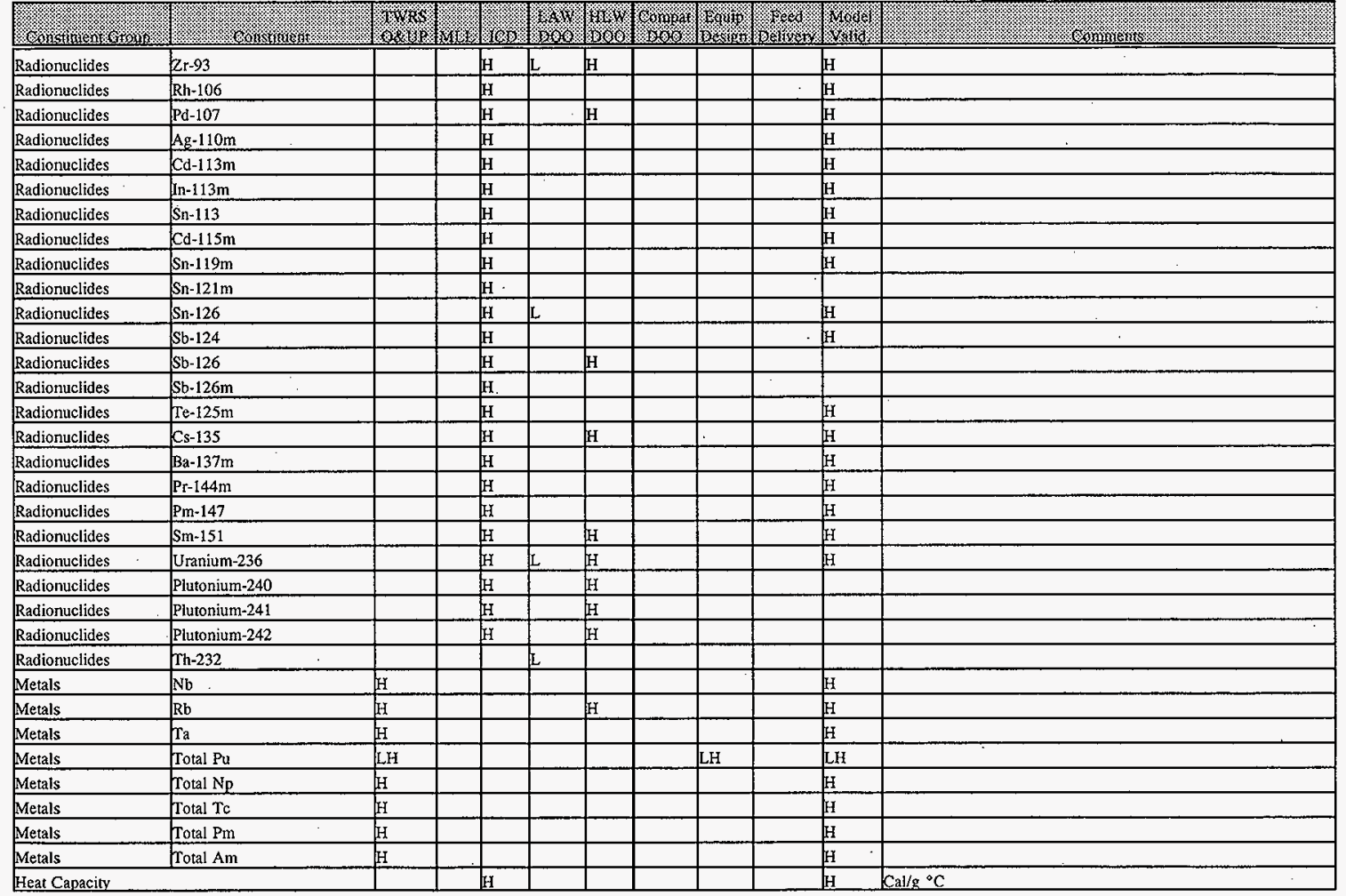


Table A-1. Low-Activity Waste and High-Level Waste Privatization Tank Data Requirements. (8 sheets)

\begin{tabular}{|c|c|c|c|c|c|c|c|c|c|c|c|}
\hline 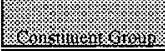 & 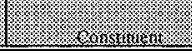 & 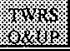 & 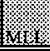 & 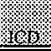 & 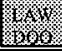 & 1) & 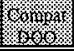 & 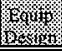 & Wxy. & W & 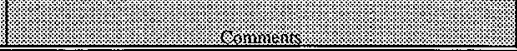 \\
\hline Metals & Rare Earths & & & & $\mathrm{L}$ & $\mathrm{H}$ & & & & & $\begin{array}{l}\text { Includes } \mathrm{Ce}, \mathrm{Pr}, \mathrm{Nd}, \mathrm{Pm}, \mathrm{Sm}, \mathrm{Eu}, \mathrm{Gd}, \mathrm{Tb}, \mathrm{Dy} \text {. Will require } \\
\text { ICP-MS. Indications are that a combined "less than" will be an } \\
\text { acceptable answer. }\end{array}$ \\
\hline Organics & Cresol total & & & & & & & & LH & & \\
\hline Organics & 1,1-dichloroethylene & & & & & & & & LH & & \\
\hline Organics & methyl ethyl ketone & & & & & & & & $\mathrm{LH}$ & & $\cdot$ \\
\hline Organics & trichloroethylene & & & & & & & & LH & & \\
\hline Organics & corrosivity & & & & & & & & $\mathrm{LH}$ & & \\
\hline Organics & ignitability & & & & & & & & LH & & \\
\hline Organics & reactivity & & & & & & & & $\mathrm{LH}$ & & \\
\hline Organics & methyl isobutyl ketone & & & & & & & & LH & & \\
\hline Organics & b-cresol & & & & & & & & $\mathrm{LH}$ & & \\
\hline Organics & p-cresol & & & & & & & & $\mathrm{LH}$ & & . \\
\hline Organics & m-cresol & & & & & & & & $\mathrm{LH}$ & & \\
\hline Organics & $\mathrm{Sb}-125$ & & & & & & & & $\mathrm{LH}$ & & \\
\hline Organics & $B i-214$ & & & & & & & & ILH & & \\
\hline Organics & acetonitrile & & & & & & & & $\mathrm{LH}$ & & \\
\hline Organics & $1-3$, butadiene & & & & & & & & LH & & \\
\hline Organics & butane & & & & & & & & $\mathrm{LH}$ & & \\
\hline Organics & In-butyl acetate & & & & & & & & $\mathrm{LH}$ & & \\
\hline Organics & n-butyl alcohol & & & & & & & & $\mathrm{LH}$ & & \\
\hline Organics & carbon monoxide & & & & & & . & & LH & & \\
\hline Organics & dipropyl ketone & & & & & & & & LH & & \\
\hline Organics & n-heptane & & & & & & & & $\mathrm{LH}$ & & \\
\hline Organics & n-hexane & & & & & & & & $\mathrm{LH}$ & & \\
\hline Organics & methyl n-amyl ketone & & & & & & & & $\mathrm{LH}$ & & \\
\hline Organics & nitric oxide & & & & & & & & $\mathrm{LH}$ & & \\
\hline Organics & mitrogen dioxide & & & & & & & & $\mathrm{LH}$ & & \\
\hline Organics & bctane & & & & & & & & $\mathrm{LH}$ & & \\
\hline Organics & bzone & & & & & & & & $\mathrm{LH}$ & & \\
\hline Organics & particulates PM-10 & & & & & & & & $\mathrm{LH}$ & & \\
\hline Organics & pentane & & & & & & & & $\mathrm{LH}$ & & . \\
\hline Organics & sulfur dioxide & & & & & & & & $\mathrm{LH}$ & & \\
\hline Organics & trichlorofluoromethane & & & & & & & & $\mathrm{LH}$ & & \\
\hline Organics & vinylidene chloride & & & & & & & & $\mathrm{LH}$ & & \\
\hline Metals & Praseodymium & & & & & $\mathrm{H}$ & & & & & \\
\hline
\end{tabular}




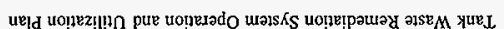

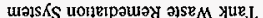
ue[d אi!!!

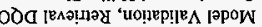

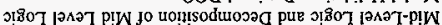

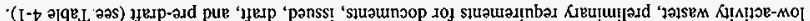

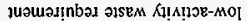

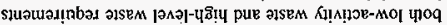

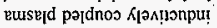

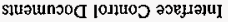

$$
\text { Kपded }
$$

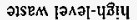

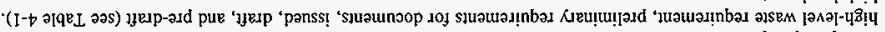

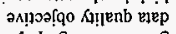

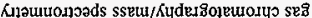

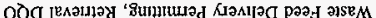
oOd jexa!nay 'uAisad puaudinbg

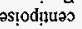
OOC Al!lqqueduro มวุวu!̣นว วגenbs

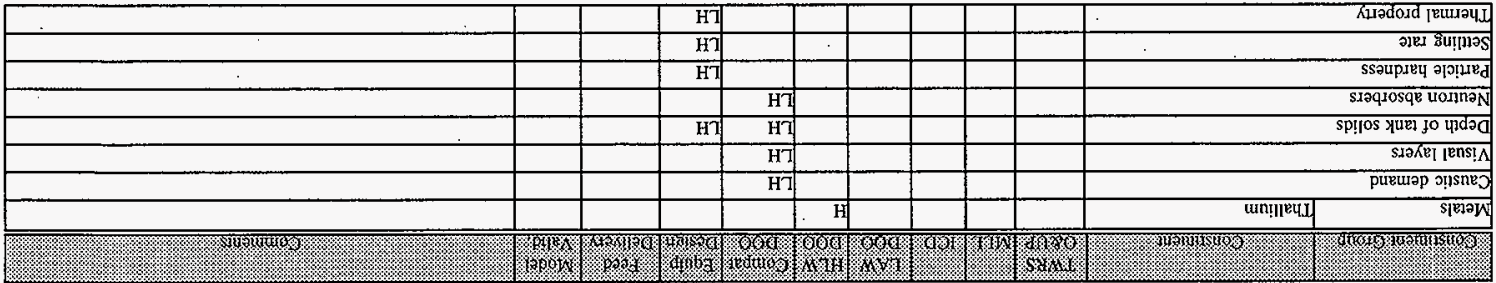


Table A-2. Available and Needed Data for Required Low-Activity Waste Analyses. ${ }^{1,2}$ (41 sheets)

\begin{tabular}{|c|c|c|c|c|c|c|}
\hline 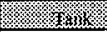 & 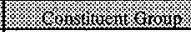 & \% & 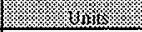 & Y. & 3rix & 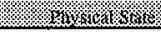 \\
\hline 241-AN-102 & Anions & Chloride & $\mu \mathrm{g} / \mathrm{mL}$ & Supernatant & Grab sample & Liquid \\
\hline 241-AN-102 & Anions & Citrate & & & & \\
\hline 241-AN-102 & Anions & Fluoride & $\mu \mathrm{g} / \mathrm{mL}$ & Supernatant & Grab sample & Liquid \\
\hline 241-AN-102 & Anions & Formate & & & & \\
\hline 241-AN-102 & Anions & HEDTA & & & & \\
\hline 241-AN-102 & Anions & Nitrate & $\mu \mathrm{g} / \mathrm{mL}$ & Supernatant & Grab sample & Liquid \\
\hline 241-AN-102 & Anions & Nitrite & $\mu \mathrm{g} / \mathrm{mL}$ & Supernatant & Grab sample & Liquid \\
\hline 241-AN-102 & Anions & Phosphate & $\mu \mathrm{g} / \mathrm{mL}$ & Supernatant & Grab sample & Liquid \\
\hline 241-AN-102 & Anions & Sulfate & $\mu \mathrm{g} / \mathrm{mL}$ & Supematant & Grab sample & Liquid \\
\hline 241-AN-102 & Inorganics & Ammonia & & & & \\
\hline 241-AN-102 & Inorganics & Carbonate & & & & \\
\hline 241-AN-102 & Inorganics & Hydroxide & $\mu \mathrm{g} / \mathrm{mL}$ & Supernatant & Grab sample & Liquid \\
\hline 241-AN-102 & Inorganics & Total inorganic carbon & $\mu \mathrm{g} / \mathrm{mL}$ & Supernatant & Grab sample & Liquid \\
\hline $241-\mathrm{AN}-102$ & Inorganics & Total organic carbon & $\mu \mathrm{g} / \mathrm{mL}$ & Supernatant & Grab sample & Liquid \\
\hline $241-A N-102$ & Metals & Aluminum & $\mu \mathrm{g} / \mathrm{mL}$ & Supernatant & Grab sample & Liquid \\
\hline 241-AN-102 & Metals & Antimony & & & & \\
\hline $241-\mathrm{AN}-102$ & Metals & Arsenic & & & & \\
\hline $241-\mathrm{AN}-102$ & Metals & Barium & & & & \\
\hline 241-AN-102 & Metals & Beryllium & & & & \\
\hline $241-\mathrm{AN}-102$ & Metals & Bismuth & & & & \\
\hline $241-\mathrm{AN}-102$ & Metals & Cadmium & & & & \\
\hline $241 \cdot \mathrm{AN}-102$ & Metals & Calcium & & & & \\
\hline 241-AN-102 & Metals & Cesium & & & & \\
\hline $241 \cdot \mathrm{AN}-102$ & Metals & Cobalt & & & & \\
\hline $241-\mathrm{AN}-102$ & Metals & Copper & & & & \\
\hline 241-AN-102 & Metals & Iron & $\mu \mathrm{g} / \mathrm{mL}$ & Supernatant & Grab sample & Liquid \\
\hline $241-\mathrm{AN}-102$ & Metals & Lanthanum & & & & \\
\hline $241-\mathrm{AN}-102$ & Metals & Lead & & & & \\
\hline 241-AN-102 & Metals & Lithium & & & & \\
\hline 241-AN-102 & Metals & Magnesium & & & & \\
\hline 241-AN-102 & Metals & Manganese & $\mu \mathrm{g} / \mathrm{mL}$ & Supernatant & Grab sample & Liquid \\
\hline $241-\mathrm{AN}-102$ & Metals & Mercury & & & & \\
\hline 241-AN-102 & Metals & Nickel & $\mu \mathrm{g} / \mathrm{mL}$ & Supernatant & Grab sample & Liquid \\
\hline 241-AN-102 & Metals & Potassium & & & & \\
\hline 241-AN-102 & Metals & Silver & . & & & . \\
\hline 241-AN-102 & Metals & Sodium & $\mu \mathrm{g} / \mathrm{mL}$ & Supernatant & Grab sample & Liquid \\
\hline
\end{tabular}


Table A-2. Available and Needed Data for Required Low-Activity Waste Analyses. ${ }^{1,2}$ (41 sheets)

\begin{tabular}{|c|c|c|c|c|c|c|}
\hline 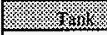 & 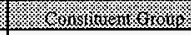 & XY) & 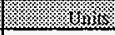 & 保 & 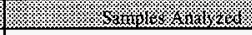 & 箖籍 \\
\hline 241-AN-102 & Metals & Strontium & & & & \\
\hline 241-AN-102 & Metals & Thallium & & & & \\
\hline 241-AN-102 & Metals & Uranium & $\mu \mathrm{g} / \mathrm{mL}$ & Supernatant & Grab sample & Liquid \\
\hline $241-\mathrm{AN}-102$ & Metals & Vanadium & & & & \\
\hline 241-AN-102 & Metals & Zinc & & & & \\
\hline 241-AN-102 & Metals & Zirconium & & & & \\
\hline 241-AN-102 & Nonmetals & Boron & & 1. & & \\
\hline $241-\mathrm{AN}-102$ & Nonmetals & Selenium & & & & \\
\hline $241-\mathrm{AN}-102$ & Nonmetals & Silicon & $\mu \mathrm{g} / \mathrm{mL}$ & Supernatant & Grab sample & Liquid \\
\hline 241-AN-102 & Nonmetals & Sulfur & & & & \\
\hline 241-AN-102 & Nonmetals & Tellurium & & & & \\
\hline 241-AN-102 & Organics & 1,1,1-Trichloroethane & & & & \\
\hline 241-AN-102 & Organics & 1,2-Dichloroethane & & & & \\
\hline 241-AN-102 & Organics. & 2,4,5-Trichlorophenol & & & & \\
\hline 241-AN-102 & Organics & 2,4-Dinitrotoluene & & & & \\
\hline 241-AN-102. & Organics & 2-Butanone & & & & . \\
\hline 241-AN-102 & Organics, & 2-Butoxyethanol & & & & \\
\hline 241-AN-102 & Organics & 2-Hexanone & & & & \\
\hline 241-AN-102 & Organics & 2-Pentanone & & & & \\
\hline 241-AN-102 & Organics & 3-Heptanone & & & & \\
\hline 241-AN-102 & Organics & Acetate & & & & \\
\hline 241-AN-102 & Organics & Acetone & & & & \\
\hline 241-AN-102 & Organics & Benzene & & & & \\
\hline 241-AN-102 & Organics & Carbon disulfide & & & & \\
\hline 241-AN-102 & Organics & Carbon tetrachloride & & & & \\
\hline 241-AN-102 & Organics & Chloroform & & & & \\
\hline 241-AN-102 & Organics & Ethyl acetate & & & & \\
\hline 241-AN-102 & Organics & Ethyl ether & & & & \\
\hline $241-A N-102$ & Organics & Glycolate & & & & \\
\hline 241-AN-102 & Organics & Hexachlorobutadiene & & & $\therefore$ & \\
\hline 241-AN-102 & Organics & Methylenechloride & & & & \\
\hline 241-AN-102 & Organics & Naphthalene & & & & \\
\hline $241-\mathrm{AN}-102$ & Organics & Nitrobenzene & & & & \\
\hline 241-AN-102 & Organics & Nonane & & & & \\
\hline $241-A N-102$ & Organics & PCB & & & & \\
\hline 241-AN-102 & Organics & Pyridine & & & & \\
\hline
\end{tabular}


Table A-2. Available and Needed Data for Required Low-Activity Waste Analyses. ${ }^{1,2}$ (41 sheets)

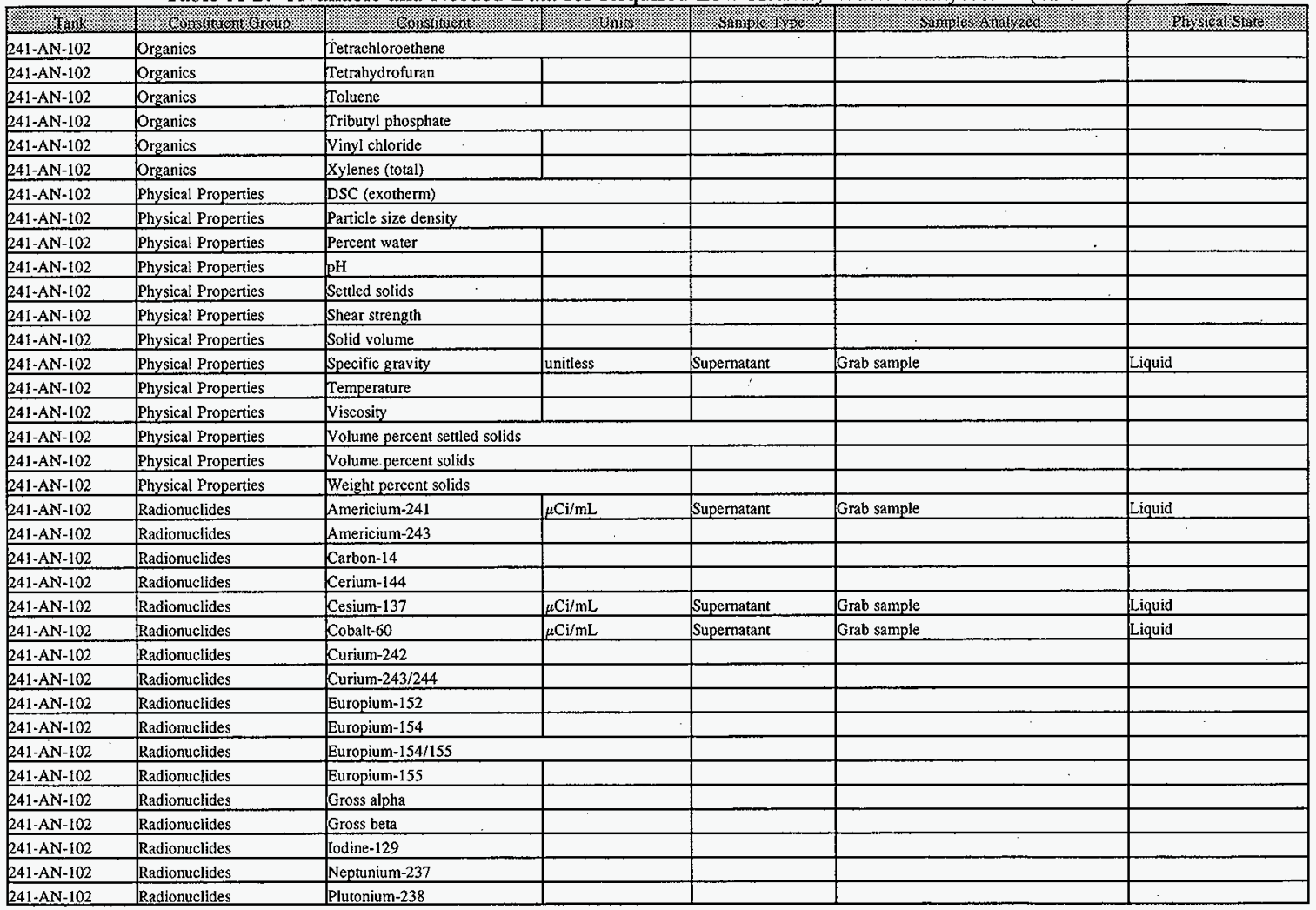




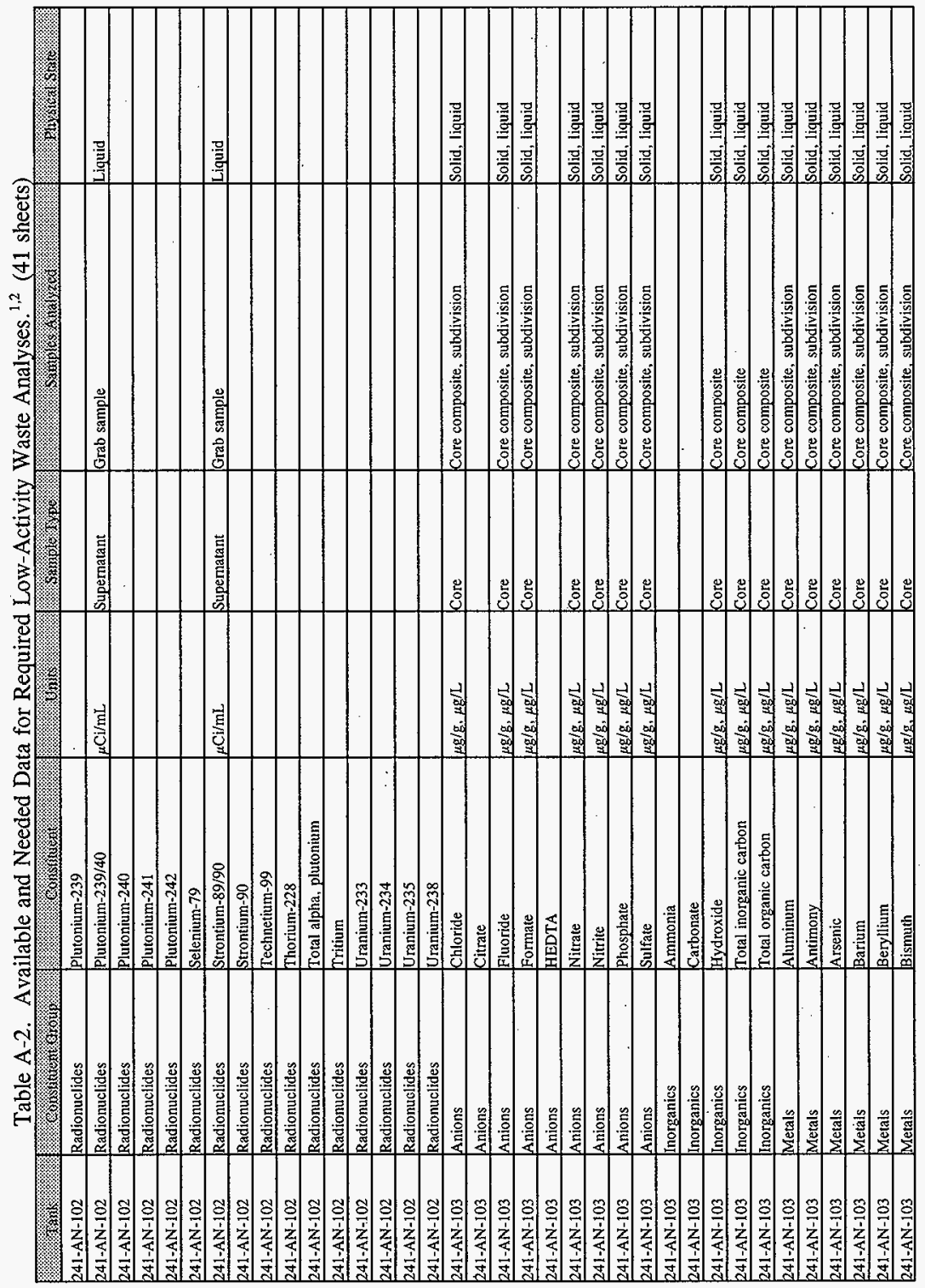


Table A-2. Available and Needed Data for Required Low-Activity Waste Analyses. ${ }^{1,2}$ (41 sheets)

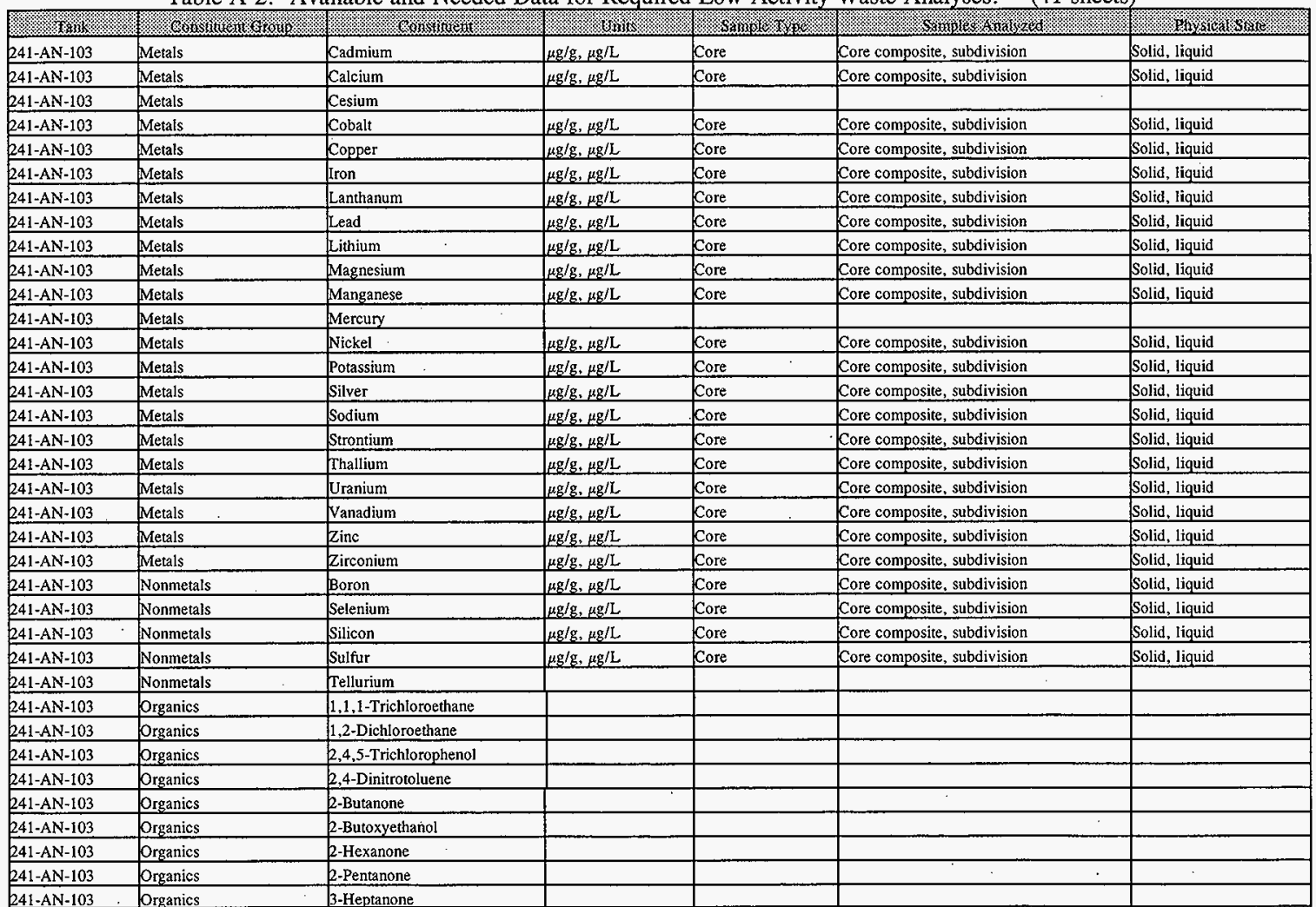


Table A-2. Available and Needed Data for Required Low-Activity Waste Analyses. ${ }^{1,2}$ (41 sheets)

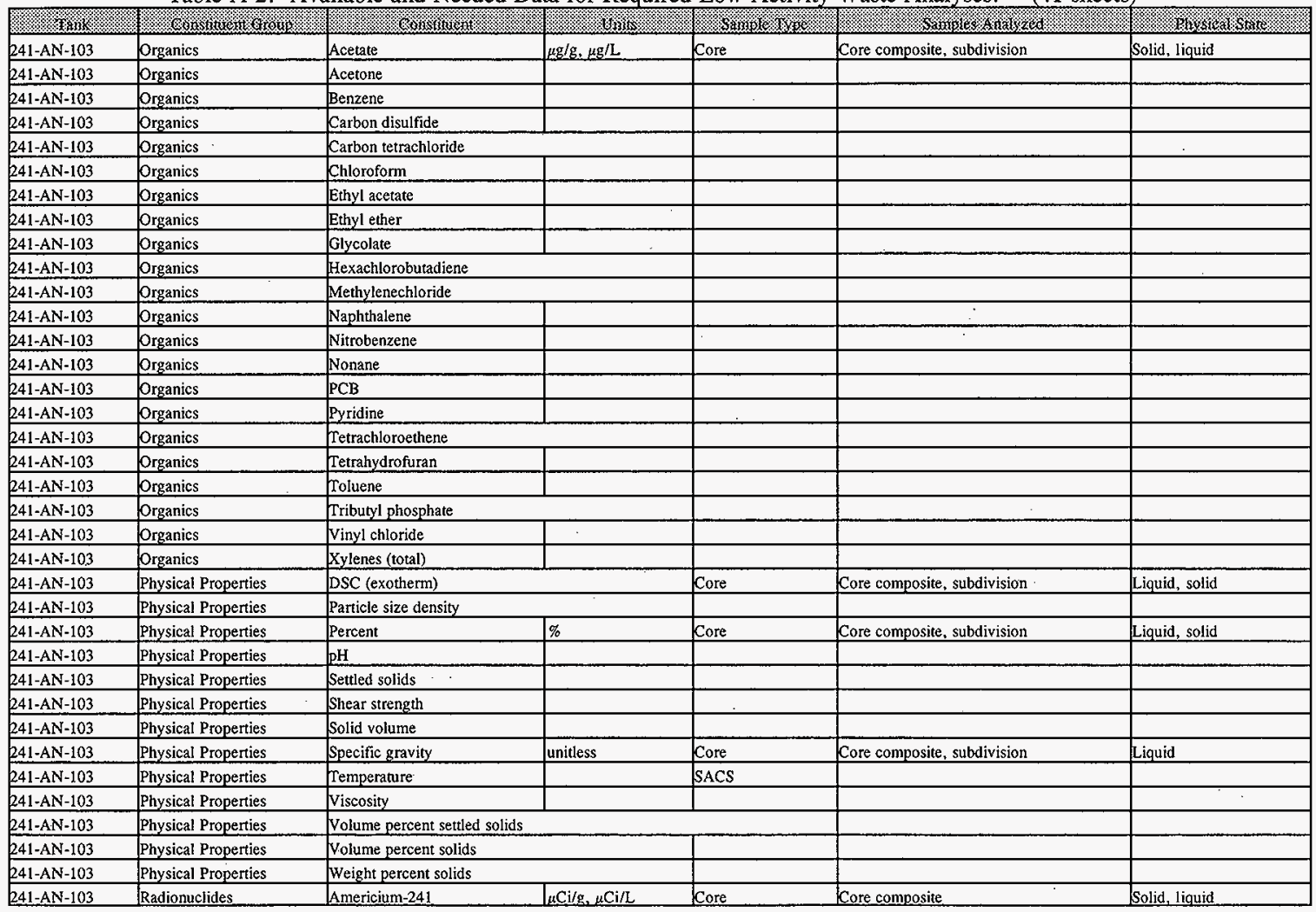


Table A-2. Available and Needed Data for Required Low-Activity Waste Analyses. ${ }^{1,2}$ (41 sheets)

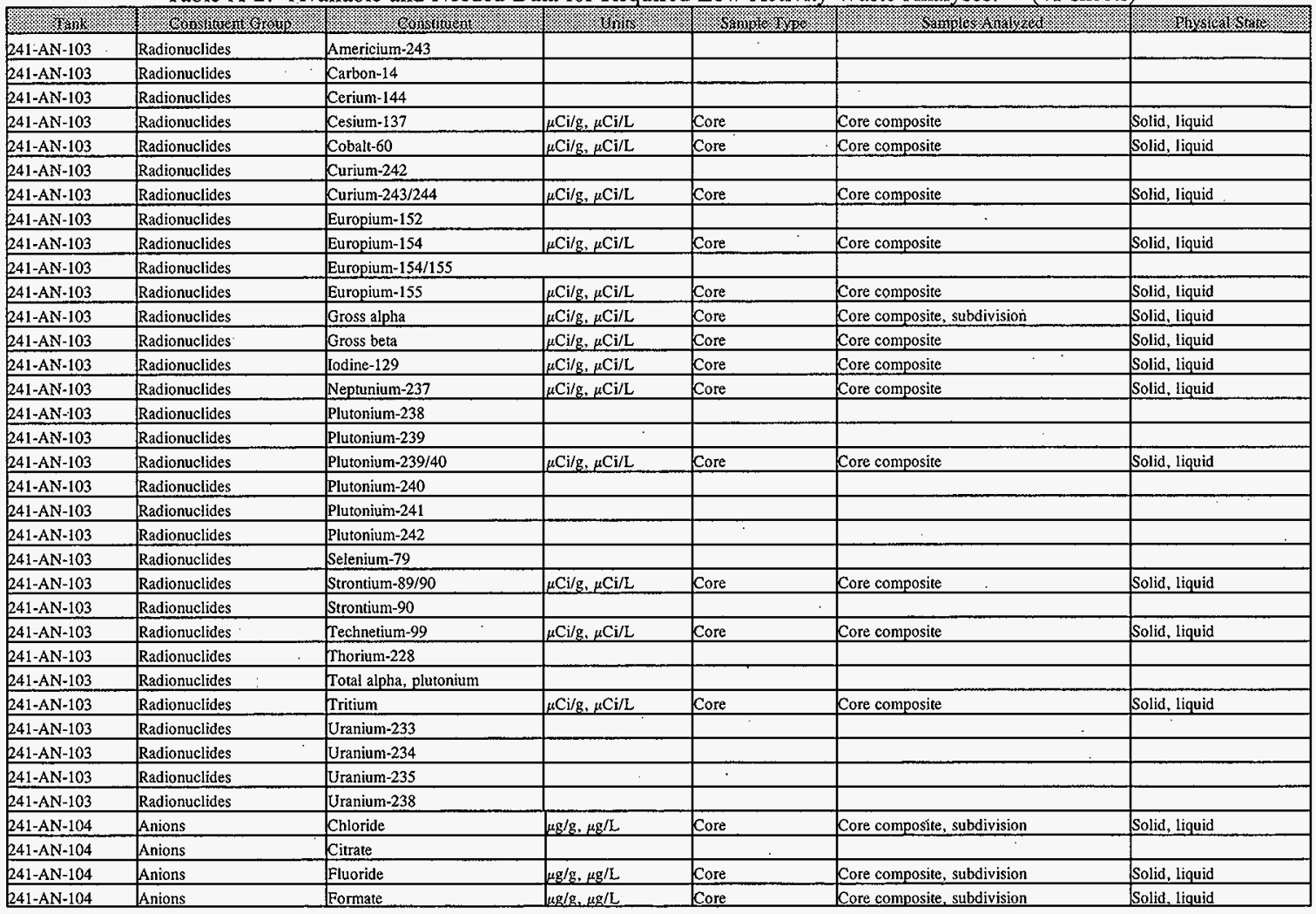


Table A-2. Available and Needed Data for Required Low-Activity Waste Analyses. ${ }^{1,2}$ (41 sheets)

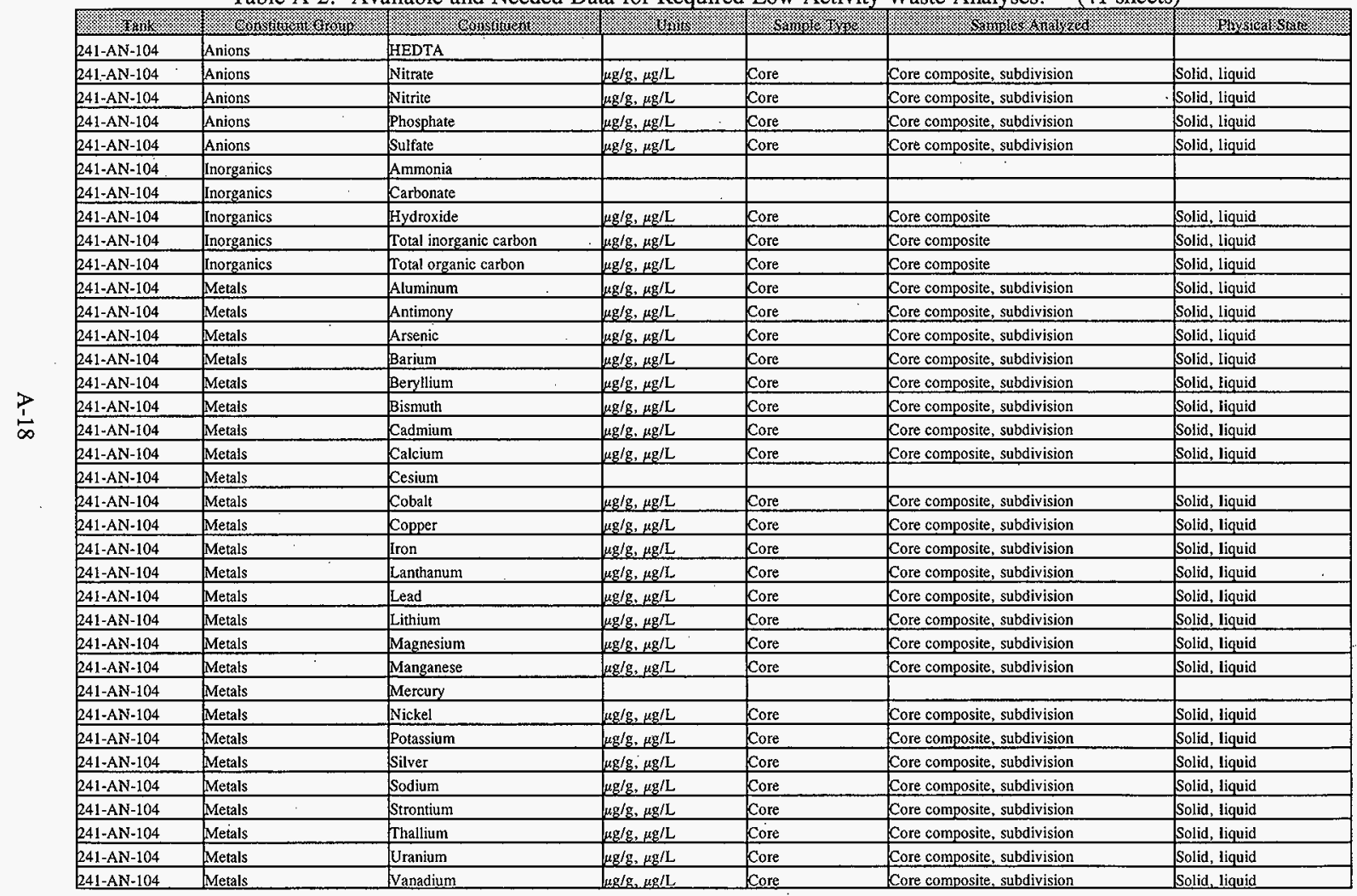


Table A-2. Available and Needed Data for Required Low-Activity Waste Analyses ${ }^{1,2}$ (41 sheets)

\begin{tabular}{|c|c|c|c|c|c|c|}
\hline 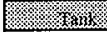 & W. & 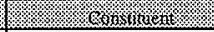 & 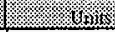 & 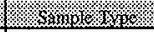 & 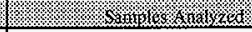 & 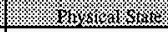 \\
\hline $241-\mathrm{AN}-104$ & Metals & Zinc & $\mu \mathrm{g} / \mathrm{g}, \mu \mathrm{g} / \mathrm{L}$ & Core & Core composite, subdivision & Solid, liquid \\
\hline 241-AN-104 & Metals & Zirconium & $\mu \mathrm{g} / \mathrm{g}, \mu \mathrm{g} / \mathrm{L}$ & Core & Core composite, subdivision & Solid, liquid \\
\hline 241-AN-104 & Nonmetals & Boron & $\mu \mathrm{g} / \mathrm{g}, \mu \mathrm{g} / \mathrm{L}$ & Core & Core composite, subdivision & Solid, liquid \\
\hline 241-AN-104 & Nonmetals & Selenium & $\mu \mathrm{g} / \mathrm{g}, \mu \mathrm{g} / \mathrm{L}$ & Core & Core composite, subdivision & Solid, liquid \\
\hline $241-\mathrm{AN}-104$ & Nonmetals & Silicon & $\mu \mathrm{g} / \mathrm{g} . \mu \mathrm{g} / \mathrm{L}$ & Core & Core composite, subdivision & Solid, liquid \\
\hline 241-AN-104 & Nonmetals & Sulfur & $\mu \mathrm{g} / \mathrm{g}, \mu \mathrm{g} / \mathrm{L}$ & Core & Core composite, subdivision & Solid, liquid \\
\hline 241-AN-104 & Nonmetals & Tellurium & & & & \\
\hline 241-AN-104 & Organics & 1,1,1-Trichloroethane & & & & \\
\hline 241-AN-104 & Organics & 1,2-Dichloroethane & & & & \\
\hline $241-\mathrm{AN}-104$ & Organics & 2,4,5-Trichlorophenol & & & & \\
\hline 241-AN-104 & Organics & 2,4-Dinitrotoluene & & & & \\
\hline 241-AN-104 & Organics & 2-Butanone & & & & \\
\hline 241-AN-104 & Organics & 2-Butoxyethanol & & & & \\
\hline 241-AN-104 & Organics & 2-Hexanone & & & & \\
\hline 241-AN-104 & Organics & 2-Pentanone & & & & \\
\hline 241-AN-104 & Organics & 3-Heptanone & & & & \\
\hline 241-AN-104 & Organics & Acetate & $\mu \mathrm{g} / \mathrm{g}, \mu \mathrm{g} / \mathrm{L}$ & Core & Core composite, subdivision & Solid, liquid \\
\hline 241-AN-104 & Organics & Acetone & & & & \\
\hline 241-AN-104 & Organics & Benzene & & & & \\
\hline 241-AN-104 & Organics & Carbon disulfide & & & & - \\
\hline 241-AN-104 & Organics & Carbon tetrachloride & & & & \\
\hline 241-AN-104 & Organics & Chloroethane & & & & \\
\hline $241-\mathrm{AN}-104$ & Organics & Chloroform & & & & \\
\hline 241-AN-104 & Organics & Ethane & & & & \\
\hline $241-\mathrm{AN}-104$ & Organics & Ethyl acetate & & & & \\
\hline 241-AN-104 & Organics & Ethyl ether & & & & \\
\hline 241-AN-104 & Organics & Glycolate & & & & \\
\hline 241-AN-104 & Organics & Hexachlorobutadiene & & & & \\
\hline 241-AN-104 & Organics & Hexachloroethane & & & & \\
\hline $241-\mathrm{AN}-104$ & Organics & Methylenechloride & & & & \\
\hline 241-AN-104 & Organics & Naphthalene & & & & \\
\hline 241-AN-104 & Organics & Nitrobenzene & & & & \\
\hline 241-AN-104 & Organics & Nonane & & & & \\
\hline 241-AN-104 & Organics & $\mathrm{PCB}$ & & & & \\
\hline 241-AN-104 & Organics & Phenol & & & & \\
\hline 241-AN-104 & Organics & Pyridine & & & & \\
\hline
\end{tabular}


Table A-2. Available and Needed Data for Required Low-Activity Waste Analyses. ${ }^{1,2}$ (41 sheets)

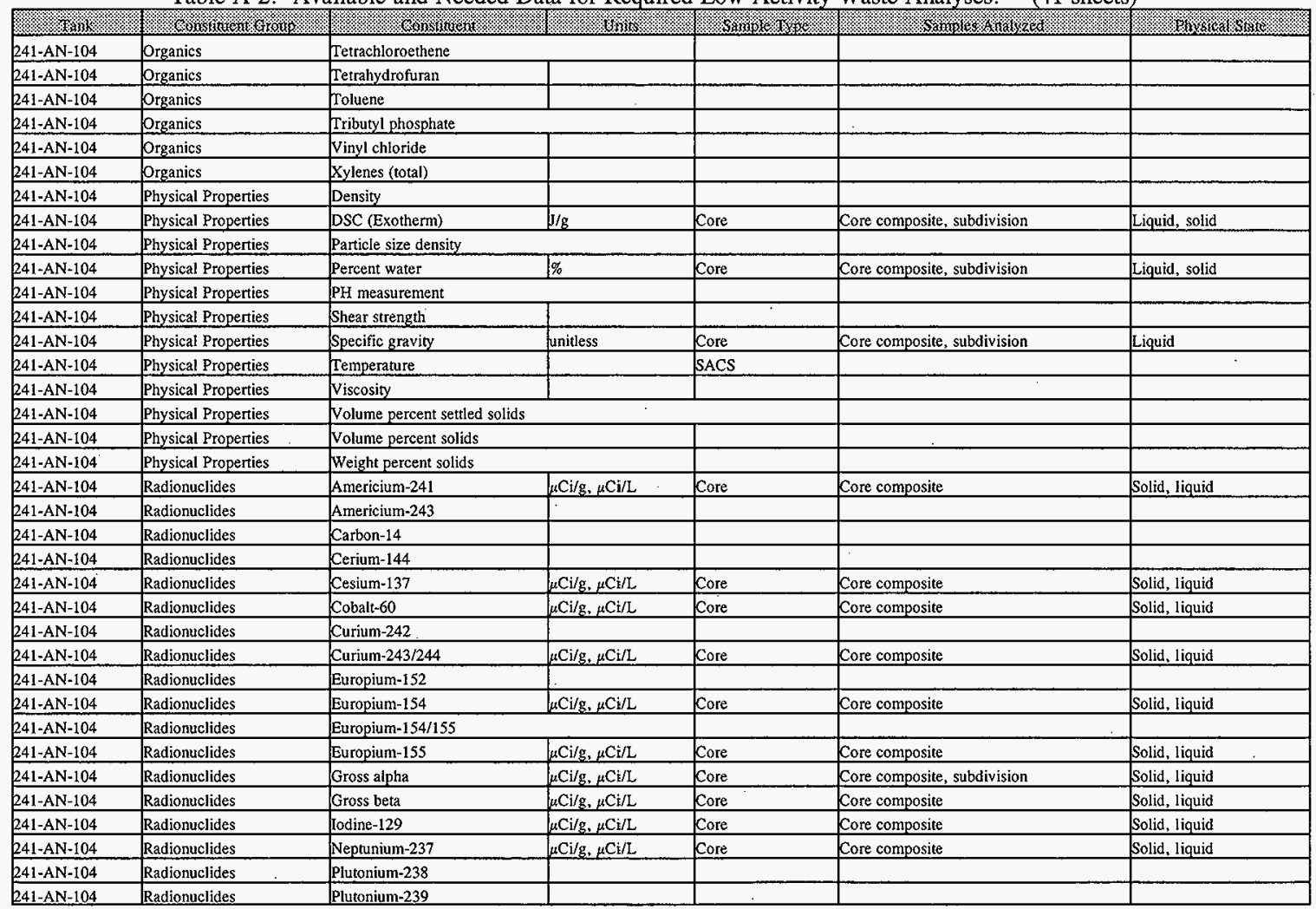


Table A-2. Available and Needed Data for Required Low-Activity Waste Analyses. ${ }^{1,2}$ (41 sheets)

\begin{tabular}{|c|c|c|c|c|c|c|}
\hline 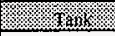 & 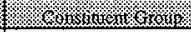 & 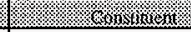 & 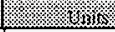 & 湆 & W/ & 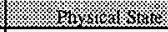 \\
\hline 241-AN-104 & Radionuclides & Plutonium-239/40 & $\mu \mathrm{Ci} / \mathrm{g}, \mu \mathrm{Ci} / \mathrm{L}$ & Core & Core composite & Solid, liquid \\
\hline $241-\mathrm{AN}-104$ & Radionuclides & Plutonium-240 & & & & \\
\hline $241-\mathrm{AN}-104$ & Radionuclides & Plutonium-241 & & & & \\
\hline 241-AN-104 & Radionuclides & Plutonium-242 & & . & & \\
\hline $241-\mathrm{AN}-104$ & Radionuclides & Selenium-79 & & & & \\
\hline 241-AN-104 & Radionuclides & Strontium-89/90 & $\mu \mathrm{Ci} / \mathrm{g}, \mu \mathrm{Ci} / \mathrm{L}$ & Core & Core composite & Solid, liquid \\
\hline 241-AN-104 & Radionuclides & Strontium-90 & & & & \\
\hline 241-AN-104 & Radionuclides & Technetium-99 & $\mu \mathrm{Ci} / \mathrm{g}, \mu \mathrm{Ci} / \mathrm{L}$ & Core & Core composite & Solid, liquid \\
\hline 241-AN-104 & Radionuclides & Thorium-228 & & & & \\
\hline 241-AN-104 & Radionuclides & Total alpha, plutonium & & & & \\
\hline 241-AN-104 & Radionuclides & Tritium & $\mu \mathrm{Ci} / \mathrm{g}, \mu \mathrm{Ci} / \mathrm{L}$ & Core & Core composite & Solid, liquid \\
\hline 241-AN-104 & Radionuclides & Uranium-233 & & & & \\
\hline 241-AN-104 & Radionuclides & Uranium-234 & & & & \\
\hline 241-AN-104 & Radionuclides & Uranium-235 & & & & \\
\hline 241-AN-104 & Radionuclides & Uranium-238 & & & & \\
\hline $241-\mathrm{AN}-105$ & Anions & Chloride & $\mu \mathrm{g} / \mathrm{g}, \mu \mathrm{g} / \mathrm{L}$ & Core & Core composite, subdivision & Solid, liquid \\
\hline 241-AN-105 & Anions & Citrate & & & & \\
\hline $241-\mathrm{AN}-105$ & Anions & Fluoride & $\mu g / g . \mu g / L$ & Core & Core composite, subdivision & Solid, liquid \\
\hline $241-\mathrm{AN}-105$ & Anions & Formate & $\mu \mathrm{g} / \mathrm{g}, \mu \mathrm{g} / \mathrm{L}$ & Core & Core composite, subdivision & Solid, liquid \\
\hline $241-\mathrm{AN}-105$ & Anions & HEDTA & & & & \\
\hline $241-\mathrm{AN}-105$ & Anions & Nitrate & $\mu \mathrm{g} / \mathrm{g}, \mu \mathrm{g} / \mathrm{L}$ & Core & Core composite, subdivision & Solid, Liquid \\
\hline 241-AN-105 & Anions & Nitrite & $\mu \mathrm{g} / \mathrm{g}, \mu \mathrm{g} / \mathrm{L}$ & Core & Core composite, subdivision & Solid, liquid \\
\hline $241-\mathrm{AN}-105$ & Anions & Phosphate & $\mu \mathrm{g} / \mathrm{g}, \mu \mathrm{g} / \mathrm{L}$ & Core & Core composite, subdivision & Solid, liquid \\
\hline 241-AN-105 & Anions & Sulfate & $\mu \mathrm{g} / \mathrm{g}, \mu \mathrm{g} / \mathrm{L}$ & Core & Core composite, subdivision & Solid, liquid \\
\hline 241-AN-105 & Inorganics & Ammonia & & & & \\
\hline 241-AN-105 & Inorganics & Carbonate & & & & \\
\hline $241-\mathrm{AN}-105$ & Inorganics & Hydroxide & $\mu \mathrm{g} / \mathrm{g}, \mu \mathrm{g} / \mathrm{L}$ & Core & Core composite & Solid, liquid \\
\hline 241-AN-105 & Inorganics & Total inorganic carbon & $\mu \mathrm{g} / \mathrm{g}, \mu \mathrm{g} / \mathrm{L}$ & Core & Core composite & Solid, liquid \\
\hline 241-AN-105 & Inorganics & Total organic carbon & $\mu \mathrm{g} / \mathrm{g} . \mu \mathrm{g} / \mathrm{L}$ & Core & Core composite & Solid, liquid \\
\hline 241-AN-105 & Metals & Aluminum & $\mu \mathrm{g} / \mathrm{g}, \mu \mathrm{g} / \mathrm{L}$ & Core & Core composite, subdivision & Solid, liquid \\
\hline 241-AN-105 & Metals & Antimony & $\mu \mathrm{g} / \mathrm{g}, \mu \mathrm{g} / \mathrm{L}$ & Core & Core composite, subdivision & Solid, liquid \\
\hline 241-AN-105 & Metals & Arsenic & $\mu \mathrm{g} / \mathrm{g}, \mu \mathrm{g} / \mathrm{L}$ & Core & Core composite, subdivision & Solid, liquid \\
\hline $241-\mathrm{AN}-105$ & Metals & Barium & $\mu \mathrm{g} / \mathrm{g}, \mu \mathrm{g} / \mathrm{L}$ & Core & Core composite, subdivision & Solid, liquid \\
\hline 241-AN-105 & Metals & Beryllium & $\mu \mathrm{g} / \mathrm{g}, \mu \mathrm{g} / \mathrm{L}$ & Core & Core composite, subdivision & Solid, liquid \\
\hline $241-\mathrm{AN}-105$ & Metals & Bismuth & $\mu \mathrm{g} / \mathrm{g}, \mu \mathrm{g} / \mathrm{L}$ & Core & Core composite, subdivision & Solid, liquid \\
\hline $241-\mathrm{AN}-105$ & Metals & Cadmium & Lgg/g, $\mu \mathrm{g} / \mathrm{L}$ & Core & Core composite, subdivision & Solid, liquid \\
\hline
\end{tabular}


Table A-2. Available and Needed Data for Required Low-Activity Waste Analyses. ${ }^{1,2}$ (41 sheets)

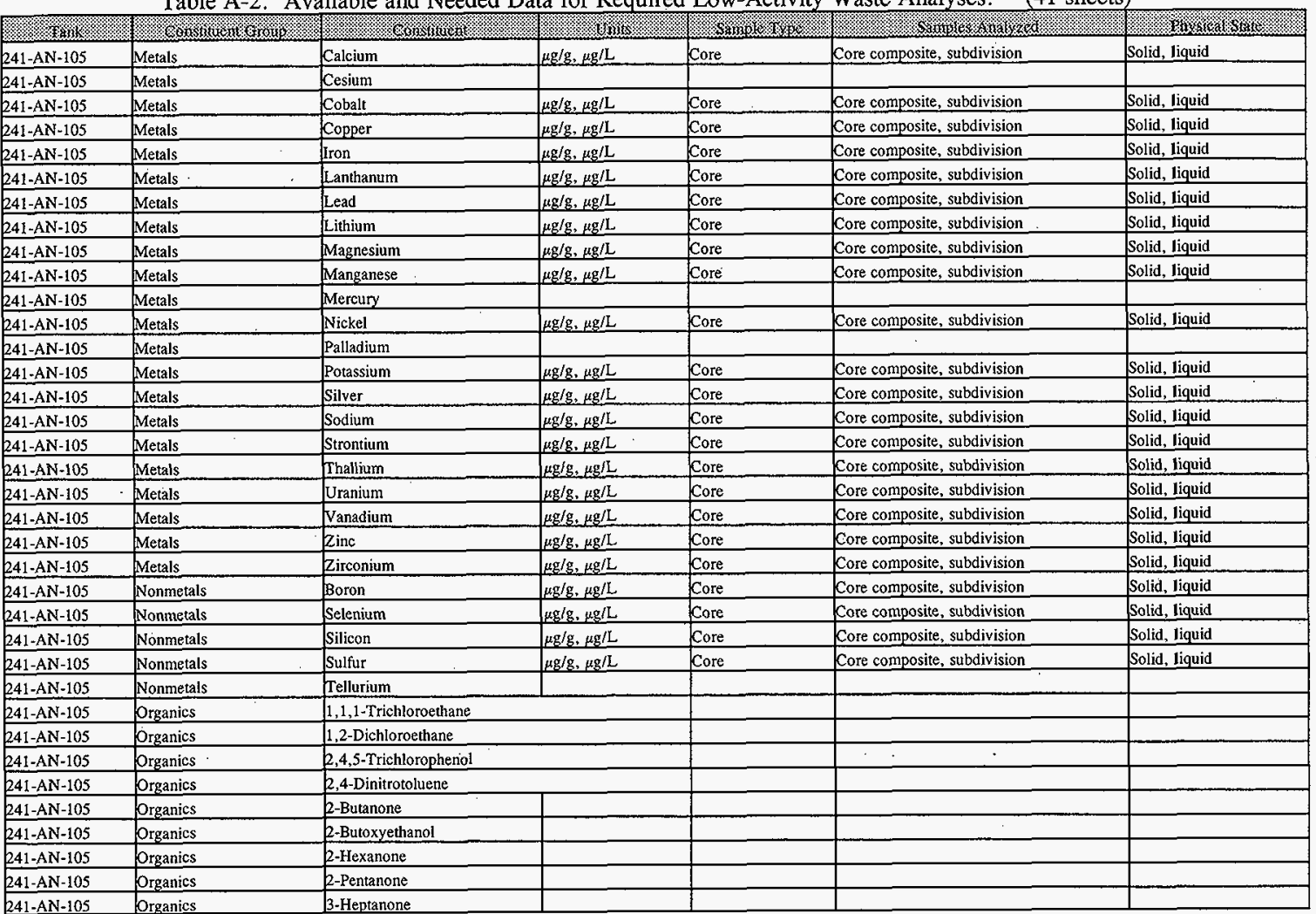


Table A-2. Available and Needed Data for Required Low-Activity Waste Analyses. ${ }^{1,2}$ (41. sheets)

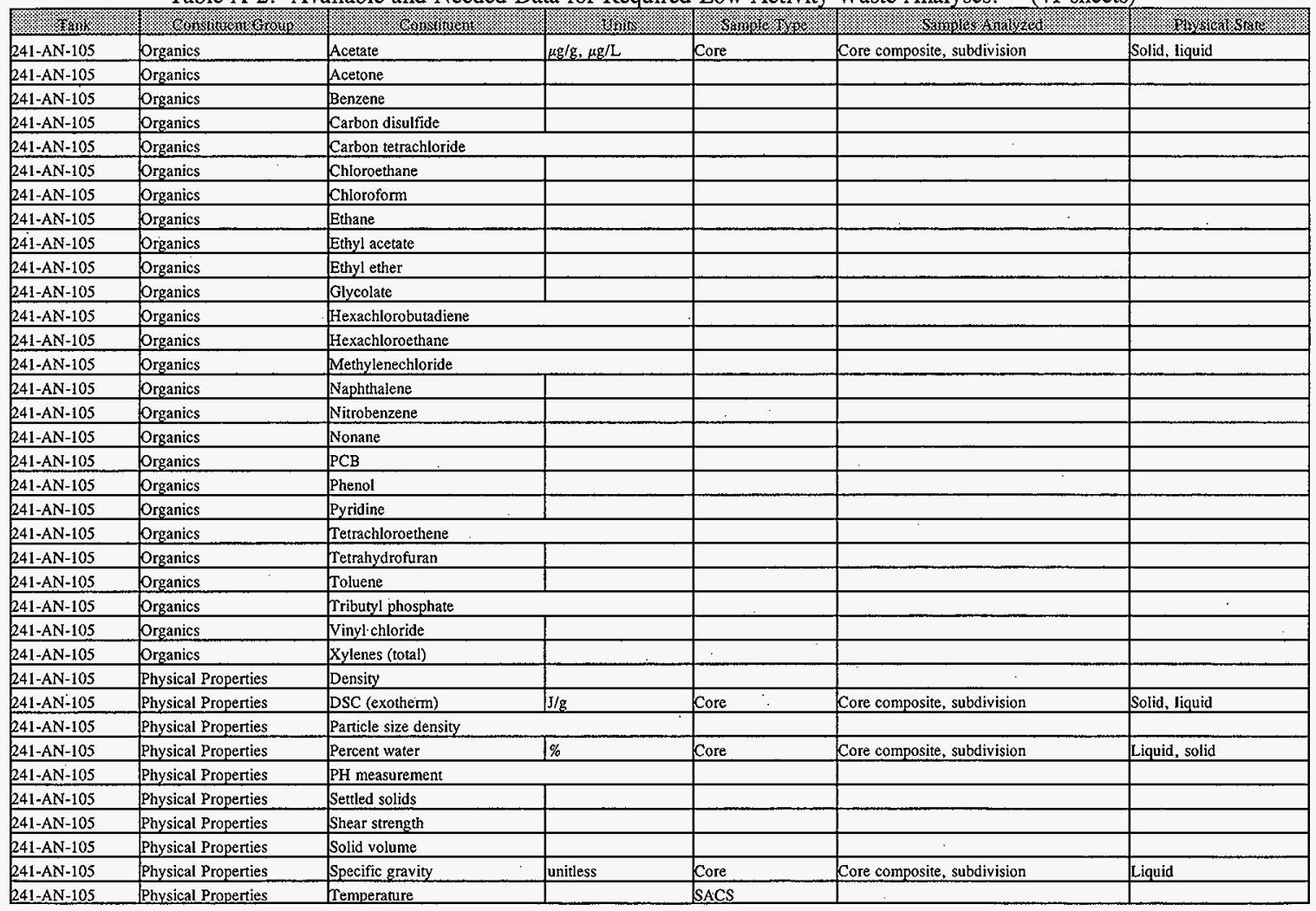


Table A-2. Available and Needed Data for Required Low-Activity Waste Analyses. ${ }^{1,2}$ (41 sheets)

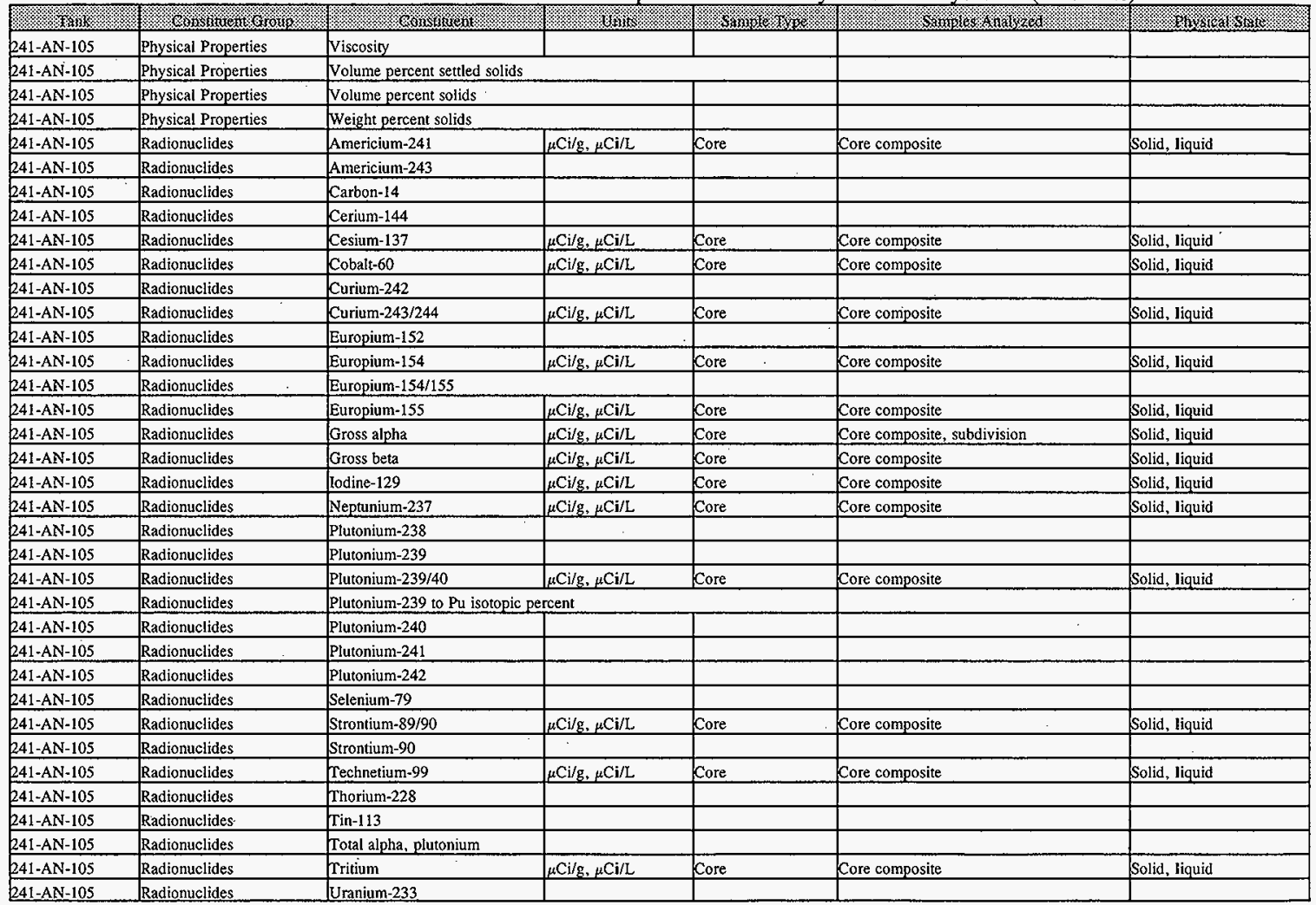


Table A-2. Available and Needed Data for Required Low-Activity Waste Analyses. ${ }^{1.2}$ (41 sheets)

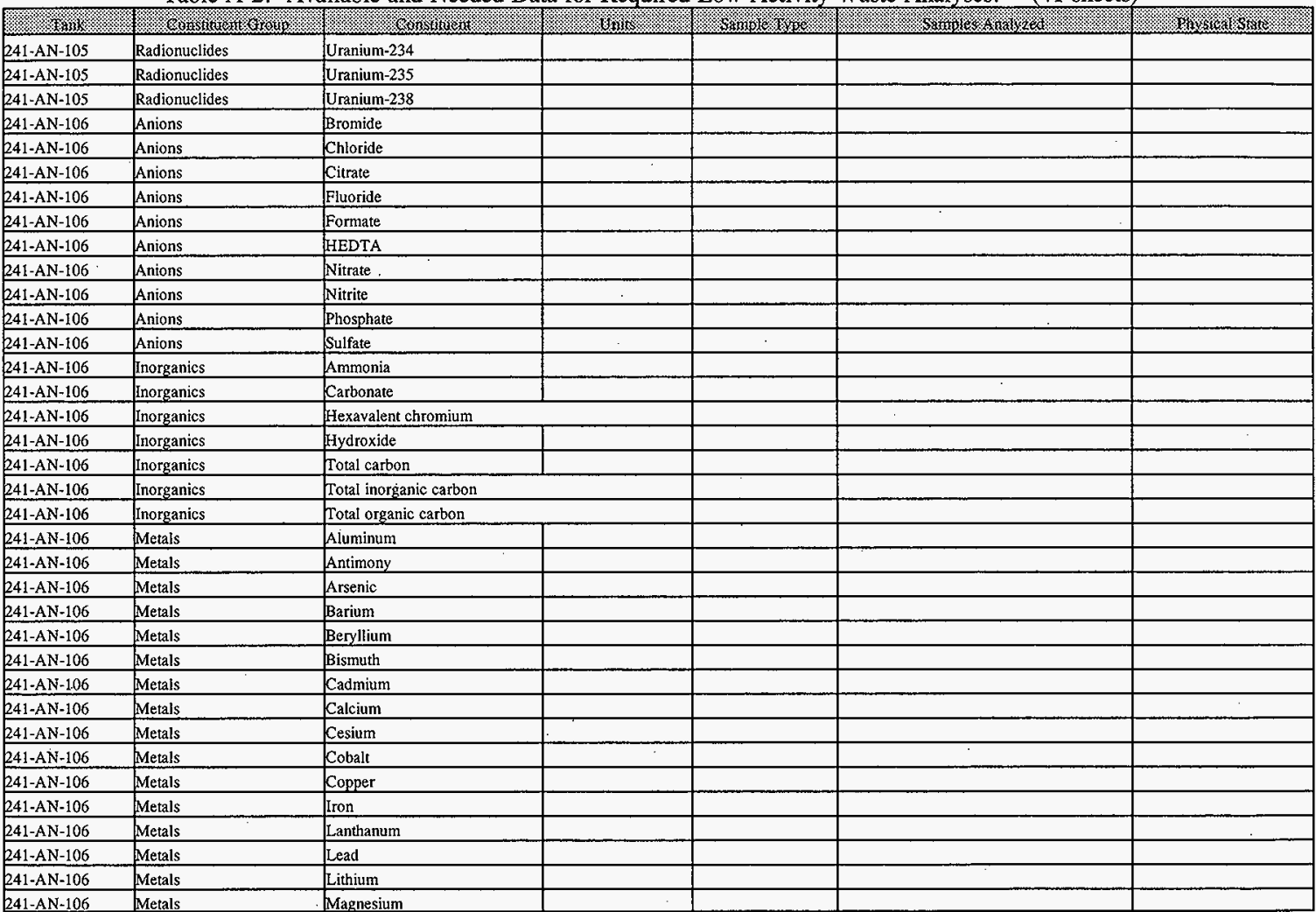


Table A-2. Available and Needed Data for Required Low-Activity Waste Analyses. ${ }^{1,2}$ (41 sheets)

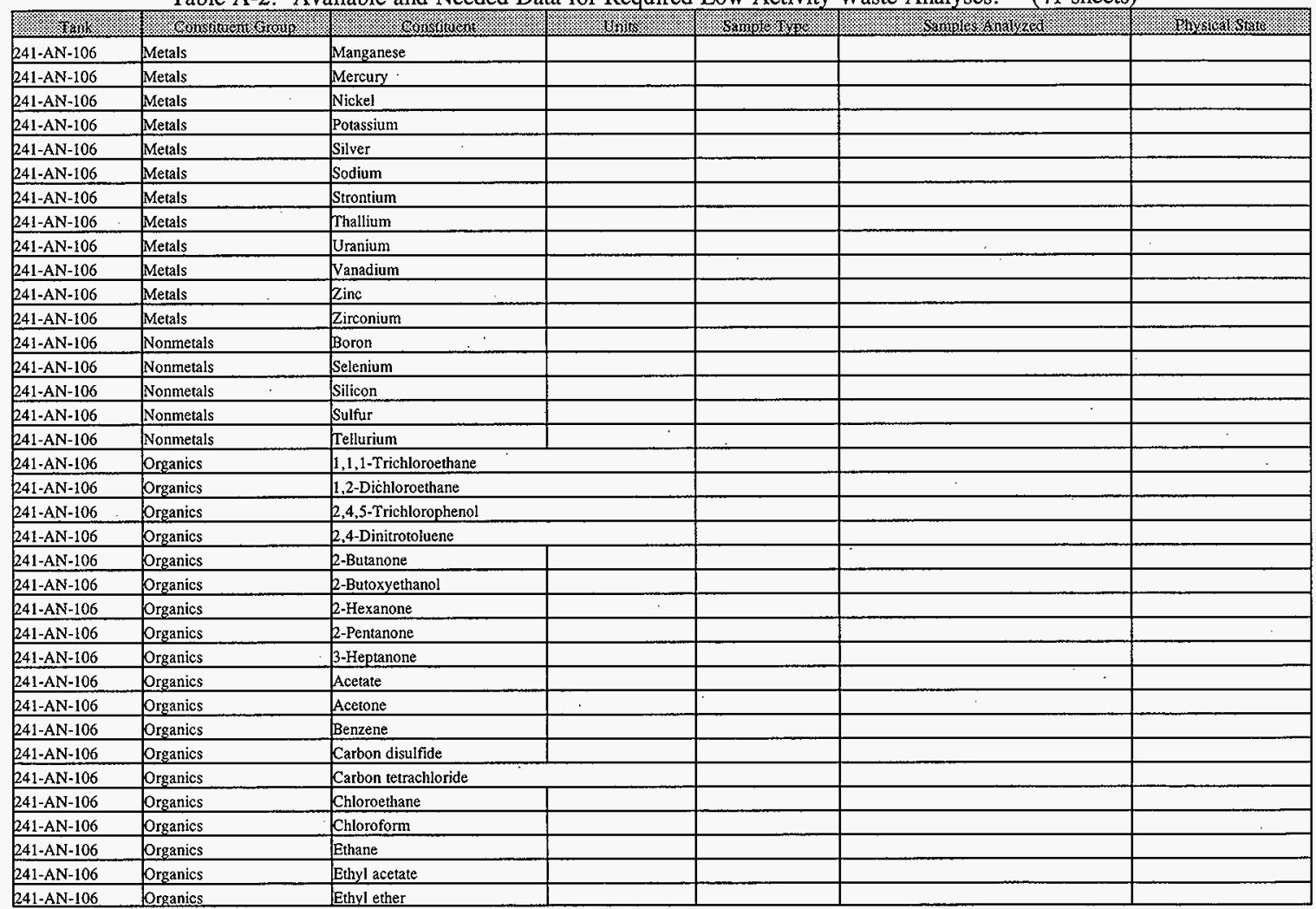


Table A-2. Available and Needed Data for Required Low-Activity Waste Analyses. ${ }^{1,2}$ (41 sheets)

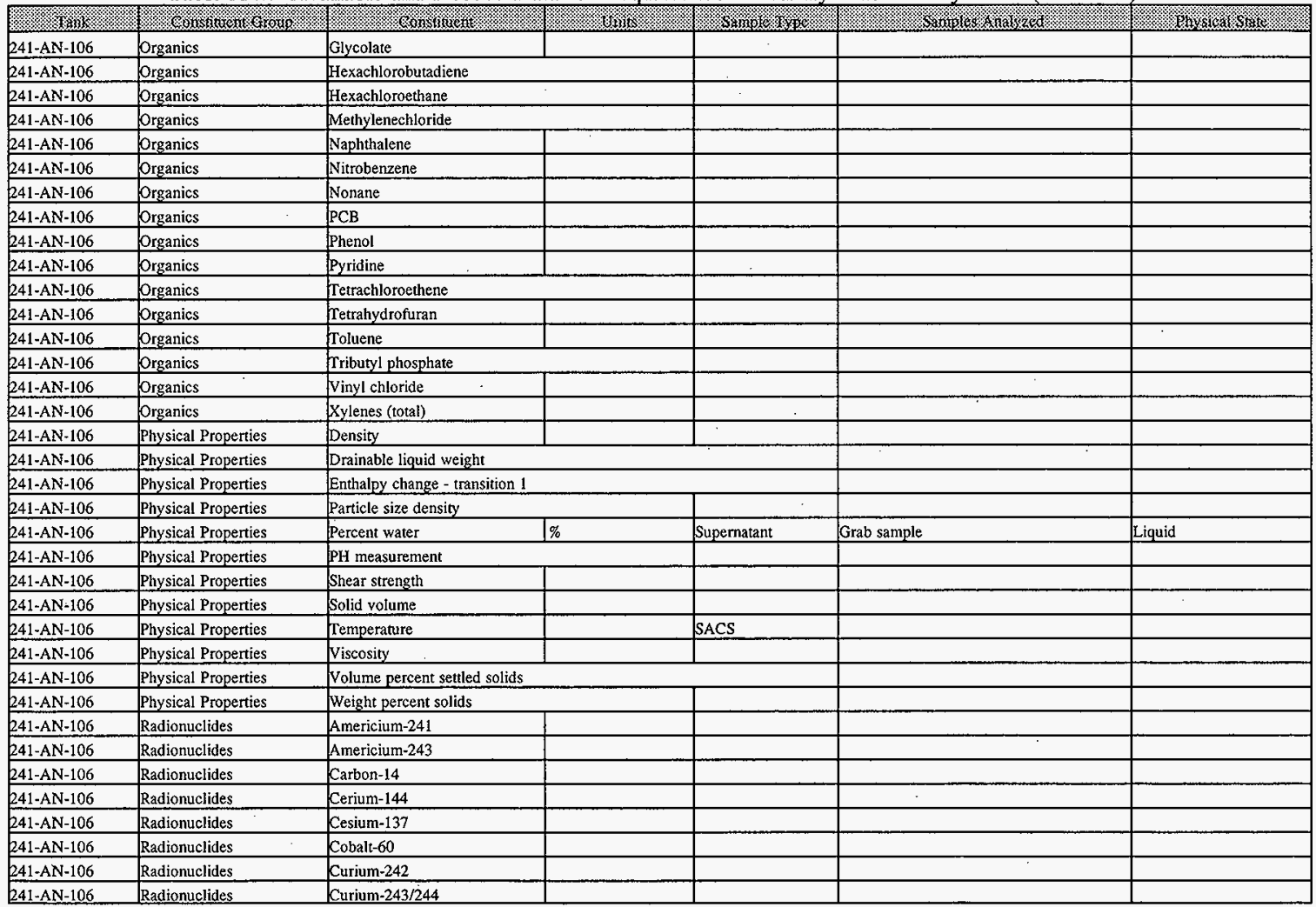


Table A-2. Available and Needed Data for Required Low-Activity Waste Analyses. ${ }^{1,2}$ (41 sheets)

\begin{tabular}{|c|c|c|c|c|c|c|}
\hline 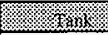 & Kox & W & Kkx/xk & 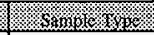 & 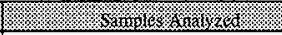 & 10 \\
\hline 241-AN-106 & Radionuclides & Europium-152 & & & & \\
\hline 241-AN-106 & Radionuclides & Europium-154 & & & & \\
\hline $241-\mathrm{AN}-106$ & Radionuclides & Europium-154/155 & & & & \\
\hline 241-AN-106 & Radionuclides & Europium-155 & & & & \\
\hline 241-AN-106 & Radionuclides & Gross alpha & $\mu \mathrm{Ci} / \mathrm{L}$ & Supernatant & Grab sample & Liquid \\
\hline 241-AN-106 & Radionuclides & Gross beta & & & & \\
\hline 241-AN-106 & Radionuclides & Iodine- 129 & & & 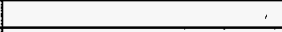 & \\
\hline 241-AN-106 & Radionuclides & Neptunium-237 & & & & \\
\hline 241-AN-106 & Radionuclides & Plutonium-238 & & & & \\
\hline 241-AN-106 & Radionuclides & Plutonium-239 & 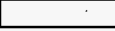 & & & \\
\hline 241-AN-106 & Radionuclides & Plutonium-239/40 & & & & \\
\hline 241-AN-106 & Radionuclides & Plutonium-240 & & & & \\
\hline 241-AN-106 & Radionuclides & Plutonium-241 & & & & \\
\hline 241-AN-106 & Radionuclides & Plutonium-242 & & & & \\
\hline 241-AN-106 & Radionuclides & Selenium-79 & & & & \\
\hline 241-AN-106 & Radionuclides & Strontium-89/90 & & & & \\
\hline 241-AN-106 & Radionuclides & Strontium-90 & & & & \\
\hline 241-AN-106 & Radionuclides & Technetium-99 & & & & \\
\hline 241-AN-106 & Radionuclides & Thorium-228 & & & & \\
\hline $241-\mathrm{AN}-106$ & Radionuclides & Tin-113 & & & & \\
\hline 241-AN-106 & Radionuclides & Tritium. & & & & \\
\hline 241-AN-106 & Radionuclides & Uranium-233 & & & . & \\
\hline 241-AN-106 & Radionuclides & Uranium-234 & & & . & \\
\hline 241-AN-106 & Radionuclides & Uranium-235 & & & & \\
\hline 241-AN-106 & Radionuclides & Uranium-238 & & . & & \\
\hline 241-AN-107 & Anions & Bromide & & & & \\
\hline 241-AN-107 & Anions & Chloride & & & & \\
\hline 241-AN-107 & Anions & Citrate & & & & \\
\hline 241-AN-107 & Anions & Fluoride & & & & \\
\hline 241-AN-107 & Anions & Formate & & & & \\
\hline 241-AN-107 & Anions & HEDTA & & & & \\
\hline 241-AN-107 & Anions & Nitrate & & & & \\
\hline 241-AN-107 & Anions & Nitrite & & & & \\
\hline 241-AN-107 & Anions & Phosphate & & & & \\
\hline 241-AN-107 & Anions & Sulfate & & & & \\
\hline 241-AN-107 & Inorganics & Ammonia & & & & \\
\hline
\end{tabular}




\begin{tabular}{|c|c|c|c|c|c|c|}
\hline & & & & uoग!Is & \$[E]əutuoN & LOI-NV-ItZ \\
\hline & & & & 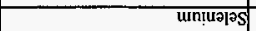 & 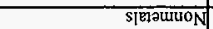 & 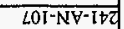 \\
\hline & & & & Horog & s[m?atuon & LOI-NV-It \\
\hline & & & & 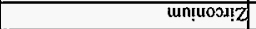 & s[घ]2W & $\angle 0 \mathrm{l}-\mathrm{NV}-\mathrm{I}+\mathrm{Z}$ \\
\hline & & & & गu!z & 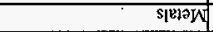 & LOI-NV-ItZ \\
\hline & & & & un!̣puz $\Lambda$ & Sमांक्से & LOI-NY-ItOC \\
\hline & & & & แn!mun & 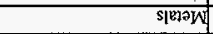 & $\angle O I-N \forall-I D Z$ \\
\hline & & & & 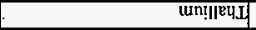 & Sकाम्य) & $\angle 01-N Y-1+0$ \\
\hline & & & & un!nuons & s[utax & $\angle 01-N \forall-I t+Z_{2}$ \\
\hline & & & & uIntpos & इमचन्स & LOI-NV-ItO \\
\hline & & & & LəAIISS & इाघण्से & LOI-NV-ItZ \\
\hline & & & & ün!ssejod & 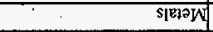 & LOI-NV-ItOC \\
\hline & & & & |әYग!N & s|man & LOI-NV-I+Z \\
\hline & & & & 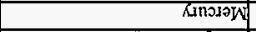 & s|हश्स & LOI-NP-ItZ \\
\hline & & & & วsautรินeW & splata & LOI-NV-ItZ \\
\hline & & & & Un!səusew & รाघวस & LOI-NV-It \\
\hline & & & & un!ุा!7] & s[m-2W & $20 \mathrm{I}-\mathrm{NV}-\mathrm{I}+\mathrm{Z}$ \\
\hline & & & & PEOT & 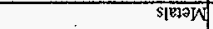 & L01-NV-1+Z \\
\hline & & & & 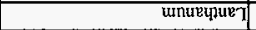 & 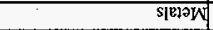 & $\angle 0 I-N \forall-I D Z$ \\
\hline & $\cdot$ & & & पर्जा & 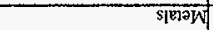 & $\angle 0 I-N \forall-I+Z$ \\
\hline & & & & daddơ & s[maw & $\angle O I-N V-I t C$ \\
\hline & & & 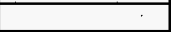 & jequa & s[1010X & $\angle 0 I-N \forall-I+Z$ \\
\hline & & & & unnisay & 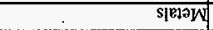 & $\angle 0 I-N V-I+Z$ \\
\hline & & & & 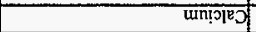 & 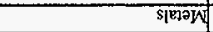 & $\angle O I-N V-I+C$ \\
\hline & & & & unliupes & S[p?JW] & LOI-NV-ItQZ \\
\hline & & & & पाnus!g| & S|lा2W & LOI-NV-ItZ \\
\hline & & & & unt!ारांग्वु & इस्य & $\left.L 0 I-N \psi^{\prime}-I \nabla Z\right]$ \\
\hline & & & . & un!se्ध & speran & LOI-NV-ItZC \\
\hline & & & & ग!ानडम & s|हागW| & $\angle 0 I-N \forall-T+2$ \\
\hline & & & & रuow!̣uज & Splaw & LOI-NV-ItZZ \\
\hline & & & & कunu!un|y & Spelow & $\angle 0 T-N \forall-T+Z$ \\
\hline p!nb!! 'PIlos & शTdurs qua & 14ืנkшadns & $7 / 3 \pi \cdot 8 / 37$ & 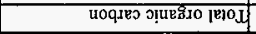 & 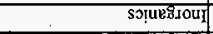 & $\angle 0 I-N V^{\prime} I t Z$ \\
\hline & & & & UOQIEO ग!uessou! |e10 I & s?̣uegrout & $\angle O T-N \forall-I t Z$ \\
\hline & & & & әрाхогркज् & 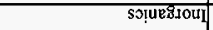 & LOI-NY-It+a \\
\hline & & & & 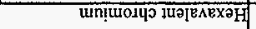 & 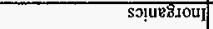 & LOI-NV-ItZ) \\
\hline & & & & ग12tioqIe & Sग!lesiouI & $\angle O I-N V-I t \theta$ \\
\hline 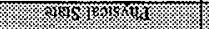 & (3) & 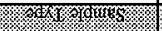 & কיক & 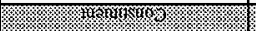 & 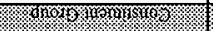 & 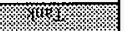 \\
\hline
\end{tabular}


Table A-2. Available and Needed Data for Required Low-Activity Waste Analyses, ${ }^{1,2}$ (41 sheets)

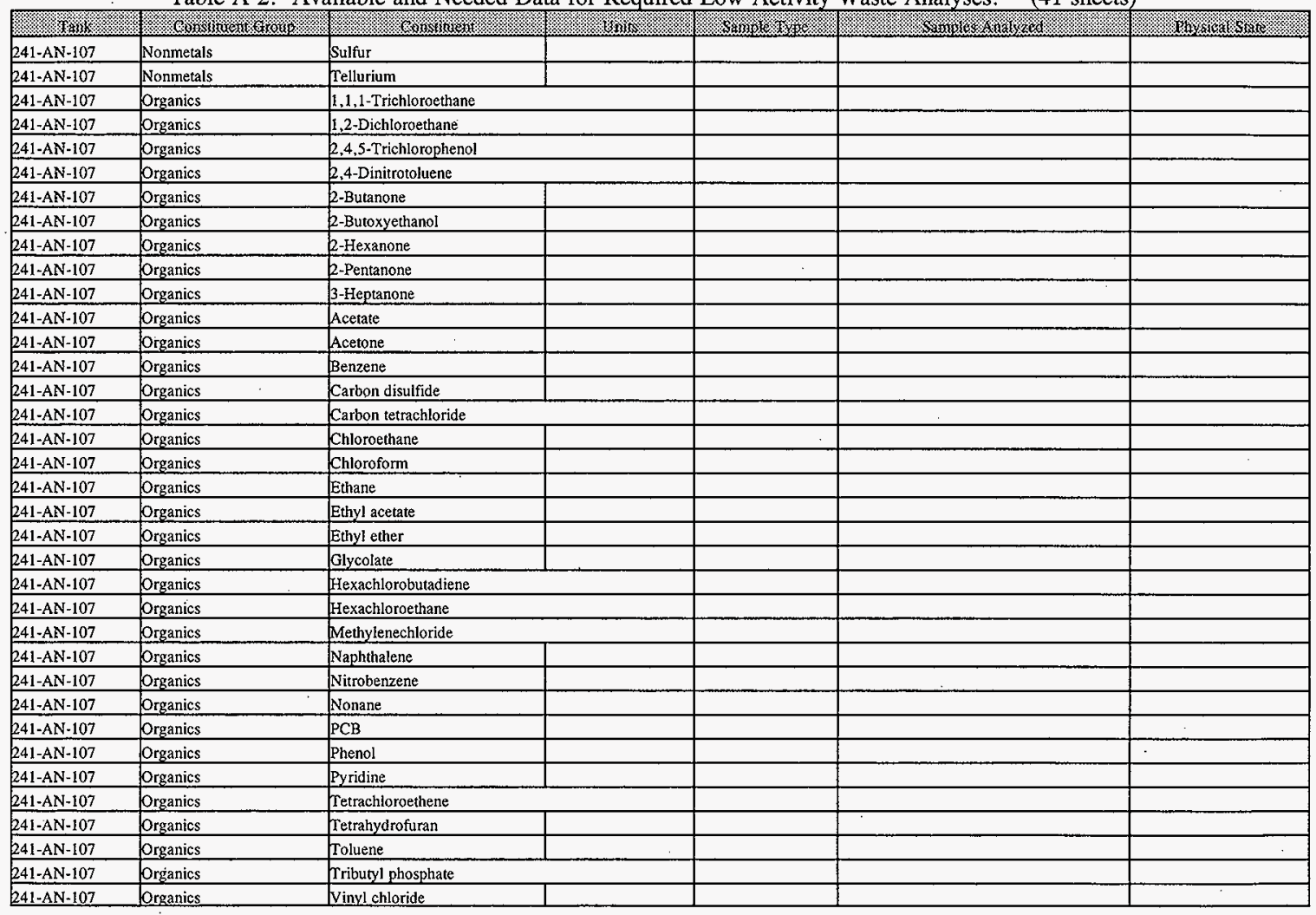


Table A-2. Available and Needed Data for Required Low-Activity Waste Analyses. ${ }^{1,2}$ (41 sheets)

\begin{tabular}{|c|c|c|c|c|c|c|}
\hline 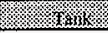 & 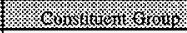 & 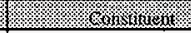 & 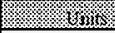 & 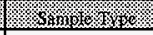 & W. & 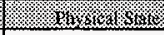 \\
\hline 241-AN-107 & Organics & Xylenes (total) & & & & \\
\hline 241-AN-107 & Physical Properties & Density & & & & \\
\hline 241-AN-107 & Physical Properties & Drainable liquid weight & & & & \\
\hline 241-AN-107 & Physical Properties & DSC (exotherm) & $\%$ & Supernatant & Grab sample & Liquid, solid \\
\hline 241-AN-107 & Physical Properties & Particle size density & & & & \\
\hline 241-AN-107 & Physical Properties & Percent water & $\%$ & Supernatant & Grab sample & Liquid, solid \\
\hline 241-AN-107 & Physical Properties & PH measurement & & & & \\
\hline 241-AN-107 & Physical Properties & Settled solids & & & & \\
\hline 241-AN-107 & Physical Properties & Shear strength & & & & \\
\hline 241-AN-107 & Physical Properties & Solid volume & & & & \\
\hline 241-AN-107 & Physical Properties & Specific gravity & unitless & Supernatant & Grab sample & Liquid \\
\hline 241-AN-107 & Physical Properties & Temperature & & SACS & & \\
\hline $241-\mathrm{AN}-107$ & Physical Properties & Viscosity & & & & Solid, liquid \\
\hline $241-\mathrm{AN}-107$ & Physical Properties & Volume percent settled & & & & \\
\hline 241-AN-107 & Physical Properties & Volume percent solids & & & & \\
\hline 241-AN-107 & Physical Properties & Weight percent solids & & & & \\
\hline 241-AN-107 & Radionuclides & Americium-241 & & & & \\
\hline 241-AN-107 & Radionuclides & Americium-243 & & & & \\
\hline 241-AN-107 & Radionuclides & Carbon-14 & & & & \\
\hline $241-A N-107$ & Radionuclides & Cerium-144 & & & & \\
\hline 241-AN-107 & Radionuclides & Cesium-137 & & & & \\
\hline 241-AN-107 & Radionuclides & Cobalt -60 & & & & \\
\hline 241-AN-107 & Radionuclides & Curium-242 & & & & \\
\hline 241-AN-107 & Radionuclides & Curium-243/244 & & & & \\
\hline 241-AN-107 & Radionuclides & Europium-152 & & & & \\
\hline 241-AN-107 & Radionuclides & Europium-154 & & & & \\
\hline 241-AN-107 & Radionuclides & Europium-154/155 & & & & \\
\hline 241-AN-107 & Radionuclides & Europium-155 & & & & \\
\hline 241-AN-107 & Radionuclides & Gross alpha & $\mu \mathrm{Ci} / \mathrm{g}, \mu \mathrm{Ci} / \mathrm{L}$ & Supernatant & Grab sample & \\
\hline 241-AN-107 & Radionuclides & Gross beta & & & & \\
\hline 241-AN-107 & Radionuclides & lodine-129 & & & & \\
\hline 241-AN-107 & Radionuclides & Neptunium-237 & & & & \\
\hline 241-AN-107 & Radionuclides & Plutonium-238 & & & & \\
\hline 241-AN-107 & Radionuclides & Plutonium-239 & & & & \\
\hline $241-\mathrm{AN}-107$ & Radionuclides & Plutonium-239/40 & & & & \\
\hline 241-AN-107 & Radionuclides & Plutonium-240 & & & & \\
\hline
\end{tabular}


Table A-2. Available and Needed Data for Required Low-Activity Waste Analyses. ${ }^{1,2}$ (41 sheets)

\begin{tabular}{|c|c|c|c|c|c|c|}
\hline (x) & 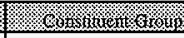 & 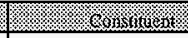 & W/x. & 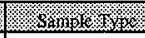 & K & 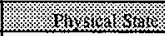 \\
\hline $241-A N-107$ & Radionuclides & Plutonium-241 & & & & \\
\hline $241 \cdot \mathrm{AN}-107$ & Radionuclides & Plutonium-242 & & & & $\therefore$ \\
\hline 241-AN-107 & Radionuclides & Selenium-79 & & & & \\
\hline 241-AN-107 & Radionuclides & Strontium-89/90 & & & & \\
\hline $241-A N-107$ & Radionuclides & Strontium-90 & & & & \\
\hline 241-AN-107 & Radionuclides & Technetium-99 & & & & \\
\hline $241-A N-107$ & Radionuclides & Thorium-228 & & & & \\
\hline 241-AN-107 & Radionuclides & $\operatorname{Tin}-113$ & & & & \\
\hline 241-AN-107 & Radionuclides & Tritium & & & & \\
\hline 241-AN-107 & Radionuclides & Uranium-233 & & & & \\
\hline 241-AN-107 & Radionuclides & Uranium-234 & & . & & \\
\hline 241-AN-107 & Radionuclides & Uranium-235 & & & & \\
\hline 241-AN-107 & Radionuclides & Uranium-238 & & & & \\
\hline $241-A W-101$ & Anions & Bromide & $\mu \mathrm{g} / \mathrm{g}, \mu \mathrm{g} / \mathrm{L}$ & Core & Core composite, subdivision & Solid, liquid \\
\hline 241-AW-101 & Anions & Chloride & $\mu \mathrm{g} / \mathrm{g}, \mu \mathrm{g} / \mathrm{L}$ & Core & Core composite & Solid, liquid \\
\hline $241-\mathrm{AW}-101$ & Anions & Citrate & & & & \\
\hline 241-AW-101 & Anions & Fluoride & $\mu \mathrm{g} / \mathrm{g}, \mu \mathrm{g} / \mathrm{L}$ & Core & Core composite & Solid, liquid \\
\hline 241-AW-101 & Anions & Formate & & & & \\
\hline 241-AW-101 & Anions & HEDTA & & & & \\
\hline 241-AW-101 & Anions & Nitrate & $\mu \mathrm{g} / \mathrm{g}, \mu \mathrm{g} / \mathrm{L}$ & Core & Core composite & Solid, liquid \\
\hline $241-\mathrm{AW}-101$ & Anions & Nitrite & $\mu \mathrm{g} / \mathrm{g}, \mu \mathrm{g} / \mathrm{L}$ & Core & Core composite & Solid, liquid \\
\hline $241-A W-101$ & Anions & Phosphate & $\mu \mathrm{g} / \mathrm{g}, \mu \mathrm{g} / \mathrm{L}$ & Core & Core composite & Solid, liquid \\
\hline 241-AW-101 & Anions & Sulfate & $\mu \mathrm{g} / \mathrm{g}, \mu \mathrm{g} / \mathrm{L}$ & Core & Core composite & Solid, liquid \\
\hline 241-AW-101 & Inorganics & Ammonia & & & & \\
\hline 241-AW-101 & Inorganics & Carbonate & & & & Solid, liquid \\
\hline 241-AW-101 & Inorganics & Hexavalent chromium & $\mu \mathrm{g} / \mathrm{g}, \mu \mathrm{g} / \mathrm{L}$ & Core & Core composite & \\
\hline 241-AW-101 & Inorganics & Hydroxide & & & & \\
\hline $241-A W-101$ & Inorganics & Total inorganic carbon & $\mu \mathrm{g} / \mathrm{g}, \mu \mathrm{g} / \mathrm{L}$ & Core & Core composite, subdivision & Solid, liquid \\
\hline 241-AW-101 & Inorganics & Total organic carbon & $\mu \mathrm{g} / \mathrm{g}, \mu \mathrm{g} / \mathrm{L}$ & Core & Core composite, subdivision & Solid, liquid \\
\hline 241-AW-101 & Metals & Aluminum & $\mu \mathrm{g} / \mathrm{g}, \mu \mathrm{g} / \mathrm{L}$ & Core & Core composite, subdivision & Solid, liquid \\
\hline 241-AW-101 & Metals & Antimony & $\mu \mathrm{g} / \mathrm{g}, \mu \mathrm{g} / \mathrm{L}$ & Core & Core composite, subdivision & Solid, liquid \\
\hline 241-AW-101 & Metals & Arsenic & $\mu \mathrm{g} / \mathrm{g}, \mu \mathrm{g} / \mathrm{L}$ & Core & Core composite, subdivision & Solid, liquid \\
\hline 241-AW-101 & Metals & Barium & $\mu \mathrm{g} / \mathrm{g}, \mu \mathrm{g} / \mathrm{L}$ & Core & Core composite, subdivision & Solid, liquid \\
\hline 241-AW-101 & Metals & Beryllium & $\mu \mathrm{g} / \mathrm{g} . \mu \mathrm{g} / \mathrm{L}$ & Core & Core composite, subdivision & Solid, liquid \\
\hline 241-AW-101 & Metals & Bismuth & $\mu \mathrm{g} / \mathrm{g}, \mu \mathrm{g} / \mathrm{L}$ & Core & Core composite, subdivision & Solid, liquid \\
\hline 241-AW-101 & Metals & Cadmium & $\mathrm{Jg} / \mathrm{g}, \mu \mathrm{g} / \mathrm{L}$ & Core & Core composite, subdivision & Solid, liguid \\
\hline
\end{tabular}


Table A-2. Available and Needed Data for Required Low-Activity Waste Analyses. ${ }^{1{ }^{12}}$ (41 sheets)

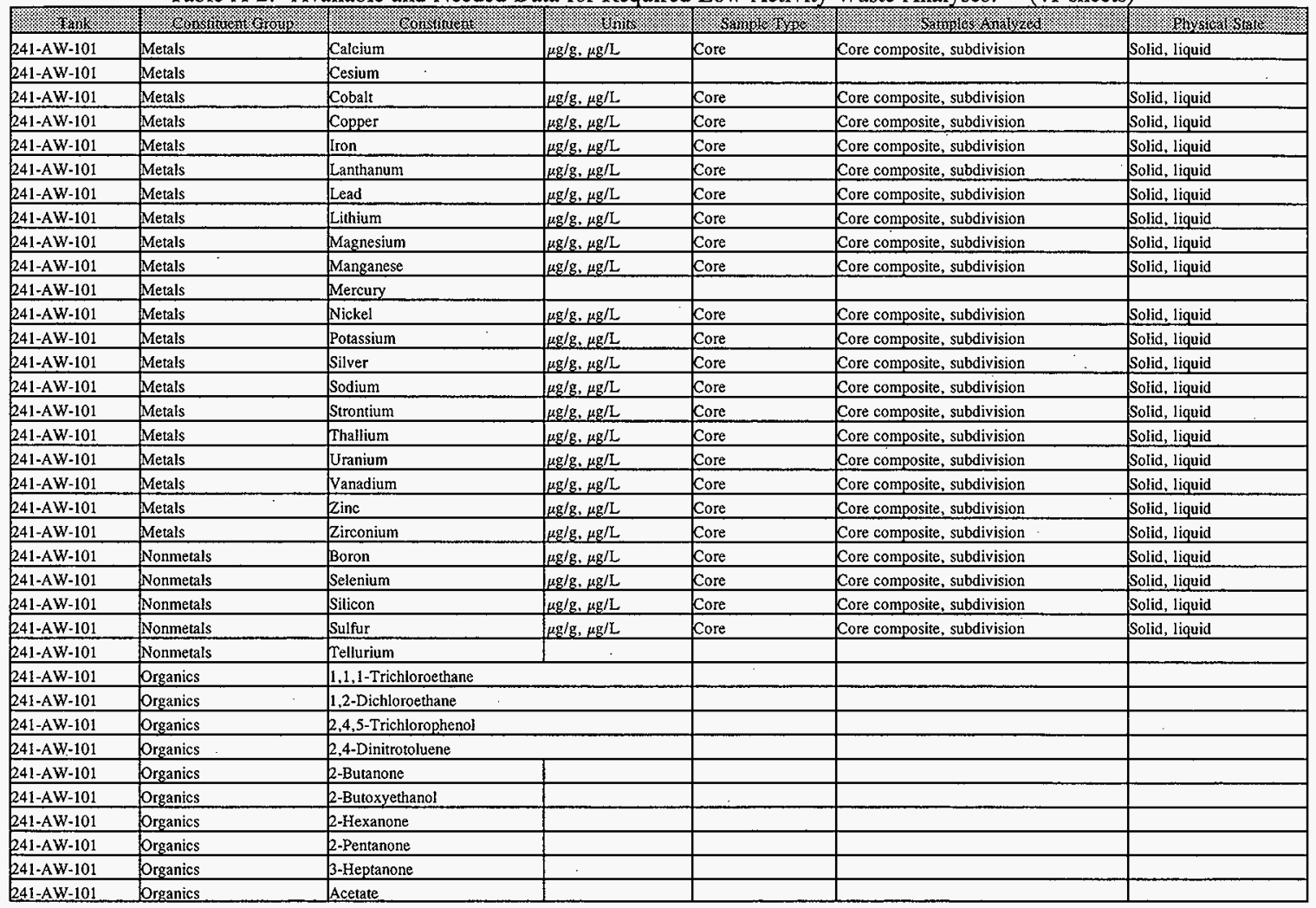


Table A-2. Available and Needed Data for Required Low-Activity Waste Analyses. ${ }^{1,2}$ (41 sheets)

\begin{tabular}{|c|c|c|c|c|c|c|}
\hline 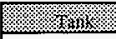 & 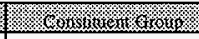 & 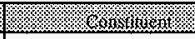 & (6) & 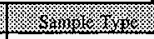 & 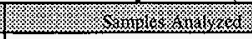 & 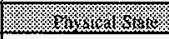 \\
\hline 241-AW-101 & Organics & Acetone & & & & \\
\hline $241-\mathrm{AW}-101$ & Organics & Benzene & & $\cdot$ & & \\
\hline 241-AW-101 & Organics & Carbon disulfide & & & & \\
\hline $241-A W-101$ & Organics & Carbon tetrachloride & & & & \\
\hline $241-A W-101$ & Organics & Chloroethane & . & & & \\
\hline 241-AW-101 & Organics & Chloroform & & & & \\
\hline $241-\mathrm{AW} \cdot 101$ & Organics & Ethane & & & & \\
\hline 241-AW-101 & Organics & Ethyl acetate & & & & \\
\hline 241-AW-101 & Organics & Ethyl ether & & & & \\
\hline 241-AW-101 & Organics & Glycolate & & & & \\
\hline $241-A W-101$ & Organics & Hexachlorobutadiene & & & & \\
\hline 241-AW-101 & Organics & Hexachloroethane & & & & \\
\hline $241-A W-101$ & Organics & Methylenechloride & & & & \\
\hline $241-\mathrm{AW}-101$ & Organics & Naphthalene & & & & \\
\hline 241-AW-101 & Organics & Nitrobenzene & & & & \\
\hline 241-AW-101 & Organics & Nonane & & & & \\
\hline $241-\mathrm{AW}-101$ & Organics & $\mathrm{PCB}$ & & . & & \\
\hline 241-AW-101 & Organics & Phenol & & & & $\overline{5}$ \\
\hline 241-AW-101 & Organics & Pyridine & & & & \\
\hline 241-AW-101 & Organics & Tetrachloroethene & & & & \\
\hline 241-AW-101 & Organics & Tetrahydrofuran & & & & \\
\hline 241-AW-101 & Organics & Toluene & & & & \\
\hline 241-AW-101 & Organics & Tributyl phosphate & & & & \\
\hline 241-AW-101 & Organics & Vinyl chloride & & & & \\
\hline 241-AW-101 & Organics & Xylenes (total) & & & & \\
\hline $241-\mathrm{AW}-101$ & Physical Properties & Density & & & & \\
\hline 241-AW-101. & Physical Properties & Drainable liquid weight & & & & \\
\hline 241-AW-101 & Physical Properties & DSC (exotherm) & $\%$ & Core & Core composite, subdivision & Liquid, solid \\
\hline 241-AW-101 & Physical Properties & Particle size density & & & & \\
\hline 241-AW-10I & Physical Properties & Pércent water & $\%$ & Core & Core composite, subdivision & Liquid, solid \\
\hline $241-\mathrm{AW}-101$ & Physical Properties & $\mathrm{PH}$ measurement & & & & \\
\hline $241-\mathrm{AW}-101$ & Physical Properties & Settled solids & & & & \\
\hline $241-\mathrm{AW}-101$ & Physical Properties & Shear strength & & & & \\
\hline 241-AW-101 & Physical Properties & Solid volume & & & & \\
\hline $241-\mathrm{AW}-101$ & Physical Properties & Specific gravity & unitless & Core & Core composite, subdivision & Liquid \\
\hline $241-A W-101$ & Physical Properties & Temperature & & SACS & & \\
\hline
\end{tabular}


Table A-2. Available and Needed Data for Required Low-Activity Waste Analyses. ${ }^{1,2}$ (41 sheets)

\begin{tabular}{|c|c|c|c|c|c|c|}
\hline 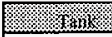 & 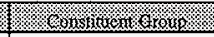 & 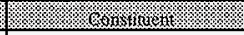 & 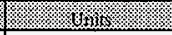 & 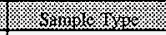 & (3) & 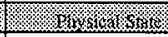 \\
\hline 241-AW-101 & Physical Properties & Viscosity & & & & \\
\hline 241-AW-101 & Physical Properties & Volume percent settled solids & & & & \\
\hline 241-AW-101 & Physical Properties & Volume percent solids & & . & & \\
\hline 241-AW-101 & Physical Properties & Weight percent solids & & & & \\
\hline $241-\mathrm{AW}-101$ & Radionuclides & Americium-241 & $\mu \mathrm{Ci} / \mathrm{g}, \mu \mathrm{Ci} / \mathrm{L}$ & Core & Core composite & Solid, liquid \\
\hline 241-AW-101 & Radionuclides & Americium-243 & & & & \\
\hline 241-AW-101 & Radionuclides & Carbon-14 & & & & \\
\hline $241-\mathrm{AW}-101$ & Radionuclides & Cerium-144 & & & & \\
\hline $241-A W-101$ & Radionuclides & Cesium-137 & $\mu \mathrm{Ci} / \mathrm{g}, \mu \mathrm{Ci} / \mathrm{L}$ & Core & Core composite & Solid, liquid \\
\hline 241-AW-101 & Radionuclides & Cobalt -60 & $\mu \mathrm{Ci} / g, \mu \mathrm{Ci} / \mathrm{L}$ & Core & Core composite & Solid, liquid \\
\hline 241-AW-101 & Radionuclides & Curium-242 & & & & \\
\hline $241-\mathrm{AW}-101$ & Radionuclides & Curium-243/244 & $\mu \mathrm{Ci} / \mathrm{g}, \mu \mathrm{Ci} / \mathrm{L}$ & Core & Core composite & Solid, liquid \\
\hline 241-AW-101 & Radionuclides & Europium-152 & & & & \\
\hline $241-\mathrm{AW}-101$ & Radionuclides & Europium-154 & $\mu \mathrm{Ci} / \mathrm{g}, \mu \mathrm{Ci} / \mathrm{L}$ & Core & Core composite & Solid, liquid \\
\hline 241-AW-101 & Radionuclides & Europium-154/155 & & & & \\
\hline $241-A W-101$ & Radionuclides & Europium-155 & ${ }_{\mu} \mathrm{Ci} / \mathrm{g}, \mu \mathrm{Ci} / \mathrm{L}$ & Core & Core composite & Solid, liquid \\
\hline $241-A \mathrm{~W}-101$ & Radionuclides & Ǵross alpha & $\mu \mathrm{Ci} / \mathrm{g}, \mu \mathrm{Ci} / \mathrm{L}$ & Core & Core composite, subdivision & Solid, liquid \\
\hline $241-\mathrm{AW}-101$ & Radionuclides & Gross beta & $\mu \mathrm{Ci} / \mathrm{g}, \mu \mathrm{Ci} / \mathrm{L}$ & Core & Core composite & Solid, liquid \\
\hline $241-\mathrm{AW}-101$ & Radionuclides & lodine-129 & $\mu \mathrm{Ci} / \mathrm{g}$ & Core & Core composite & Solid \\
\hline $241-\mathrm{AW}-101$ & Radionuclides & Neptunium-237 & & & & \\
\hline 241-AW-101 & Radionuclides & Plutonium-238 & & & & \\
\hline $241-A \mathrm{~W}-101$ & Radionuclides & Plutonium-239 & & & & . \\
\hline $241-A W-101$ & Radionuclides & Plutonium-239/40 & $\mu \mathrm{Ci} / \mathrm{g}, \mu \mathrm{Ci} / \mathrm{L}$ & Core & Core composite & Solid, liquid \\
\hline $241-A W-101$ & Radionuclides & Plutonium-240 & & & & \\
\hline 241-AW-101 & Radionuclides & Plutonium-241 & & & & \\
\hline 241-AW-101 & Radionuclides & Plutonium-242 & & & & \\
\hline $241-\mathrm{AW}-101$ & Radionuclides & Selenium-79 & & & & \\
\hline $241-\mathrm{AW}-101$ & Radionuclides & Strontium-89/90 & $\mu \mathrm{Ci} / \mathrm{g}, \mu \mathrm{Ci} / \mathrm{L}$ & Core & Core composite & Solid, liquid \\
\hline $241-\mathrm{AW}-101$ & Radionuclides & Strontium-90 & & & & \\
\hline 241-AW-101 & Radionuclides & Technetium-99 & ${ }_{\mu} \mathrm{Ci} / \mathrm{g}, \mu \mathrm{Ci} / \mathrm{L}$ & Core & Core composite & Solid, liquid \\
\hline $241-A W-101$ & Radionuclides & Thorium-228 & & & & \\
\hline $241-A W-101$ & Radionuclides & Tin-113 & & & & \\
\hline 241-AW-101 & Radionuclides & Tritium & $\mu \mathrm{Ci} / \mathrm{L}$ & Core & Core composite & Liquid \\
\hline $241-\mathrm{AW}-101$ & Radionuclides & Uranium-233 & . & & & \\
\hline $241-\mathrm{AW}-10 \mathrm{I}$ & Radionuclides & Uranium-234 & & & & \\
\hline $241-\mathrm{AW}-101$ & Radionuclides & Uranium-235 & & & & \\
\hline
\end{tabular}


Table A-2. Available and Needed Data for Required Low-Activity Waste Analyses. ${ }^{1,2}$ (41 sheets)

\begin{tabular}{|c|c|c|c|c|c|c|}
\hline 1.x. & \% & \% & sinim & s. & 3inindix & 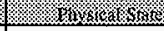 \\
\hline $241-\mathrm{AW}-101$ & Radionuclides & Uranium-238 & & & & \\
\hline 241-AZ-101 & Anions & Bromide & & & & \\
\hline $241-A Z-101$ & Anions & Chloride & $\mu \mathrm{g} / \mathrm{L}$ & Supernatant & Grab sample & Liquid \\
\hline 241-AZ-101 & Anions & Citrate & & & & \\
\hline $241-\mathrm{AZ}-101$ & Anions & Fluoride & $\mu \mathrm{g} / \mathrm{L}$ & Supernatant & Grab sample & Liquid \\
\hline 241-AZ-101 & Anions & Formate & & & & \\
\hline $241-\mathrm{AZ}-101$ & Anions & HEDTA & & & & \\
\hline 241-AZ-101 & Anions & Nitrate & $\mu \mathrm{g} / \mathrm{L}$ & Supernatant & Grab sample & Liquid \\
\hline $241-A Z-101$ & Anions: & Nitrite & $\mu \mathrm{g} / \mathrm{L}$ & Supernatant & Grab sample & Liquid \\
\hline $241-\mathrm{AZ}-101$ & Anions & Phosphate & $\mu \mathrm{g} / \mathrm{L}$ & Supernatant & Grab sample & Liguid \\
\hline 241-AZ-101 & Anions & Sulfate & $\mu \mathrm{g} / \mathrm{L}$ & Supernatant & Grab sample & Liquid \\
\hline $241-A Z-101$ & Inorganics & Ammonia & & & & \\
\hline $241-\mathrm{AZ}-101$ & Inorganics & Carbonate & & & & \\
\hline $241-\mathrm{AZ}-101$ & Inorganics & Hexavalent chromium & & & & \\
\hline 241-AZ-101 & Inorganics & Hydroxide & $\mu \mathrm{g} / \mathrm{L}$ & Supernatant & Grab sample & Liquid \\
\hline $241-A Z-101$ & Inorganics & Total inorganic carbon & $\mu \mathrm{g} / \mathrm{L}$ & Supernatant & Grab sample & Liquid \\
\hline 241-AZ-101 & Inorganics & Total organic carbon & $\mu \mathrm{g} / \mathrm{L}$ & Superuatant & Grab sample & Liquid \\
\hline $241-A Z-101$ & Metals & Aluminum & $\mu \mathrm{g} / \mathrm{L}$ & Supernatant & Grab sample & Liquid \\
\hline $241-A Z-101$ & Metals & Antimony & & & & \\
\hline 241-AZ-101 & Metals & Arsenic & & & & \\
\hline $241-A Z-101$ & Metals & Barium & & & & \\
\hline 241-AZ-101 & Metals & Beryllium & & & & \\
\hline $241-\mathrm{AZ}-101$ & Metals & Bismuth & & & & \\
\hline 241-AZ-101 & Metals & Cadmium & & & & \\
\hline $241-\mathrm{AZ}-101$ & Metals & Calcium & & & & \\
\hline 241-AZ-101 & Metals & Cesium & & & & \\
\hline $241-\mathrm{AZ}-101$ & Metals & Cobalt & $:$ & & & \\
\hline 241-AZ-101 & Metals & Copper & & & & \\
\hline $241-\mathrm{AZ}-101$ & Metals & Iron & $\mu \mathrm{g} / \mathrm{L}$ & Supernatant & Grab sample & Liquid \\
\hline $241-A Z-101$ & Metals & Lanthanum & & & & \\
\hline $241-\mathrm{AZ}-101$ & Metals & Lead & & & & \\
\hline 241-AZ-101 & Metals & Lithium & & & & \\
\hline $241-\mathrm{AZ}-101$ & Metals & Magnesium & & & & \\
\hline $241-A Z-101$ & Metals & Manganese & & & & \\
\hline 241-AZ-101 & Metals & Mercury & & & & \\
\hline 241-AZ-101 & Metals & Nickel & & & & \\
\hline
\end{tabular}


Table A-2. Available and Needed Data for Required Low-Activity Waste Analyses. ${ }^{1,2}$ (41 sheets)

\begin{tabular}{|c|c|c|c|c|c|c|}
\hline 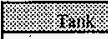 & 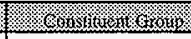 & 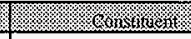 & W. & 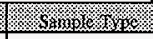 & W. & 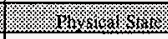 \\
\hline $241-\mathrm{AZ}-101$ & Metals & Potassium & & & & \\
\hline $241-\mathrm{AZ}-101$ & Metals & Silver & & & 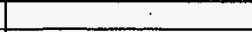 & \\
\hline 241-AZ-101 & Metals & Sodium & $\mu \mathrm{g} / \mathrm{L}$ & Supernatant & Grab sample & Liquid \\
\hline $241-\mathrm{AZ}-10 \mathrm{I}$ & Metals & Strontium & & & & \\
\hline $241-A Z-101$ & Metals & Thallium & & & & \\
\hline $241-A Z-10 I$ & Metals & Uranium & & & & \\
\hline $241-\mathrm{AZ}-101$ & Metals & Vanadium & & & & \\
\hline 241-AZ-101 & Metals & Zinc & & & & \\
\hline $241-A Z-10 \mathrm{I}$ & Metals & Zirconium & & & & \\
\hline 241-AZ-10I & Nonmetals & Boron & & & & \\
\hline 241-AZ-101 & Nonmetals & Selenium & & & & \\
\hline $241-\mathrm{AZ}-101$ & Nonmetals & Silicon & & & & \\
\hline $241-\mathrm{AZ}-101$ & Nonmetals & Sulfur & & & & \\
\hline $241-A Z-101$ & Nonmetals & Tellurium & & & & \\
\hline $241-A Z-101$ & Organics & 1,1,1-Trichloroethane & & & & \\
\hline $241-\mathrm{AZ}-101$ & Organics & 1,2-Dichloroethane & & & & \\
\hline $241-A Z-101$ & Organics & 2,4,5-Trichlorophenol & & & & \\
\hline $241-A Z-101$ & Organics & 2,4-Dinitrotoluene & & & & \\
\hline 241-AZ-10I & Organics & 2-Butanone & & & & \\
\hline $241-\mathrm{AZ}-101$ & Organics & 2-Butoxyethanol & & & & \\
\hline $241-A Z-101$ & Organics & 2-Hexanone & & & & \\
\hline $241-A Z-101$ & Organics & 2-Pentanone & & & & \\
\hline $241-A Z-101$ & Organics & 3-Heptanone & & & & \\
\hline $241-A Z-101$ & Organics & Acetate & & & & \\
\hline 241-AZ-101 & Organics & Acetone & & & & \\
\hline $241-\mathrm{AZ}-101$ & Organics & Benzene & & & & \\
\hline $241-A Z-101$ & Organics & Carbon disulfide & & & & \\
\hline $241-A Z-101$ & Organics & Carbon tetrachloride & & & & \\
\hline 241-AZ-101 & Organics & Chloroethane & & & & \\
\hline $241-A Z-101$ & Organics & Chloroform & & & & \\
\hline $241-\mathrm{AZ}-101$ & Organics & Ethane & & & & \\
\hline 241-AZ-101 & Organics & Ethyl acetate & & & & \\
\hline $241-A Z-101$ & Organics & Ethyl ether & & & & \\
\hline 241-AZ-101 & Örganics & Glycolate & & & & \\
\hline $24 !-A Z-101$ & Organics & Hexachlorobutadiene & & & & \\
\hline 241-AZ-101 & Organics & Hexachloroethane & & & & \\
\hline
\end{tabular}


Table A-2. Available and Needed Data for Required Low-Activity Waste Analyses. ${ }^{1,2}$ (41 sheets)

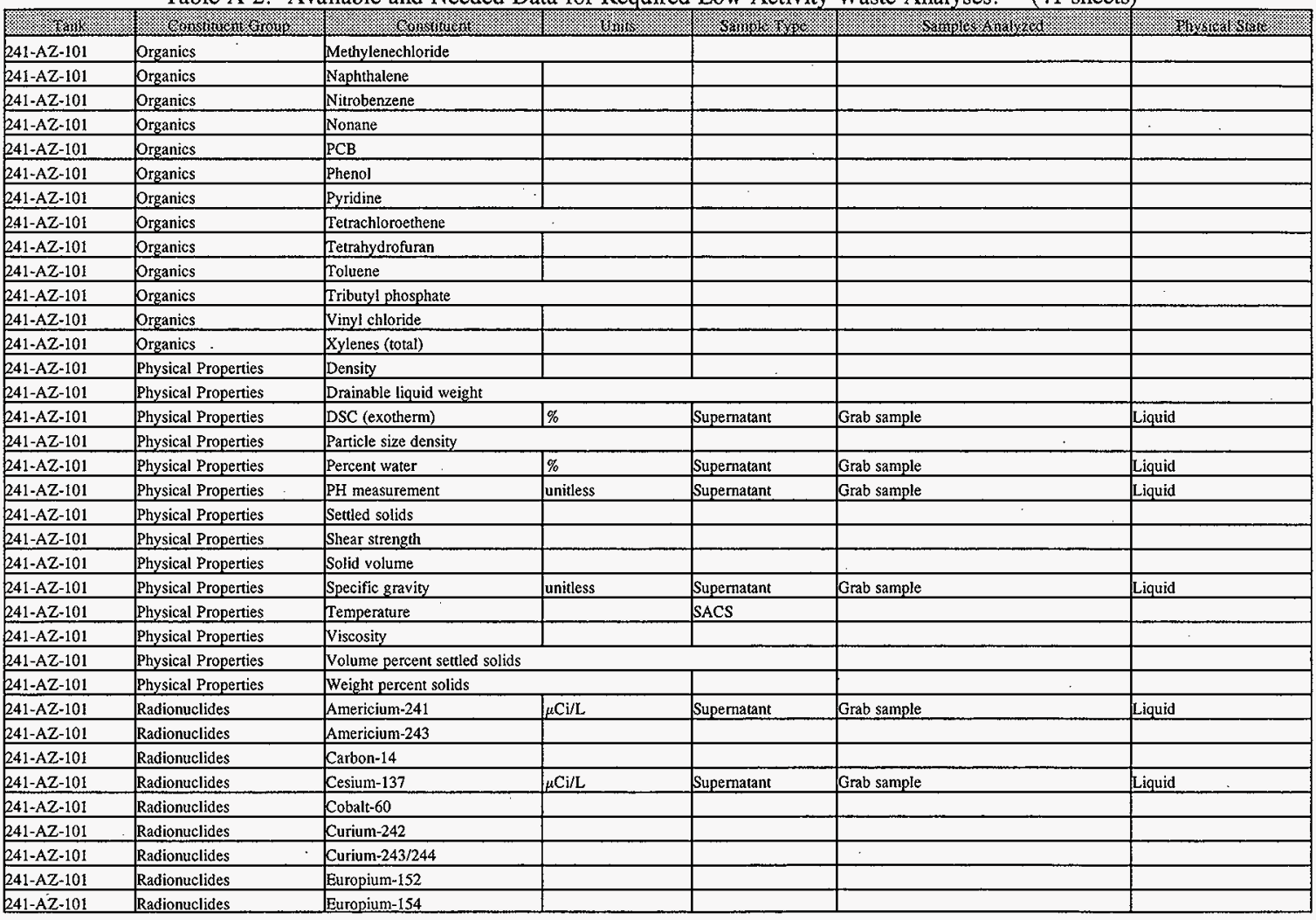


Table A-2. Available and Needed Data for Required Low-Activity Waste Analyses, ${ }^{1,2}$ (41 sheets)

\begin{tabular}{|c|c|c|c|c|c|c|}
\hline 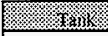 & 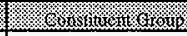 & W & 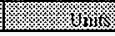 & 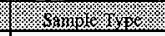 & 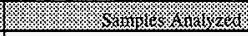 & 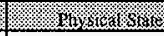 \\
\hline $241-A Z-101$ & Radionuclides & Europium-154/155 & & & & \\
\hline $241-A Z-101$ & Radionuclides & Europium-155 & & & & \\
\hline $241-\mathrm{AZ}-101$ & Radionuclides & Gross alpha & & & & \\
\hline 241-AZ-101 & Radionuclides & Gross beta &. & & & \\
\hline $241-A Z-101$ & Radionuclides & Iodine-129 & & & & \\
\hline 241-AZ-101 & Radionuclides & Neptunium-237 & & & & \\
\hline $241-A Z-101$ & Radionuclides & Plutonium-238 & $\mu \mathrm{Ci} / \mathrm{L}$ & Supernatant & Grab sample & Liquid \\
\hline 241-AZ-101 & Radionuclides & Plutonium-239 & & & & \\
\hline $241-A Z-101$ & Radionuclicies & Plutonium-239/40 & $\mu \mathrm{Ci} / \mathrm{L}$ & Supematant & Grab sample & Liquid \\
\hline 241-AZ-101 & Radionuclides & Plutonim-238 & & & & \\
\hline $24 \mathrm{I}-\mathrm{AZZ}-101$ & Radionuclides & Plutonium-240 & & & & \\
\hline $241-\mathrm{AZ}-101$ & Radionuclides & Plutonium-241 & & & & \\
\hline 241-AZ-101 & Radionuclides & Plutonium-242 & & & & \\
\hline $241-\mathrm{AZ}-101$ & Radionuclides & Selenium-79 & & & & \\
\hline 241-AZ-101 & Radionuclides & Strontium-89/90 & & & & \\
\hline 241-AZ-101 & Radionuclides & Strontium-90 & $\mu \mathrm{Ci} / \mathrm{L}$ & Supernatant & Grab sample & Liquid \\
\hline 241-AZ-101 & Radionuclides & Technetium-99 & & & & \\
\hline 241-AZ-101 & Radionuclides & Thorium-228 & & & & \\
\hline 241-AZ-101 & Radionuclides & Tin-113 & & & & \\
\hline $241-A Z-101$ & Radionuclides & Tritium & & & & \\
\hline 241-AZ-101 & Radionuclides & Uranium-233 & & & & \\
\hline 241-AZ-101 & Radionuclides & Uranium-234 & & & & \\
\hline 241-AZ-101 & Radionuclides & Uranium-235 & & & & \\
\hline 241-AZ-101 & Radionuclides & Uranium-238 & & & & \\
\hline $241-\mathrm{AZ}-102$ & Anions & Bromide & & & & \\
\hline $241-A Z-102$ & Anions & Chloride & $\mu \mathrm{g} / \mathrm{L}$ & Supernatant & Grab sample & Liquid \\
\hline $241-\mathrm{AZ}-102$ & Anions & Citrate & & & & \\
\hline 241-AZ-102 & Anions & Fluoride & $\mu \mathrm{g} / \mathrm{L}$ & Supernatant & Grab sample & Liquid \\
\hline $241-A Z-102$ & Anions & Formate & & & & \\
\hline $241-\mathrm{AZ}-102$ & Anions & HEDTA & & & & \\
\hline $241-\mathrm{AZ}-102$ & Anions & Nitrate & $\mu \mathrm{g} / \mathrm{L}$ & Supernatant & Grab sample & Liquid \\
\hline $241-A Z-102$ & Anions & Nitrite & $\mu \mathrm{g} / \mathrm{L}$ & Supernatant & Grab sample & Liquid \\
\hline $241-\mathrm{AZ}-102$ & Anions & Phosphate & $\mu \mathrm{g} / \mathrm{L}$ & Supernatant & Grab sample & Liquid \\
\hline $241-A Z-102$ & Anions & Sulfate & $\mu \mathrm{g} / \mathrm{L}$ & Supernatant & Grab sample & Liquid \\
\hline $241-A Z-102$ & Inorganics & Ammonia & & & & \\
\hline 241-AZ-102 & Inorganics & Carbonate & & & & \\
\hline
\end{tabular}




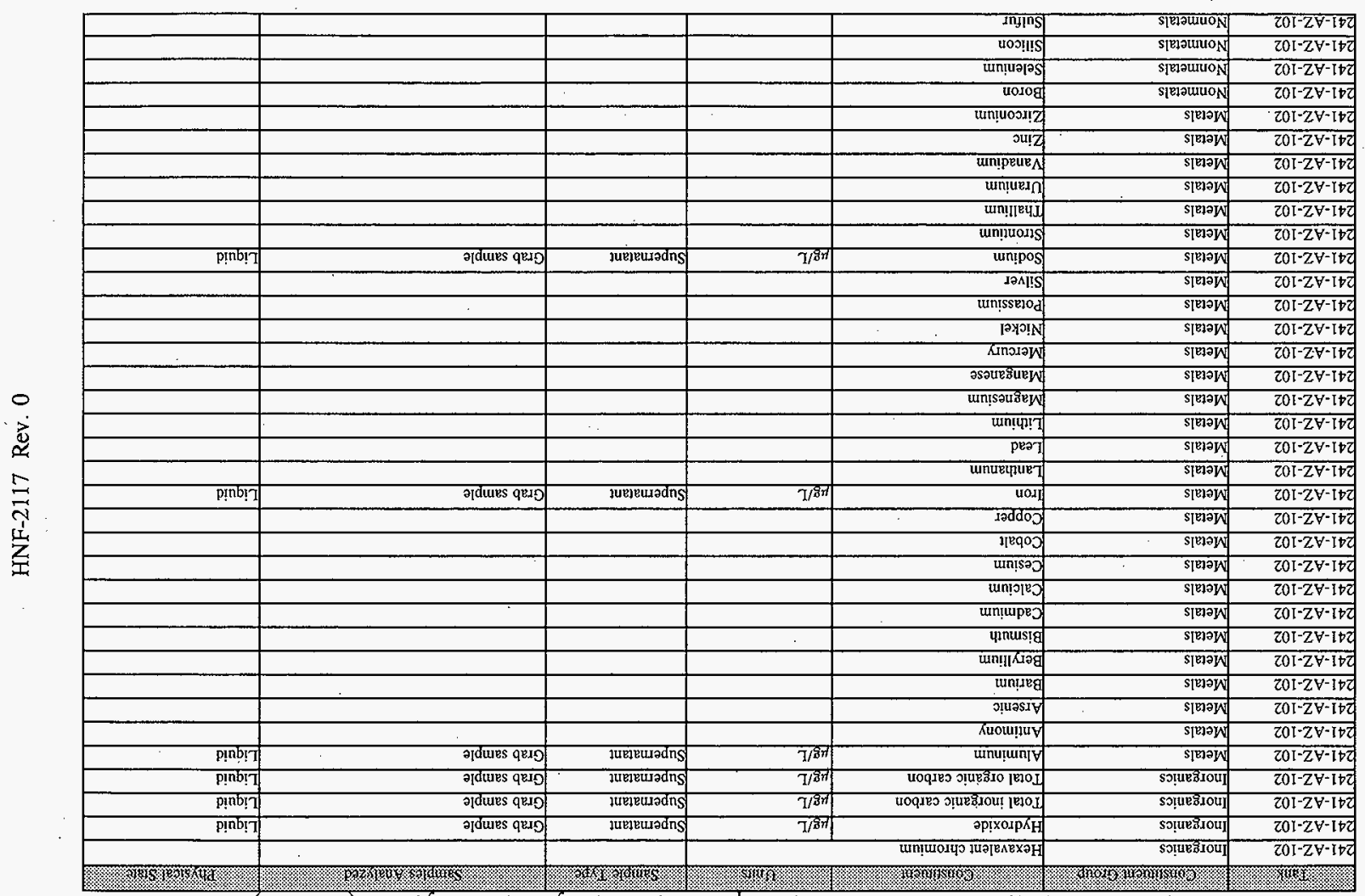

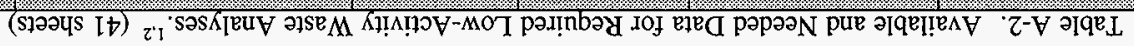


Table A-2. Available and Needed Data for Required Low-Activity Waste Analyses. ${ }^{1,2}$ (41 sheets)

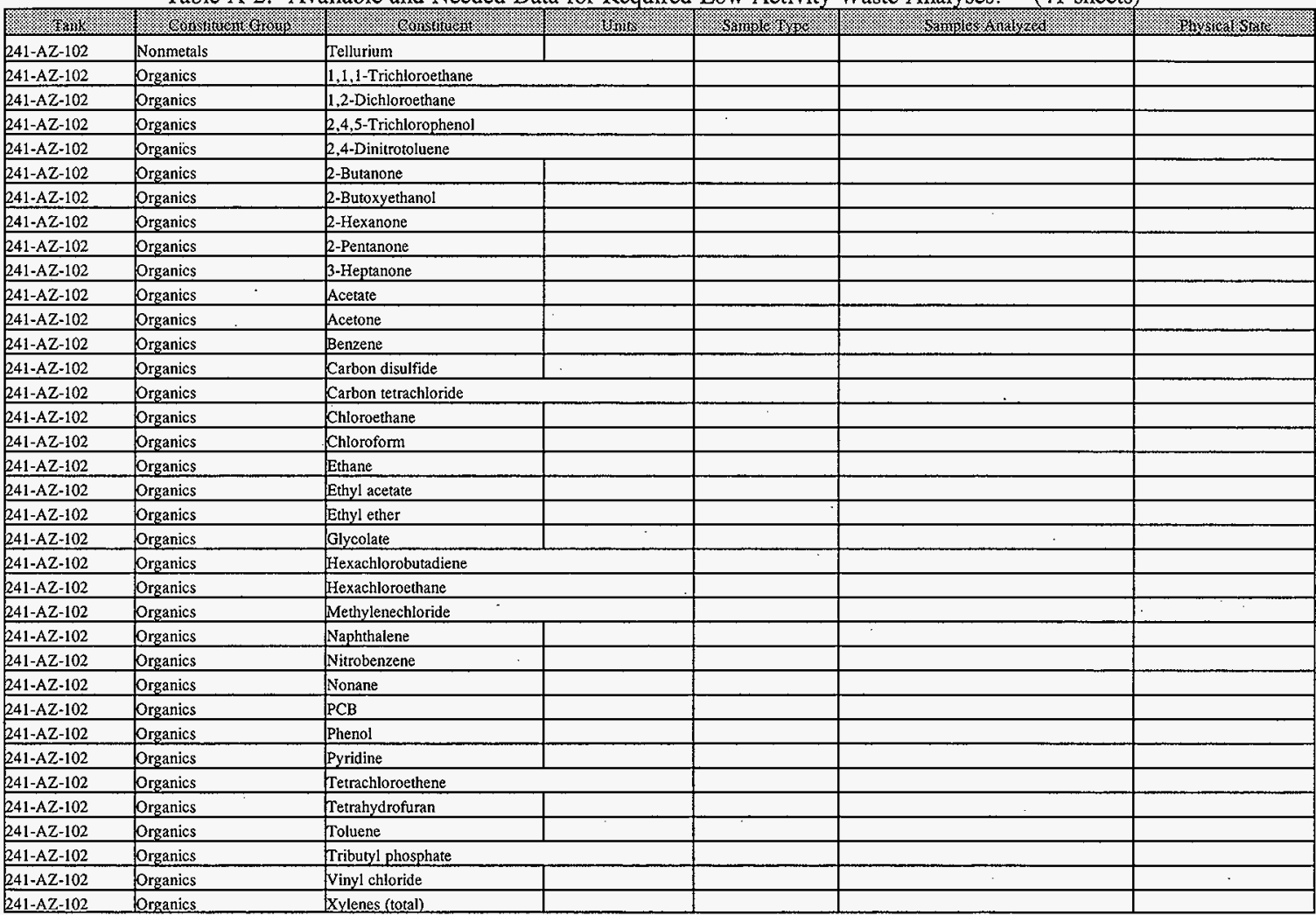




\begin{tabular}{|c|c|c|c|c|c|c|}
\hline & & & & uninuointa & sapt!mutionpey & $201-Z \forall-1+Z$ \\
\hline & . & & & $8 \varepsilon \tau-$-un!wounld & sop!̣nuoppey & $20 I-Z V-I+2$ \\
\hline p?तnb!n & गावuाes quexg & 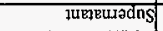 & $7 / ! \partial^{n}$ & 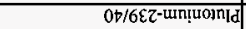 & sap!lonuoipey & $201-Z V-I \nabla Z$ \\
\hline & & & & 6£z-யn!̣uomn|d & sap!|juolpey & $201-Z V-I+Z$ \\
\hline pinb? & गेdues qEDO & IUEjewridns: & $7 / ! 0^{n}$ & $8 \varepsilon z$-un!ưoinld & sap!̣|jntio!pey & $\overline{Z 0 T-Z \forall-I t \gamma)}$ \\
\hline & & & & LEz-un!̣unıdəN & sap!̣|anuoppey & $20 t-Z V-1+2$ \\
\hline & & & & 6ZI-әи!poI & səp!̣|pnuoipey & $20 \pi-Z \forall-I \nabla y)$ \\
\hline & & & & maq ssong & sap!̣onuo!pry & $20 I-Z V-1+2$ \\
\hline & & & & exdie ssodg & səp!̣ponuo!pey & $20 I-Z \forall-I+2$ \\
\hline & & & & SSI-unṇdorng & Sap!̣|juolpey & $20 \pi-Z V^{*} I+2$ \\
\hline & & & & SSL/tSI-Uunịdoun司 & sәp!़|pnuo!pty & $20 T^{-Z H-I+Z}$ \\
\hline & & & & $\nabla \mathcal{S}$ [-tunțdorng & sap!̣anuoipey & $20 t^{-Z Z W-[\nabla]}$ \\
\hline & & & & 2SI-tunțdornat & Sap!|pnuoppey & $201-Z V \cdot I+Z$ \\
\hline & & & & 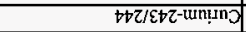 & Sap!̣jonuolpey & $20 I-Z \forall-I \circ Z$ \\
\hline & & & & ztz-un!unכ & sap!̣pnuo!pey & $20 I-Z V-I+Z$ \\
\hline & & & & 09-नाष्प & sap!̣pnuo!pey & $20[-Z V \cdot[+7]$ \\
\hline p!nb!i & Oldures qRD & luplewadns & $7 / ! \supset^{n}$ & LEI-tun!sa & sap!̣pnuọpley & $201-Z V-1+2$ \\
\hline & & & & 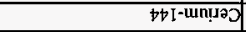 & sap!|pnuo!pey & $20 I-Z V-I+Z$ \\
\hline & & & & D[-troques & sap!̣ntoipty & $20 \tau-Z \forall-I+Z$ \\
\hline & & & & 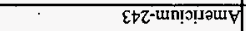 & sap!̣onuo!pey & $\overline{20 T \cdot Z V \cdot I+Z}$ \\
\hline p!nb!n & काdues quip & Ituetewadns & T/!! & Itz-un!ọ!autv| & sap!lantoippry & $20 !-Z V-I+\theta$ \\
\hline & & & & 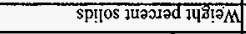 & 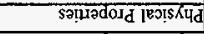 & $20 I^{-} \mathrm{ZV}-I+2$ \\
\hline & & & & 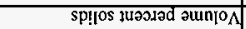 & 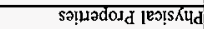 & $20 !-Z V-I \Delta Z$ \\
\hline & & & & 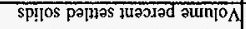 & 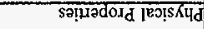 & $20 I-Z V-I \nabla z$ \\
\hline & & & & Ki!soss! & 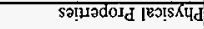 & $20[-Z \forall-I+t]$ \\
\hline & & SOVS & & 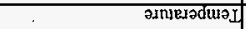 & 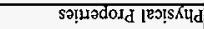 & $20 I-Z V-I+Z$ \\
\hline p!nb!r? & शpdures queng & 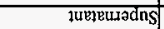 & ssa|?!un & Ri!nen गj!jads & 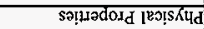 & $Z 0 !-Z \forall-I t Z$ \\
\hline & & & & aun & 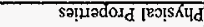 & $20 I-Z V-I \oplus Z$ \\
\hline & & & & 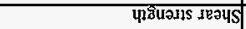 & 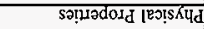 & $20[-Z \forall-[t]$ \\
\hline & & & & SPIIOS Pallies & 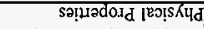 & $201-Z V-1+2$ \\
\hline pi़b!़ा & गdues quero & Juejeuradns & ssoplțun & 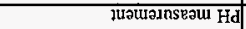 & 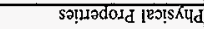 & $20 \mathrm{I}-Z \forall-I+Z$ \\
\hline pi़blin & गेdures qurg & ILधाEuadns & $\%$ & 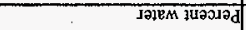 & 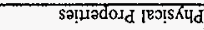 & $201-Z Y-1+2$ \\
\hline & & & & 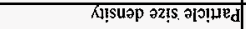 & 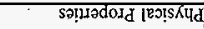 & $20[-Z \forall-I \nabla z$ \\
\hline p!חbi!n & श्वdures qua & IUEjEuradns & $\%$ & 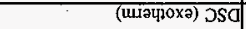 & 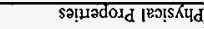 & $20 t-Z \forall-I \nabla Z$ \\
\hline & & & & 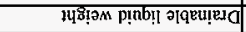 & 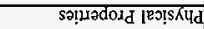 & $20 I-Z \forall-I+z$ \\
\hline & & & & Ka!surag & 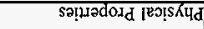 & $201-Z Y-1+2$ \\
\hline SWF & 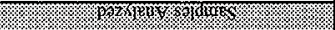 & 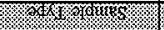 & & 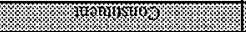 & 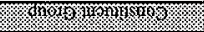 & 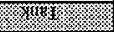 \\
\hline
\end{tabular}

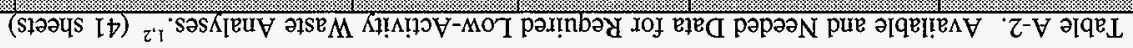


Table A-2. Available and Needed Data for Required Low-Activity Waste Analyses. ${ }^{1,2}$ (41 sheets)

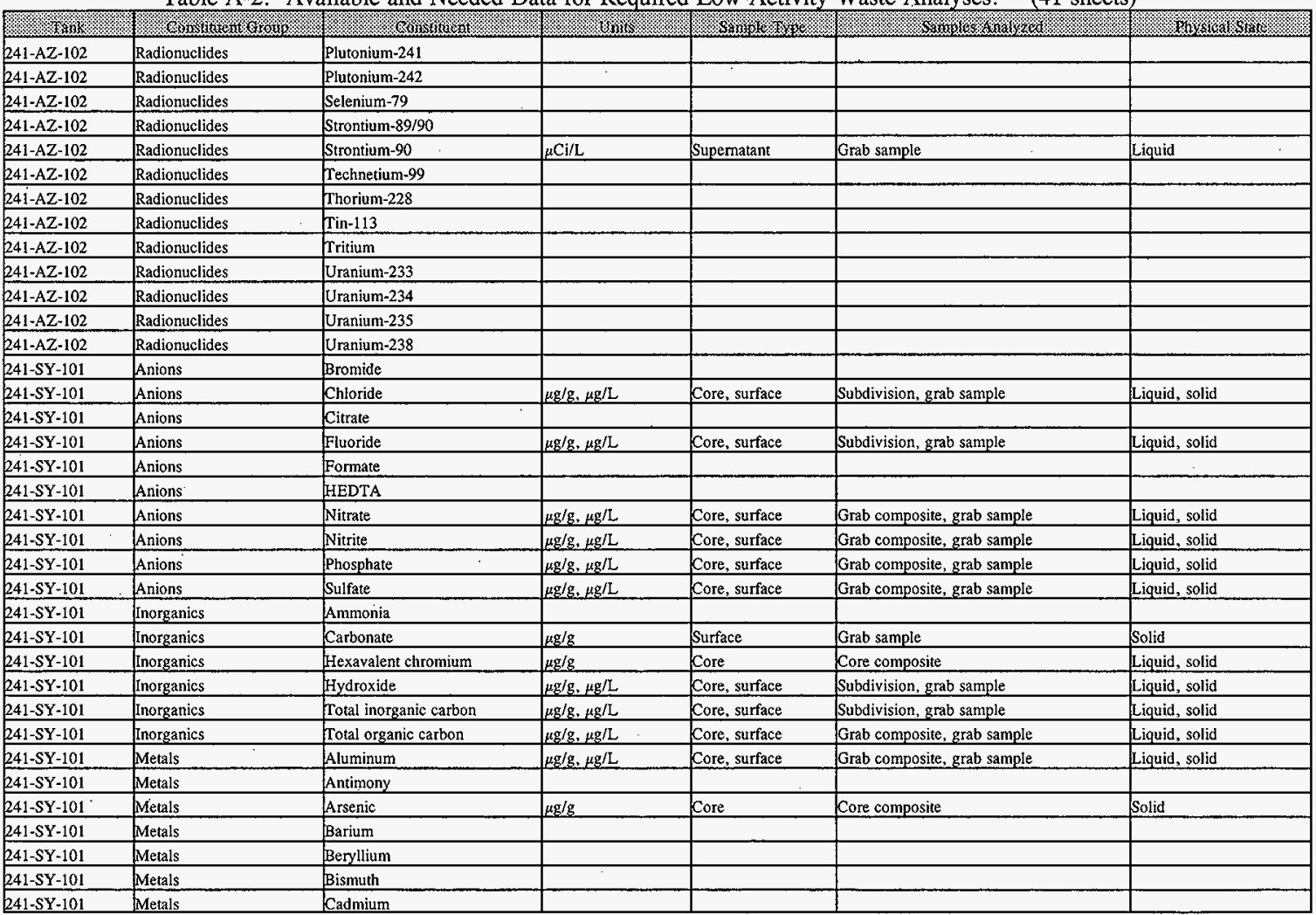


Table A-2. Available and Needed Data for Required Low-Activity Waste Analyses. ${ }^{1,2}$ (41 sheets)

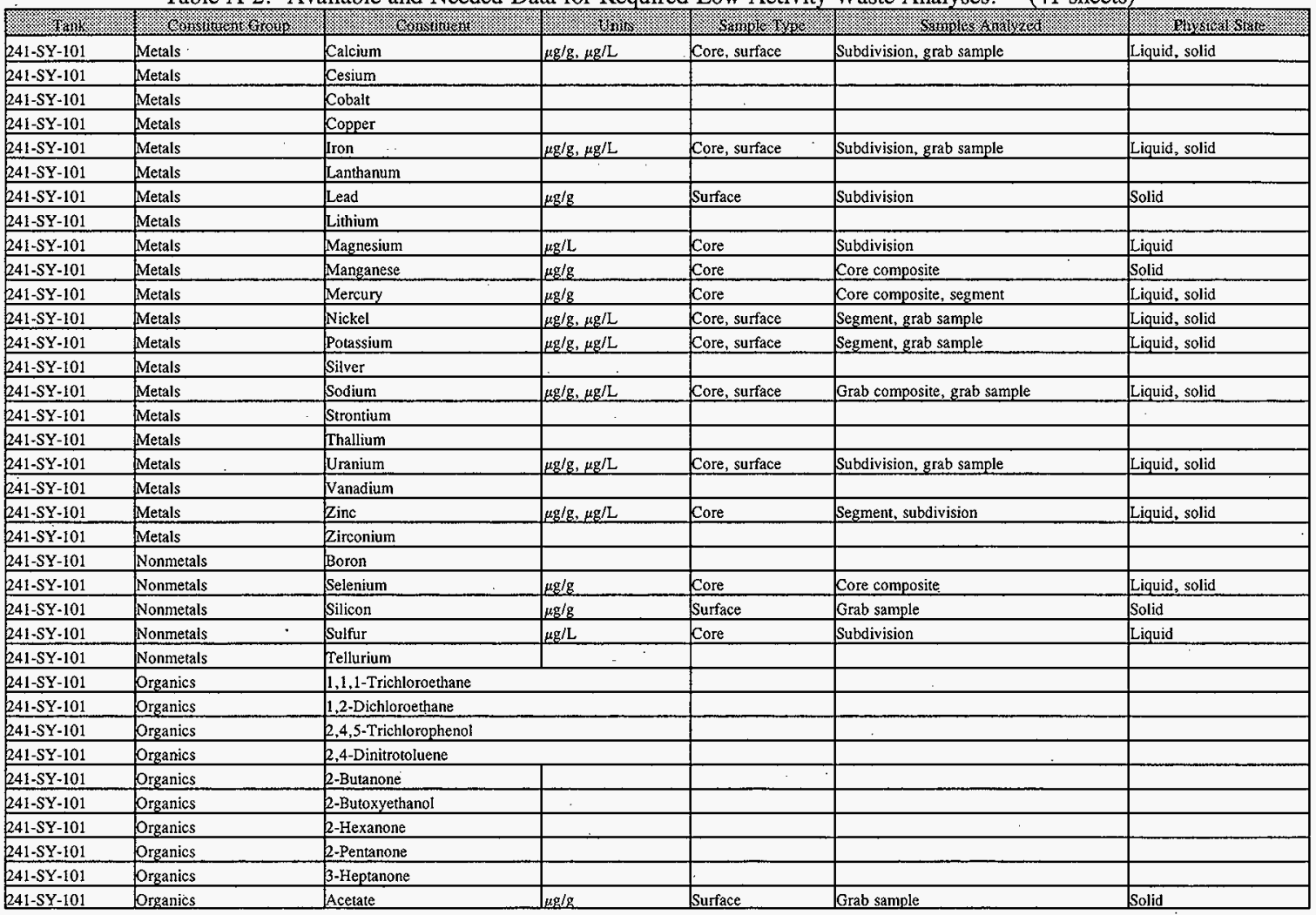


Table A-2. Available and Needed Data for Required Low-Activity Waste Analyses. ${ }^{1,2}$ (41 sheets)

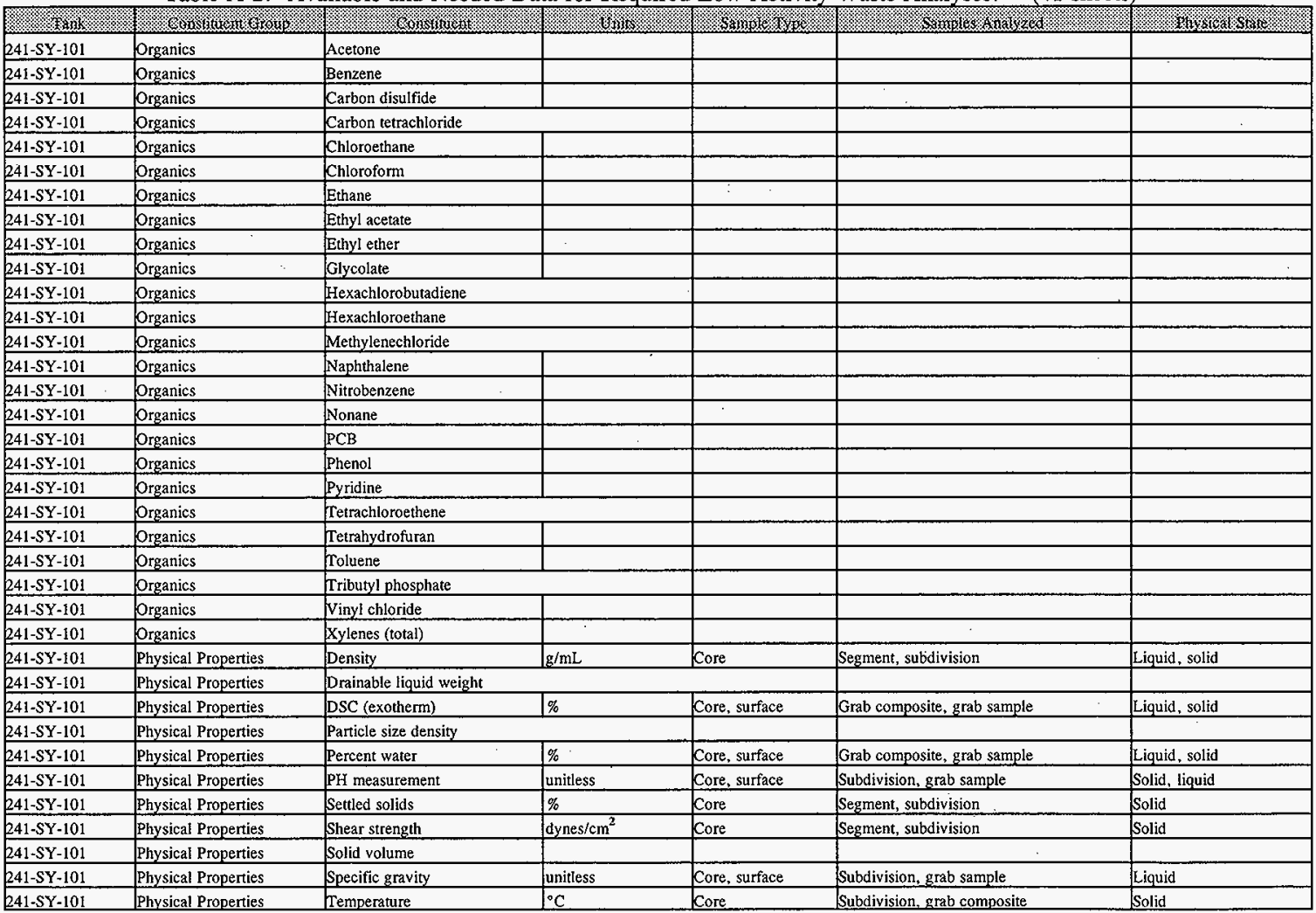


Table A-2. Available and Needed Data for Required Low-Activity Waste Analyses. ${ }^{1,2}$ (41 sheets)

\begin{tabular}{|c|c|c|c|c|c|c|}
\hline 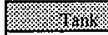 & W & (x) & 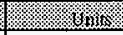 & 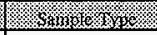 & 3. & 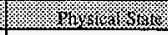 \\
\hline $241-5 Y-101$ & Physical Properties & Viscosity & $\mathrm{cP}$ & Core & Segment & Solid \\
\hline $241-S Y-101$ & Physical Properties & Volume percent settled solids & $\%$ & Core & Segment & Solid \\
\hline 241-SY-101 & Physical Properties & Volume percent solids & & & & \\
\hline $241-S Y-101$ & Physical Properties & Weight percent solids & $\%$ & Core & Subdivision, grab composite & Solid \\
\hline $241-\mathrm{SY}-101$ & Radionuclides & Americium-241 & $\mu \mathrm{Ci} / \mathrm{g}, \mu \mathrm{Ci} / \mathrm{L}$ & Core, surface & Subdivision, grab sample & Liquid, solid \\
\hline $241-S Y-101$ & Radionuclides & Americium-243 & & & & \\
\hline $241-S Y-101$ & Radionuclides & Carbon-14 & $\mu \mathrm{Ci} / \mathrm{g}$ & Core & Core composite & Solid \\
\hline 241-SY-101 & Radionuclides & Cerium-144 & & & & \\
\hline $241-\mathrm{SY}-101$ & Radionuclides & Cesium-137 & $\mu \mathrm{Ci} / \mathrm{g}, \mu \mathrm{Ci} / \mathrm{L}$ & Core, surface & Subdivision, grab sample & Liquid, solid \\
\hline 241-SY-101 & Radionuclides & Cobalt-60 & $\mu \mathrm{Ci} / \mathrm{g}$ & Surface & Grab sample & Solid \\
\hline $241-\mathrm{SY}-101$ & Radionuclides & Curium-242 & & & & \\
\hline $241-S Y-101$ & Radionuclides & Curium-243/244 & & & & \\
\hline $241-S Y-101$ & Radionuclides & Europium-152 & & & & \\
\hline 241-SY-101 & Radionuclides & Europium-154 & & & & \\
\hline $241-S Y-101$ & Radionuclides & Europium-154/155 & & & & \\
\hline 241-SY-101 & Radionuclides & Europium-155 & & & & \\
\hline 241-SY-101 & Radionuclides & Gross alpha & $\mu \mathrm{Ci} / \mathrm{g}, \mu \mathrm{Ci} / \mathrm{L}$ & Core, surface & Subdivision, grab sample & Liquid, solid \\
\hline $241-S Y-101$ & Radionuclides & Gross beta & $\mu \mathrm{Ci} / \mathrm{g}, \mu \mathrm{Ci} / \mathrm{L}$ & Core, surface & Subdivision, grab sample & Liquid, solid \\
\hline 241-SY-101 & Radionuclides & Iodine-129 & $\mu \mathrm{Ci} / \mathrm{g}, \mu \mathrm{Ci} / \mathrm{L}$ & Core, surface & Subdivision, grab sample & Liquid, solid \\
\hline 241-SY-101 & Radionuclides & Neptunium-237 & $\mu \mathrm{Ci} / \mathrm{g}, \mu \mathrm{Ci} / \mathrm{L}$ & Core, surface & Subdivision, grab sample & Liquid, solid \\
\hline 241-SY-101 & Radionuclides & Plutonium-238 & & & & \\
\hline 241-SY-101 & Radionuclides & Plutonium-239 & & & & \\
\hline 241-SY-101 & Radionuclides & Plutonium-239/40 & $\mu \mathrm{Ci} / \mathrm{g}, \mu \mathrm{Ci} / \mathrm{L}$ & Core, surface & Subdivision. grab sample & Liquid, solid \\
\hline 241-SY-101 & Radionuclides & Plutoium-240 & & & & \\
\hline $241-S Y-101$ & Radionuclides & Plutonium-241 & & & & \\
\hline $241-S Y-101$ & Radionuclides & Plutonium-242 & & & & \\
\hline $241-S Y-101$ & Radionuclides & Selenium-79 & & & & \\
\hline $241-S Y-101$ & Radionuclides & Strontium- $89 / 90$ & & & & \\
\hline 241-SY-101 & Radionuclides & Strontium-90 & $\mu \mathrm{Ci} / g, \mu \mathrm{Ci} / \mathrm{L}$ & Core, surface & Subdivision, grab sample & Liquid, solid \\
\hline $241-S Y-101$ & Radionuclides & Technetium-99 & $\mu \mathrm{Ci} / \mathrm{g}, \mu \mathrm{Ci} / \mathrm{L}$ & Core, surface & Subdivision, grab sample & Liquid, solid \\
\hline $241-S Y-101$ & Radionuclides & Thorium-228 & & & & \\
\hline $241-S Y-101$ & Radionuclides & $\operatorname{Tin}-113$ & & & & \\
\hline 241-SY-101 & Radionuclides & Tritium & & & & \\
\hline 241-SY-101 & Radionuclides & Uranium-233 & & & & \\
\hline 241-SY-101 & Radionuclides & Uranium-234 & & & & \\
\hline $241-S Y-101$ & Radionuclides & Uranium-235 & & & & \\
\hline
\end{tabular}


Table A-2. Available and Needed Data for Required Low-Activity Waste Analyses. ${ }^{1,2}$ (41 sheets)

\begin{tabular}{|c|c|c|c|c|c|c|}
\hline 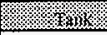 & Work & 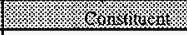 & Why & 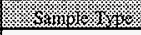 & S & 箖筑 \\
\hline 241-SY-101 & Radionuclides & Uranium-238 & & & & \\
\hline 241-SY-103 & Anions & Bromide & $\mu \mathrm{g} / \mathrm{g}, \mu \mathrm{g} / \mathrm{L}$ & Core & Subdivision, segment & Solid, liquid \\
\hline $241-S Y-103$ & Anions & Chloride & $\mu \mathrm{g} / \mathrm{g}, \mu \mathrm{g} / \mathrm{L}$ & Core & Core composite, segment & Solid, liquid \\
\hline $241-5 Y-103$ & Anions & Citrate & & & & \\
\hline 241-SY-103 & Anions & Fluoride & $\mu \mathrm{g} / \mathrm{g}, \mu \mathrm{g} / \mathrm{L}$ & Core & Core composite, segment & Solid, liquid \\
\hline $241-S Y-103$ & Anions & Formate & $\mu \mathrm{g} / \mathrm{g}, \mu \mathrm{g} / \mathrm{L}$ & Core & Core composite, segment & Solid, liquid \\
\hline 241-SY-103 & Anions & HEDTA & & & & \\
\hline $241-S Y-103$ & Anions & Nitrate & $\mu \mathrm{g} / \mathrm{g}, \mu \mathrm{g} / \mathrm{L}$ & Core & Core composite, segment & Solid, liquid \\
\hline $241-S Y-103$ & Anions & Nitrite & $\mu \mathrm{g} / \mathrm{g}, \mu \mathrm{g} / \mathrm{L}$ & Core & Core composite, segment & Solid, liquid \\
\hline $241-S Y-103$ & Anions & Phosphate & $\mu \mathrm{g} / \mathrm{g}, \mu \mathrm{g} / \mathrm{L}$ & Core & Core composite, segment & Solid, liquid \\
\hline $241-S Y-103$ & Anions & Sulfate & $\mu \mathrm{g} / \mathrm{g}, \mu \mathrm{g} / \mathrm{L}$ & Core & Core composite, segment & Solid, liquid \\
\hline $241-S Y-103$ & Inorganics & Ammonia & & 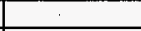 & & \\
\hline $241-S Y-103$ & Inorganics & Carbonate. & & & & \\
\hline $241-S Y-103$ & Inorganics & Hexavalent chromium & $\mu \mathrm{g} / \mathrm{g}, \mu \mathrm{g} / \mathrm{L}$ & Core & Core composite, segment & Solid, liquid \\
\hline 241-SY-103 & Inorganics & Hydroxide & $\mu \mathrm{g} / \mathrm{g}, \mu \mathrm{g} / \mathrm{L}$ & Core & Core composite, segment & Solid, liquid \\
\hline $241-S Y-103$ & Inorganics & Total inorganic carbon & $\mu g / g$ & Core, surface & Subdivision, grab composite & Solid \\
\hline $241-S Y-103$ & Inorganics & Total organic carbon & $\mu \mathrm{g} / \mathrm{g}, \mu \mathrm{g} / \mathrm{L}$ & Core, surface & Grab composite, segment & Solid, liquid \\
\hline 241-SY-103 & Metals & Aluminum & $\mu \mathrm{g} / \mathrm{g}, \mu \mathrm{g} / \mathrm{L}$ & Core & Subdivision, segment & Solid, liquid \\
\hline 241-SY-103 & Metals & Antimony & & & & \\
\hline $241-S Y-103$ & Metals & Arsenic & & & & \\
\hline 241-SY-103 & Metals & Barium & $\mu \mathrm{g} / \mathrm{L}$ & Core & Core composite, segment & Liquid \\
\hline 241-SY-103 & Metals & Beryllium & & & & \\
\hline 241-SY-103 & Metals & Bismuth & $\mu \mathrm{g} / \mathrm{L}$ & Core & Core composite, segment & Liquid \\
\hline $241-S Y-103$ & Metals & Cadmium & & & & \\
\hline 241-SY-103 & Metals & Calcium & $\mu \mathrm{g} / \mathrm{g}, \mu \mathrm{g} / \mathrm{L}$ & Core & Subdivision, segment & Solid, liquid \\
\hline $241-S Y-103$ & Metals & Cesium & & & & \\
\hline $241-S Y-103$ & Metals & Cobalt & & & $\cdot$ & \\
\hline $241-S Y-103$ & Metals & Copper & & & & \\
\hline $241-S Y-103$ & Metals & Iron & $\mu \mathrm{g} / \mathrm{g}, \mu \mathrm{g} / \mathrm{L}$ & Core & Subdivision, segment & Solid, liquid \\
\hline $241-S Y-103$ & Metals & Lanthanum & & & & \\
\hline 241-SY-103 & Metals & Lead & & & & \\
\hline $241-S Y-103$ & Metals & Lithium & $\mu \mathrm{g} / \mathrm{g}, \mu \mathrm{g} / \mathrm{L}$ & Core & Subdivision, core composite & Solid, liquid \\
\hline 241-SY-103 & Metals & Magnesium & & & & \\
\hline $241-5 Y-103$ & Metals & Manganese & & & & \\
\hline 241-SY-103 & Metals & Mercury & & & & \\
\hline $241-S Y=103$ & Metals & Nickel & $\mu \mathrm{g} / \mathrm{g}, \mu \mathrm{g} / \mathrm{L}$ & Core & Subdivision, segment & Solid, liquid \\
\hline
\end{tabular}


Table A-2. Available and Needed Data for Required Low-Activity Waste Analyses, ${ }^{1,2}$ (41 sheets)

\begin{tabular}{|c|c|c|c|c|c|c|}
\hline Kris & 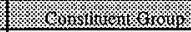 & 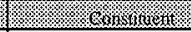 & 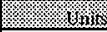 & 策 & 10. & 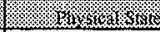 \\
\hline 241-SY-103 & Metals & Potassium & $\mu \mathrm{g} / \mathrm{g}, \mu \mathrm{g} / \mathrm{L}$ & Core & Subdivision, segment & Solid, liquid \\
\hline $241-S Y-103$ & Metals & Silver & & & & \\
\hline $241-S Y-103$ & Metals & Sodium & $\mu \mathrm{g} / \mathrm{g}, \mu \mathrm{g} / \mathrm{L}$ & Core & Subdivision, segment & Solid, liquid \\
\hline 241-SY-103 & Metals & Strontium & & - & & \\
\hline 241-SY-103 & Metals & Thallium & & & & \\
\hline 241-SY-103 & Metals & Uranium & $\mu \mathrm{g} / \mathrm{g}, \mu \mathrm{g} / \mathrm{L}$ & Core & Subdivision, segment & Solid, liquid \\
\hline $241-S Y-103$ & Metals & Vanadium & & & & \\
\hline 241-SY-103 & Metals & Zinc & $\mu \mathrm{g} / \mathrm{g}, \mu \mathrm{g} / \mathrm{L}$ & Core & Subdivision, segment & Solid, liquid \\
\hline 241-SY-103 & Metals & Zirconium & $\mu \mathrm{g} / \mathrm{g}, \mu \mathrm{g} / \mathrm{L}$ & Core & Subdivision, segment & Solid, liquid \\
\hline 241-SY-103 & Nonmetals & Boron & $\mu \mathrm{g} / \mathrm{L}$ & Core & Core composite, segment & Liquid \\
\hline 241-SY-103 & Nonmetals & Selenium & & & & \\
\hline 241-SY-103 & Nonmetals & Silicon & $\mu \mathrm{g} / \mathrm{L}$ & Core & Core composite, segment & Liquid \\
\hline 241-SY-103 & Nonmetals & Sulfur & & & & \\
\hline 241-SY-103 & Nonmetals & Tellurium & & & & \\
\hline 241-SY-103 & Organics & 1,1,1-Trichloroethane & & & & \\
\hline $241-S Y-103$ & Organics & 1,2-Dichloroethàne & & & & \\
\hline $241-S Y-103$ & Organics & 2,4,5-Trichlorophenol & & & & \\
\hline 241-SY-103 & Organics & 2,4-Dinitrotoluene & & & & \\
\hline $241-S Y-103$ & Organics & 2-Butanone & & & & \\
\hline $241-S Y-103$ & Organics & 2-Butoxyethanol & & & & \\
\hline 241-SY-103 & Organics & 2-Hexanone & & & & \\
\hline 241-SY-103 & Organics & 2-Pentanone & & . & & \\
\hline $241-S Y-103$ & Organics & 3-Heptanone & & & & \\
\hline 241-SY-103 & Organics & Acetate & $\mu \mathrm{g} / \mathrm{g}, \mu \mathrm{g} / \mathrm{L}$ & Core & Core composite, segment & Solid, liquid \\
\hline $241-S Y \cdot 103$ & Organics & Acetone & & & & \\
\hline $241-S Y-103$ & Organics & Benzene & & & & \\
\hline 241-SY-103 & Organics & Carbon disulfide & & & & \\
\hline $241-S Y-103$ & Organics & Carbon tetrachloride & & & & \\
\hline $241-S Y-103$ & Organics & Chloroethane & & & & \\
\hline 241-SY-103 & Organics & Chloroform & & & & \\
\hline $241-S Y-103$ & Organics & Ethane & & & & \\
\hline $241-S Y-103$ & Organics & Ethyl acetate & & & & \\
\hline $241-S Y-103$ & Organics & Ethyl ether & & & & \\
\hline $241-S Y-103$ & Organics & Glycolate & & & & \\
\hline $241-S Y-103$ & Organics & Hexachlorobutadiene & & & & \\
\hline 241-SY.103 & Organics & Hexachloroethane & & & & \\
\hline
\end{tabular}




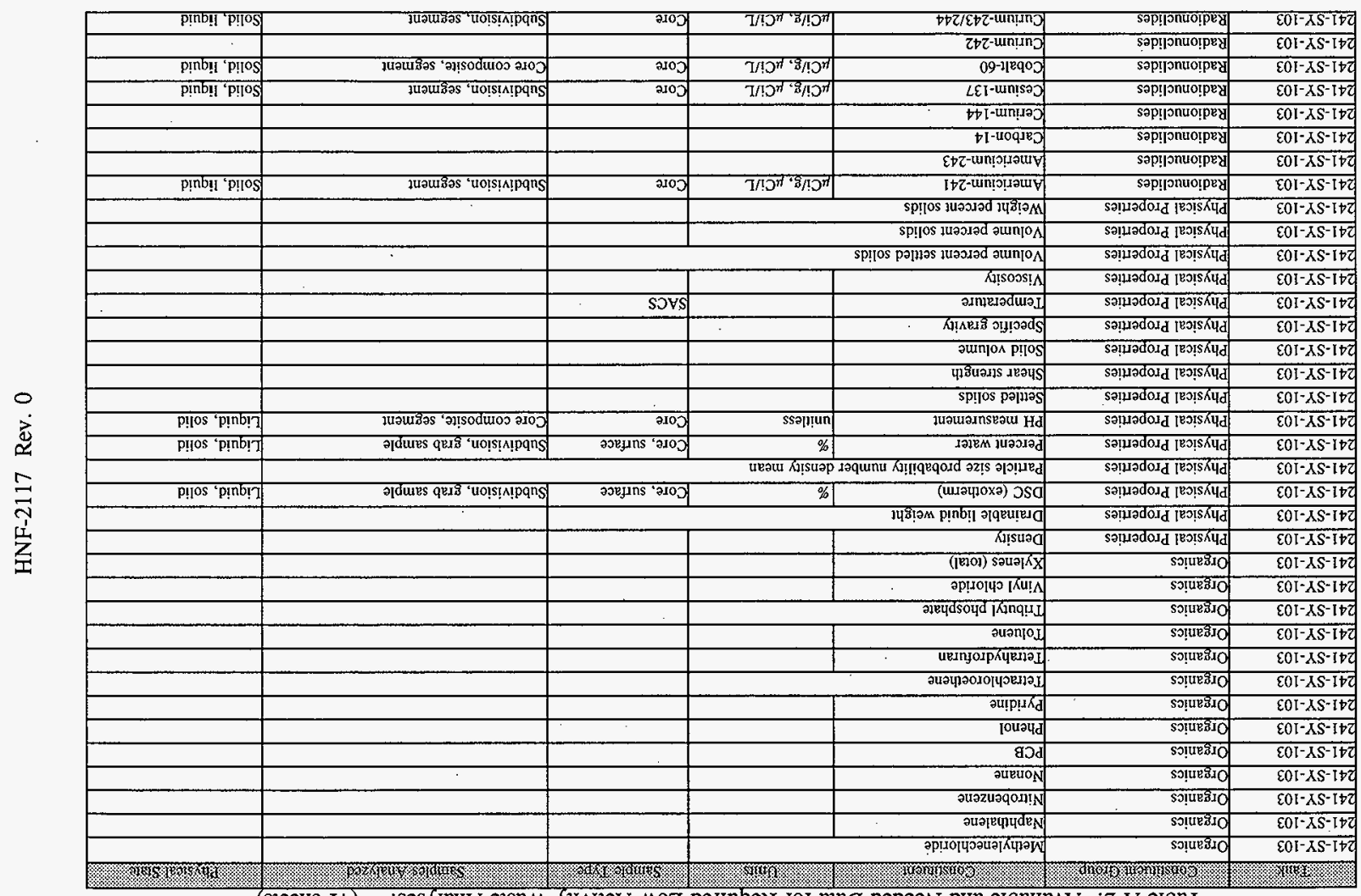

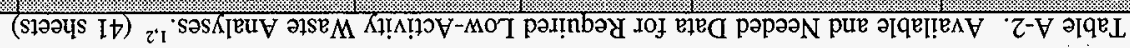


Table A-2. Available and Needed Data for Required Low-Activity Waste Analyses. ${ }^{1.2}$ (41 sheets)

\begin{tabular}{|c|c|c|c|c|c|c|}
\hline 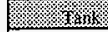 & 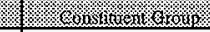 & 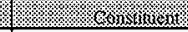 & W/6.6. & \% & 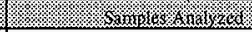 & 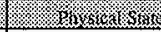 \\
\hline $241-S Y-103$ & Radionuclides & Europium-152 & & & & \\
\hline $241-S Y-103$ & Radionuclides & Europium-154 & $\mu \mathrm{Ci} / \mathrm{g}, \mu \mathrm{Ci} / \mathrm{L}$ & Core & Core composite, segment & Solid, liquid \\
\hline $241-S Y-103$ & Radionuclides & Europium-154/155 & & & & \\
\hline $241-S Y-103$ & Radionuclides & Europium-155 & $\mu \mathrm{Ci} / \mathrm{g}, \mu \mathrm{Ci} / \mathrm{L}$ & Core & Core composite, segment & Solid, liquid \\
\hline $241-S Y-103$ & Radionuclides & Gross alpha & $\mu \mathrm{Ci} / \mathrm{g}, \mu \mathrm{Ci} / \mathrm{L}$ & Core & Subdivision, segment & Solid, liquid \\
\hline 241-SY-103 & Radionuclides & Gross beta & $\mu \mathrm{Ci} / \mathrm{g}, \mu \mathrm{Ci} / \mathrm{L}$ & Core & Subdivision, segment & Solid, liquid \\
\hline 241-SY-103 & Radionuclides & Iodine-129 & $\mu \mathrm{Ci} / \mathrm{g}, \mu \mathrm{Ci} / \mathrm{L}$ & Core & Subdivision, segment & Solid, liquid \\
\hline $241-S Y-103$ & Radionuclides & Neptunium-237 & $\mu \mathrm{Ci} / \mathrm{g}, \mu \mathrm{Ci} / \mathrm{L}$ & Core & Subdivision, segment & Solid, liquid \\
\hline $241-S Y-103$ & Radionuclides & Plutonium-238 & $\mu \mathrm{Ci} / \mathrm{g}, \mu \mathrm{Ci} / \mathrm{L}$ & Core & Subdivision, segment & Solid, liquid \\
\hline $241-S Y-103$ & Radionuclides & Plutonium-239 & & & & \\
\hline $241-S Y-103$ & Radionuclides & Plutonium-239/40 & $\mu \mathrm{Ci} / \mathrm{g}, \mu \mathrm{Ci} / \mathrm{L}$ & Core & Subdivision, segment & Solid, liquid \\
\hline $241-S Y-103$ & Radionuclides & Plutonium-238 & & & & \\
\hline $241-S Y-103$ & Radionuclides & Plutonium-240 & & & & \\
\hline $241-S Y-103$ & Radionuclides & Plutonium-241 & & & & \\
\hline $241-S Y-103$ & Radionuclides & Plutonium-242 & & & & \\
\hline $241-S Y-103$ & Radionuclides & Selenium-79 & & & & \\
\hline $241-S Y-103$ & Radionuclides & Strontium-89/90 & $\mu \mathrm{Ci} / \mathrm{g}, \mu \mathrm{Ci} / \mathrm{L}$ & Core & Subdivision, segment & Solid, liquid \\
\hline $24 !-S Y-103$ & Radionuclides & Strontium-90 & & & & \\
\hline $241-5 Y-103$ & Radionuclides & Technetium-99 & $\mu \mathrm{Ci} / \mathrm{g}, \mu \mathrm{Ci} / \mathrm{L}$ & Core & Subdivision, segment & Solid, liquid \\
\hline $241-S Y-103$ & Radionuclides & Thorium-228 & & & & \\
\hline $241-S Y-103$ & Radionuclides & $\operatorname{Tin}-113$ & & & & \\
\hline $241-S Y-103$ & Radionuclides & Tritium & $\mu \mathrm{Ci} / \mathrm{g}, \mu \mathrm{Ci} / \mathrm{L}$ & Core & Core composite, segment & Solid, liquid \\
\hline $241-S Y-103$ & Radionuclides & Uranium-233 & & & & \\
\hline $241-S Y-103$ & Radionuclides & Uranium-234 & & & & \\
\hline $241-S Y \cdot 103$ & Radionuclides & Uranium-235 & & & & \\
\hline $241-S Y-103$ & Radionuclides & Uranium-238 & & & & \\
\hline
\end{tabular}

Notes:

'Requirements and available data are shown for each LAW and HLW tank in scope. No data is available where colums, 4, 5, 6, and 7 are blank

${ }^{2}$ Analytes listed in column 3 are those included in the Tank Characterization Database. This table does not include requirements not listed in TCD.

$\begin{array}{llllll}\mathrm{SACS} & = & \text { Surveillance Analysis Computer System } & \mu \mathrm{G} / \mathrm{mL} & = & \text { micrograms per milliter } \\ \mathrm{cm}^{2} & = & \text { square centimeters } & \mu \mathrm{g} / \mathrm{g} & = & \text { micrograms per gram } \\ \mathrm{J} / \mathrm{g} & = & \text { joules per gram } & \mu \mathrm{g} / \mathrm{L} & = & \text { micorgrams per liter } \\ \mu \mathrm{Ci} / \mathrm{L} & = & \text { micorcuries per liter } & \% & = & \text { percent }\end{array}$


Table A-3. Available and Needed Data for High-Level Waste Privatization Requirements. ${ }^{1.2}$ (13 sheets)

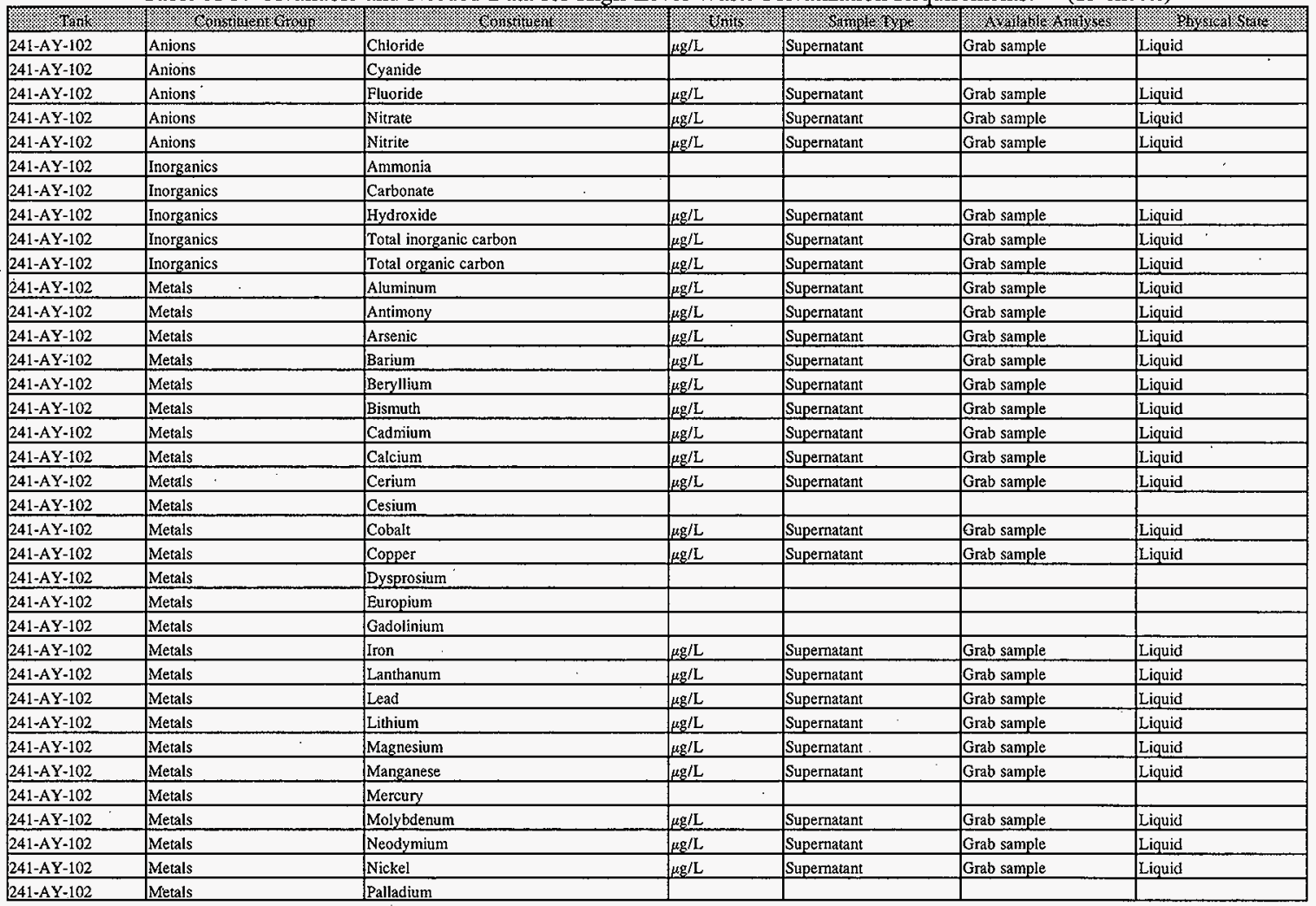


Table A-3. Available and Needed Data for High-Level Waste Privatization Requirements. ${ }^{1,2}$ (13 sheets)

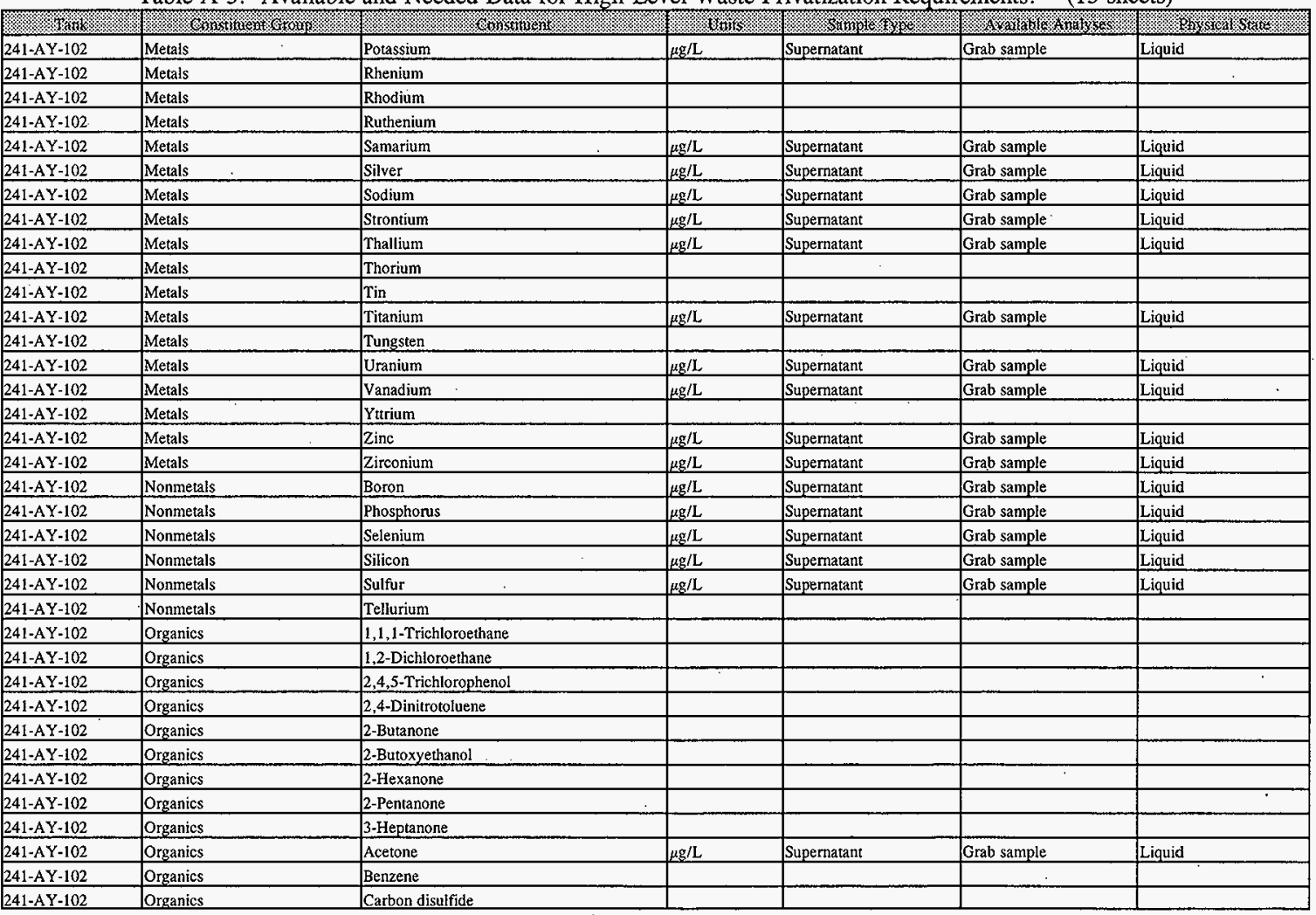


Table A-3. Available and Needed Data for High-Level Waste Privatization Requirements. ${ }^{1.2}$ (13 sheets)

\begin{tabular}{|c|c|c|c|c|c|c|}
\hline Trixik & \% & (6) & 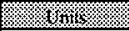 & \% & 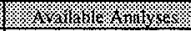 & 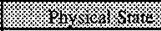 \\
\hline 241-AY-102 & Organics & Carbon tetrachloride & & & & \\
\hline $241-A Y-102$ & Organics & Chloroethane & & & & \\
\hline $241-\mathrm{AY}=102$ & Organics & Chloroform & & & & \\
\hline 241-AY-102 & Organics & Ethane & & & & \\
\hline 241-AY-102. & Organics & Ethyl acetate & & & & \\
\hline 241-AY-102 & Organics & Ethyl ether & & & & \\
\hline $241-\mathrm{AX}-102$ & Organics & Hexachlorobutadiene & & & & \\
\hline $241-\mathrm{AY}-102$ & Organics & Hexachloroethane & & & & \\
\hline $241-\mathrm{AY}-102$ & Organics & Methylenechlotide & & & & \\
\hline 241-AY-102 & Organics & Naphthalene & & & & \\
\hline 241-AY-102 & Organics & Nitrobenzene & & & & \\
\hline $241-\mathrm{AY}-102$ & Organics & Nonane & & & & \\
\hline 241-AY-102 & Organics & $\mathrm{PCB}$ & & & & \\
\hline 241-AY-102 & Organics & Phenol & & & & \\
\hline $241-\mathrm{AY}-102$ & Organics & Pyridine & & & & \\
\hline 241-AY-102 & Organics & Tetrachloroethene & & & & \\
\hline $241-\mathrm{AY}-102$ & Organics & Tetrahydrofuran & & & & \\
\hline 241-AY-102 & Organics & Toluene & & & & \\
\hline $241-\mathrm{AY}-102$ & Organics & Tributyl phosphate & & & & \\
\hline 241-AY-102 & Organics & Vinyl chloride & & & & \\
\hline 241-AY-102 & Organics & Xylenes (total) & & & & \\
\hline 241-AY-102 & Physical Properties & Density & & & & \\
\hline 241-AY-102 & Physical Properties & DSC (exotherm) & $\%$ & Supernatant & Grab sample & Liquid \\
\hline $241-\mathrm{AY}-102$ & Physical Properties & Particle size density & & & & \\
\hline 241-AY-102 & Physical Properties & Percent water & $\%$ & Supernatant & Grab sample & Liquid \\
\hline 241-AY-102 & Physical Properties & PH measurement & unitless & Supernatant & Grab sample & Liquid \\
\hline $241-A Y-102$ & Physical Properties & Settled solids & & & & \\
\hline $241-\mathrm{AY}-102$ & Physical Properties & Shear strength & & & & \\
\hline 241-AY-102 & Physical Properties & Solid density & & & & \\
\hline 241-AY-102 & Physical Properies & Specific gravity & unitless & Supernatant & Grab sample & Liquid \\
\hline 241-AY-102 & Physical Properties & Temperature & & SACS & & \\
\hline $241-A Y-102$ & Physical Properties & Total dissolved solids & & & & \\
\hline 241-AY-102 & Physical Properties & Viscosity & & & & . \\
\hline 241-AY-102 & Physical Properties & Weight percent oxides & & & & \\
\hline 241-AY-102 & Physical Properties & Weight percent solids & $\%$ & Supernatant & Grab sample & Liquid \\
\hline 241-AY-102 & Physical Properties & Yield point & & & & \\
\hline
\end{tabular}


Table A-3. Available and Needed Data for High-Level Waste Privatization Requirements..$^{2}{ }^{2}$ (13 sheets)

\begin{tabular}{|c|c|c|c|c|c|c|}
\hline 3hn & 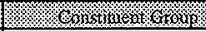 & 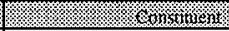 & 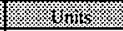 & 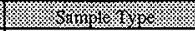 & 筑 & 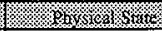 \\
\hline 241-AY-102 & Radionuclides & Americium-241 & $\mu \mathrm{Ci} / \mathrm{L}$ & Supernatant & Grab sample & Liquid \\
\hline 241-AY-102 & Radionuclides & Americium-243 & & & & \\
\hline $241-\mathrm{AY}-102$ & Radionuclides & Antimony-125 & & & & \\
\hline 241-AX-102 & Radionuclides & Carbon-14 & & & & \\
\hline 241-AY-102 & Radionuclides & Cerium-144 & & & & \\
\hline $241-\mathrm{A}, \mathrm{Y}-102$ & Radionuclides & Cerium/Praseodymium- 144 & & & & \\
\hline 241-AY-102 & Radionuclides & Cesium-134 & & & & \\
\hline 241-AY-102 & Radionuclides & Cesium-137 & $\mu \mathrm{Ci} / \mathrm{L}$ & Supernatant & Grab sample & Liquid \\
\hline 241-AY-102 & Radionuclides & Cobaitt- 60 & $\mu \mathrm{Ci} / \mathrm{L}$ & Supernatant & Grab sample & Liquid \\
\hline 241-AY-102 & Radionuclides & Curium-242 & & & & \\
\hline 241-AY-102 & Radionuclides & Curium-243/244 & & & & \\
\hline $241-\mathrm{AY}-102$ & Radionuclides & Curium-244 & & & & \\
\hline $241-\mathrm{AY}-102$ & Radionuclides & Europium-152 & & & & \\
\hline $24 !-A Y-102$ & Radionuctides & Europium-154 & & & & \\
\hline 241-AY-102 & Radionuclides & Europium-155 & & & & \\
\hline 241-AY -102 & Radionuclides & Gross alpha & & & & \\
\hline $241-\mathrm{AY}-102$ & Radionuclides & Gross beta & & & & \\
\hline 241-AY-102 & Radionuclides & Iodine-129 & & & & \\
\hline $241-\mathrm{AX}-102$ & Radionuclides & Neptunium-237 & & & & \\
\hline 241-AY-102 & Radionuclides & Nickel-59 & & & & \\
\hline 241-AY-102 & Radionuclides & Nickel-63 & & & & \\
\hline $241-\mathrm{AY}-102$ & Radionuclides & Plutonium-238 & & & & \\
\hline $24 \mathrm{t}-\mathrm{AY}-102$ & Radionuclides & Plutonium-239 & & & & \\
\hline 24I-AY-102 & Radionuclides & Plutonium-239/40 & $\mu \mathrm{Ci} / \mathrm{L}$ & Supernatant & Grab sample & Liquid \\
\hline $24 \mathrm{I}-\mathrm{AY}-102$ & Radionuclides & Plutonium-240 & & $\cdot$ & & \\
\hline 241-AY-102 & Radionuclides & Plutonim-241 & & & & \\
\hline $241-\mathrm{AY}-102$ & Radionuclides & Plutonium-242 & & & & \\
\hline 241-AY-102 & Radionuclides & Ruthenium-106 & & & & \\
\hline $24 \mathrm{I}-\mathrm{AY}-102$ & Radionuclides & Selenium-79 & & & & \\
\hline $24 \mathrm{~L}-\mathrm{AX}-102$ & Radionuclides & Strontium-90 & $\mu \mathrm{Ci} / \mathrm{L}$ & Supernatant & Grab sample & Liquid \\
\hline $241-\mathrm{AY}-102$ & Radionuclides & Technetium-99 & & & & \\
\hline $241-\mathrm{AY}-102$ & Radionuclides & Thorium-228 & & & & \\
\hline 241-AY-102 & Radionuclides & Tin-113 & & & & \\
\hline $241-\mathrm{AY}-102$ & Radionuclides & Total alpha, plutonium & & & & \\
\hline 241-AY-102 & Radiontclides & Tritium & & & & \\
\hline 241-AY-102 & Radionuclides & Uranium-236 & & & & \\
\hline
\end{tabular}


Table A-3. Available and Needed Data for High-Level Waste Privatization Requirements. ${ }^{1,2}$ (13 sheets)

\begin{tabular}{|c|c|c|c|c|c|c|}
\hline 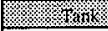 & 3\% & W & . & 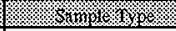 & 13. & 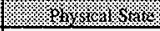 \\
\hline 241-AY-102 & Radionuclides & Uranium-233 & & & & \\
\hline 241-AY-102 & Radionuclides & Uranium-234 & & & & \\
\hline 241-AY-102 & Radionuclides & Uranium-235 & & & & \\
\hline $241-A Y-102$ & Radionuclides & Uranium-238 & & & & \\
\hline 241-AZ-101 & Anions & Chioride & $\mu \mathrm{g} / \mathrm{L}$ & Supernatant & Grab sample & Liquid \\
\hline 241-AZ-101. & Anions & Cyanide & & & & \\
\hline 241-AZ-101 & Anions & Fluoride & $\mu \mathrm{g} / \mathrm{L}$ & Supernatant & Grab sample & Liquid \\
\hline 241-AZ-101 & Anions & Nitrate & $\mu \mathrm{g} / \mathrm{L}$ & Supernatant & Grab sample & Liquid \\
\hline 241-AZ-101 & Anions & Nitrite & $\mu \mathrm{g} / \mathrm{L}$ & Supernatant & Grab sample & Liquid \\
\hline 241-AZ-101 & Inorganics & Ammonia & & & & \\
\hline 241-AZ-101 & Inorganics & Carbonate & & & & \\
\hline $241-\mathrm{AZ}-101$ & Inorganics & Hydroxide & $\mu \mathrm{g} / \mathrm{L}$ & Supernatant & Grab sample & Liquid \\
\hline 241-AZ-101. & Inorganics & Total inorganic carbon & $\mu \mathrm{g} / \mathrm{L}$ & Supernatant & Grab sample & Liquid \\
\hline 241-AZ-101 & Inorganics & Total organic carbon & $\mu \mathrm{g} / \mathrm{L}$ & Supernatant & Grab sample & Liquid \\
\hline 241-AZ-101 & Metals & Aluminum & $\mu \mathrm{g} / \mathrm{L}$ & Supernatant & Grab sample & Liquid \\
\hline $241-\mathrm{AZ}-101$ & Metals & Antimony & & & & \\
\hline 241-AZ-101 & Metals & Arsenic & & & & \\
\hline 241-AZ-101 & Metals & Barium & & & & \\
\hline $241-A Z-101$ & Metals & Beryllium & & & & \\
\hline 241-AZ-101 & Metals & Bismuth & & & & \\
\hline $241-\mathrm{AZ}-101$ & Metals & Cadmium & & & & \\
\hline 241-AZ-101 & Metals & Calcium & & & & \\
\hline $241-\mathrm{AZ}-101$ & Metals & Cerium & & & & \\
\hline 241-AZ-101 & Metals & Cesium & & & & \\
\hline $241-A Z-101$ & Metals & Cobalt & & & & \\
\hline $241-\mathrm{AZ}-101$ & Metals & Copper & & & & \\
\hline 241-AZ-101 & Metais & Dysprosium & & & & \\
\hline $241-\mathrm{AZ}-101$ & Metals & Europium & & & & \\
\hline 241-AZ-101 & Metals & Gadolinium & & & & \\
\hline $241-A Z-101$ & Metals & Iron & $\mu \mathrm{g} / \mathrm{L}$ & Supermatant & Grab sample & Liquid \\
\hline 241-AZ-101 & Metals & Lanthanum. & & & & \\
\hline $241-A Z-101$ & Metals & Lead & & & & \\
\hline 241-AZ-101 & Metals & Lithium & & & & \\
\hline $241-A Z-101$ & Metals & Magnesium & & & & \\
\hline 241-AZ-101 & Metals & Manganese & & & & \\
\hline $241-\mathrm{AZ}-101$ & Metals & Mercury & & & & \\
\hline
\end{tabular}


Table A-3. Available and Needed Data for High-Level Waste Privatization Requirements. ${ }^{1,2}$ (13 sheets)

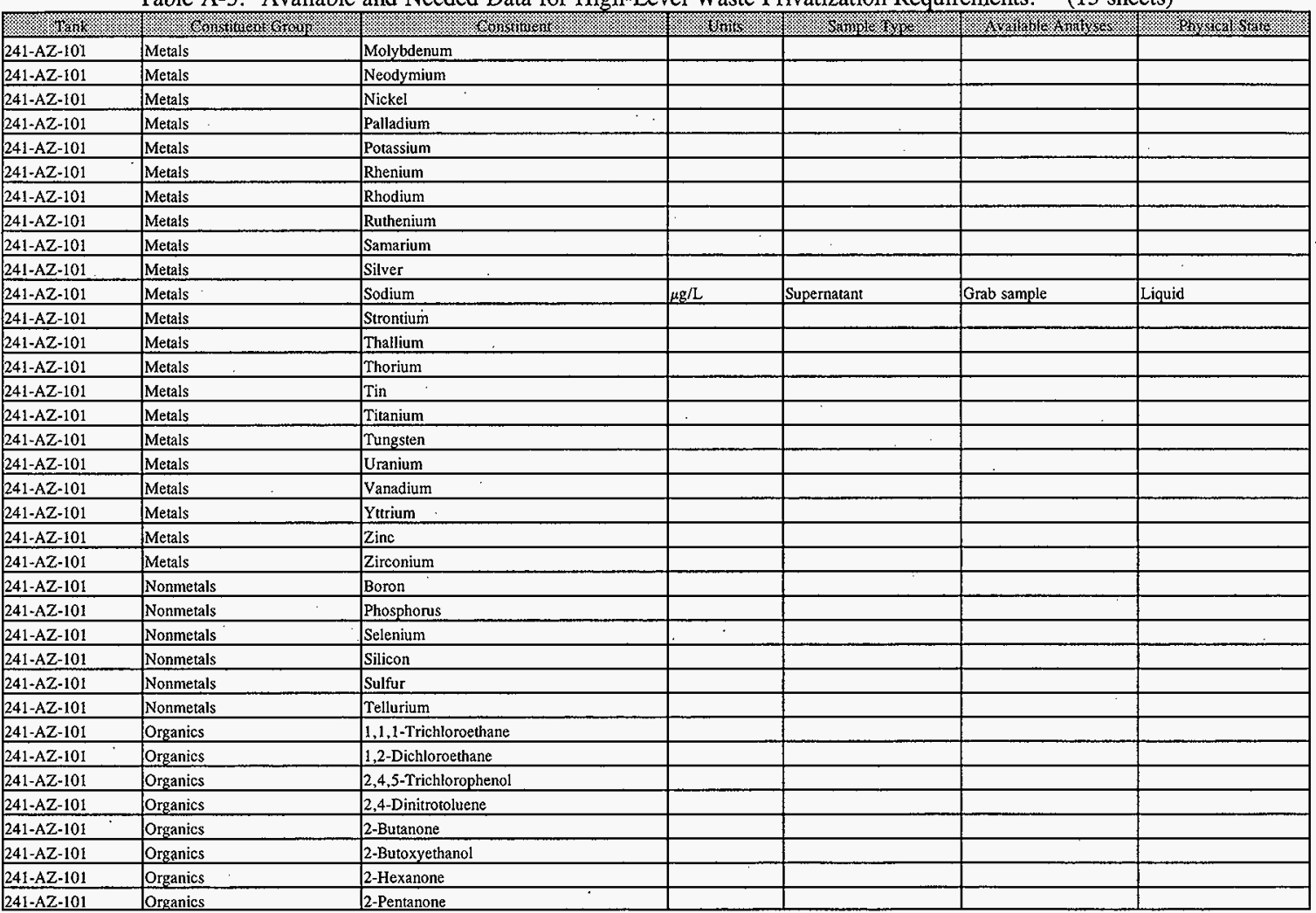


Table A-3. Available and Needed Data for High-Level Waste Privatization Requirements. ${ }^{1.2}$ (13 sheets)

\begin{tabular}{|c|c|c|c|c|c|c|}
\hline V. & (5) & 諼 & 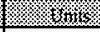 & 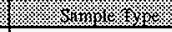 & 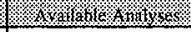 & 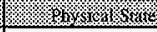 \\
\hline 241-AZ-101 & Organics & 3-Heptanone & & & & \\
\hline $241-A Z-101$ & Organics & Acetone & & & & \\
\hline 241-AZ-101 & Organics & Benzene & & & & \\
\hline $241-\mathrm{AZ}-101$ & Organics & Carbon disulfide & & & & \\
\hline 241-AZ-101 & Organics & Carbon tetrachloride & & & & - \\
\hline $241-\mathrm{AZ}-101$ & Organics & Chloroethane & & & & \\
\hline 241-AZ-101 & Organics & Chloroform & & & & \\
\hline $241-A Z-101$ & Organics & Ethane & & & & \\
\hline 241-AZ-101 & Organics & Ethyl acetate & & & & \\
\hline $241-A Z-101$ & Organics & Ethyl ether & & & & \\
\hline $241-A Z-101$ & Organics & Hexachlorobutadiene & & & & \\
\hline $241-A Z-101$ & Organics & Hexachloroethane & & & & \\
\hline $241-A Z-101$ & Organics & Methylenechloride & & & & \\
\hline 241-AZ-101 & Organics & Naphthalene & & t. & & \\
\hline $241-A Z-101$ & Organics & Nitrobenzene & & & & \\
\hline 241-AZ-101 & Organics & Nonane & & & & \\
\hline $241-A Z-101$ & Organics & $\mathrm{PCB}$ & & & & \\
\hline 241-AZ-101 & Organics & Phenol & & & & \\
\hline 241-AZ-101 & Organics & Pyridine & & 1 & & \\
\hline 241-AZ-101 & Organics & Tetrachloroethene & & & & \\
\hline $241-\mathrm{AZ}-101$ & Organics & Tetrahydrofuran & & & & \\
\hline $241-A Z-101$ & Organics & Toluene & & & & \\
\hline $241-A Z-101$ & Organics & Tributyl phosphate & & & & \\
\hline 241-AZ-101 & Organics & Vinyl chloride & & & & \\
\hline $241-\mathrm{AZ}-101$ & Organics & Xylenes (total) & & & & \\
\hline 241-AZ-101 & Physical Properties & Density & & & & \\
\hline 241-AZ-101 & Physical Properties & DSC (exotherm) & $\%$ & Supernatant & Grab sample & Liquid \\
\hline $241-A Z-101$ & Physical Properties & Particle size density & & & & \\
\hline 241-AZ-101 & Physical Properties & Percent water & $\%$ & Supernatant & Grab sample & Liquid \\
\hline $241-\mathrm{AZ}-101$ & Physical Properties & PH measurement & unitless & Supernatant & Grab sample & Liquid \\
\hline 241-AZ-101 & Physical Properties & Settied solids & & & & \\
\hline $241-\mathrm{AZ}-101$ & Physical Properties & Shear strength & & & & \\
\hline 241-AZ-101 & Physical Properties & Solid density & & & & \\
\hline $241-A Z-101$ & Physical Properties & Specific gravity & unitless & Supernatant & Grab sample & Liquid \\
\hline 241-AZ-101 & Physical Properties & Temperature & & SACS & & \\
\hline $241-A Z-101$ & Physical Properties & Total dissolved solids & & & & \\
\hline
\end{tabular}


Table A-3. Available and Needed Data for High-Level Waste Privatization Requirements. ${ }^{1,2}$ (13 sheets)

\begin{tabular}{|c|c|c|c|c|c|c|}
\hline 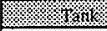 & 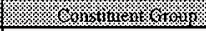 & 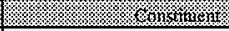 & 3. & V. & 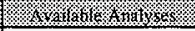 & 9hos \\
\hline $241-A Z-101$ & Physical Properties & Viscosity & & & & \\
\hline $241-A Z-101$ & Physical Properties & Weight percent oxides & & & & \\
\hline $241-A Z-101$ & Physical Properties & Weight percent solids & & & & \\
\hline 241-AZ-101 & Physical Properties & Yield point & & & & \\
\hline 241-AZ-101 & Radionuclides & Americium-241 & $\mu \mathrm{Ci} / \mathrm{L}$ & Supernatant & Grab sample & Liquid \\
\hline 241-AZ-101 & Radionuclides & Americium-243 & & & & \\
\hline 241-AZ-101 & Radionuclides & Antimony-125 & & & & \\
\hline $241-A Z-101$ & Radionuclides & Carbon-14 & & & & - \\
\hline 241-AZ-101 & Radionuclides & Cerium- 144 & & & & \\
\hline $241-A Z-101$ & Radionuclides & Cerium/Praseodymium-144 & & & . & \\
\hline 241-AZ-101 & Radionuclides & Cesium-134 & & & & \\
\hline $241-A Z-101$ & Radionuclides & Cesium-137 & $\mu \mathrm{Ci} / \mathrm{L}$ & Supernatant & Grab sample & Liquid \\
\hline 241-AZ-101 & Radionuclides & Cobalt- 60 & & & & \\
\hline $241-A Z-101$ & Radionuclides & Curium-242 & & & & \\
\hline $241-A Z-101$ & Radionuclides & Curium-243/244 & & & & \\
\hline 241-AZ-101 & Radionuclides & Curium-244 & & & & \\
\hline 241-AZ-101 & Radionuclides & Europium-152 & & & & \\
\hline 241-AZ-101 & Radionuclides & Europium-154 & & & & \\
\hline 241-AZ-101 & Radionuclides & Europium-155 & & & & \\
\hline 241-AZ-101 & Radionuclides & Gross alpha & & & & \\
\hline $241-A Z-101$ & Radionuclides & Gross beta & & & & \\
\hline 241-AZ-101 & Radionuclides & Iodine-129 & & & & \\
\hline $241-A Z-101$ & Radionuclides & Neptunium-237 & & & & \\
\hline 241-AZ-101 & Radionuclides & Nickel-59 & & & & \\
\hline $241-A Z-101$ & Radionuclides & Nickel-63 & & & & \\
\hline 241-AZ-101 & Radionuclides & Plutonium-238 & $\mu \mathrm{Ci} / \mathrm{L}$ & Supernatant & Grab sample & Liquid \\
\hline $241-A Z-101$ & Radionuclides & Plutonium-239 & & & & \\
\hline $241-A Z-101$ & Radionuclides & Plutonium-239/40 & $\mu \mathrm{Ci} / \mathrm{L}$ & Supernatant & Grab sample & Liquid \\
\hline $241-A Z-101$ & Radionuclides & Plutonium-238 & & & & \\
\hline 241-AZ-101 & Radionuclides & Plutonium-239 & & & & \\
\hline 241-AZ-101 & Radionuclides & Plutonium-240 & & & & \\
\hline $241-A Z-101$ & Radionuclides & Plutonium-241 & & & & \\
\hline 241-AZ-101 & Radionuclides & Plutonium-242 & & & & \\
\hline $241-A Z-101$ & Radionuclides & Ruthenium-106 & & & & \\
\hline 241-AZ-101 & Radionuclides & Selenium-79 & & & & \\
\hline $241-A Z-101$ & Radionuclides & Strontium-90 & $\mu \mathrm{Ci} / \mathrm{L}$ & Supernatant. & Grab sample & Liquid \\
\hline
\end{tabular}


Table A-3. Available and Needed Data for High-Level Waste Privatization Requirements. ${ }^{1,2}$ (13 sheets)

\begin{tabular}{|c|c|c|c|c|c|c|}
\hline (1) & - & 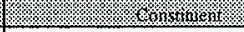 & 16in & 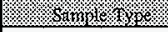 & X & 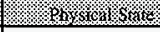 \\
\hline $241-\mathrm{AZ}-101$ & Radionuclides & Technetium-99 & & & & \\
\hline $241-A Z=101$ & Radionuclides & Thorium-228 & & & & \\
\hline 241-AZ-101 & Radionuclides & Tin- 113 & & & & \\
\hline $241-\mathrm{AZ}-101$ & Radionuclides & Total alpha, plutonium & & & & \\
\hline $241-\mathrm{AZ}-101$ & Radionuclides & Tritium & & & & \\
\hline $241-\mathrm{AZ}-101$ & Radionuclides & Uranium-236 & & & & \\
\hline $241-\mathrm{AZ}-101$ & Radionucljdes & Uranium-233 & & & & \\
\hline $241-A Z-101$ & Radionuclides & Uranium-234 & & & & \\
\hline 241-AZ-101 & Radionuclides & Uranium-235 & & & & \\
\hline $241-A Z-101$ & Radionuclides & Uranium-238 & & & & . \\
\hline $241-A Z-102$ & Anions. & Chloride & $\mu \mathrm{g} / \mathrm{L}$ & Supernatant & Grab sample & Liquid \\
\hline $241-\mathrm{AZ}-102$ & Anions & Cyanide & & & & \\
\hline $241-A Z-102$ & Anions & Fluoride & $\mu \mathrm{g} / \mathrm{L}$ & Supernatant & Grab sample & Liquid \\
\hline $241-\mathrm{AZ}-102$ & Anions & Nitrate & $\mu \mathrm{g} / \mathrm{L}$ & Supernatant & Grab sample & Liquid \\
\hline $241-\mathrm{AZ}-102$ & Anions & Nitrite & $\mu \mathrm{g} / \mathrm{L}$ & Supernatant & Grab sample & Liquid \\
\hline $241-\mathrm{AZ}-102$ & Inorganics & Ammonia & & & & \\
\hline $241-A Z-102$ & Inorganics & Carbonate & & & & - \\
\hline 241-AZ-102 & Inorganics & Hydroxide & $\mu \mathrm{g} / \mathrm{L}$ & Supernatant & Grab sample & Liquid \\
\hline $241-\mathrm{AZ}-102$ & Inorganics & Total inorganic carbon & $\mu \mathrm{g} / \mathrm{L}$ & Supernatant & Grab sample & Liquid \\
\hline 241-AZ-102 & Inorganics & Total organic carbon & $\mu \mathrm{g} / \mathrm{L}$ & Supernatant & Grab sample & Liquid \\
\hline $241-\mathrm{AZ}-102$ & Metals & Aluminum & $\mu \mathrm{g} / \mathrm{L}$ & Supernatant & Grab sample & Liquid \\
\hline $241-A Z-102$ & Metals & Antimony & & & & \\
\hline $241-A Z-102$ & Metals & Arsenic & & & & \\
\hline $241-\mathrm{AZ}-102$ & Metals & Barium & & & & \\
\hline $241-\mathrm{AZ}-102$ & Metals & Beryllium & & & & \\
\hline $241-\mathrm{AZ}-102$ & Metals & Bismuth & & & & \\
\hline 241-AZ-102 & Metals & Cadmium & & & & \\
\hline $241-A Z-102$ & Metals & Calcium & & & & \\
\hline 241-AZ-102 & Metals & Cerium & & & & \\
\hline $241-\mathrm{AZ}-102$ & Metals & Cesium & & & & \\
\hline 241-AZ-102 & Metals & Cobalt & & & & \\
\hline $241-A Z-102$ & Metals & Copper & . & & & \\
\hline $241-\mathrm{AZ}-102$ & Metals & Dysprosium & & & . & \\
\hline $241-\mathrm{AZ}-102$ & Metals & Europium & & & & \\
\hline 241-AZ-102 & Metals & Gadolinium & & & & \\
\hline $241-\mathrm{A} Z-102$ & Metals & Iron & $h_{\mu \mathrm{g} / \mathrm{L}}$ & Supernatant & Grab sample & Liquid \\
\hline
\end{tabular}


Table A-3. Available and Needed Data for High-Level Waste Privatization Requirements. 1.2 (13 sheets)

\begin{tabular}{|c|c|c|c|c|c|c|}
\hline \%x. & Kof & Y. & Shis & \%. & \% & \% \\
\hline $241-A Z-102$ & Metals & Lanthanum & & & & \\
\hline $241-A Z-102$ & Metals & Lead & & & & \\
\hline $241-A Z-102$ & Metals & Lithium & & & & \\
\hline 241-AZ-102 & Metals & Magnesium & & & & \\
\hline 241-AZ-102 & Metals & Manganese & & & & \\
\hline $241-\mathrm{AZ}-102$ & Metals & Mercury & & & & \\
\hline 241-AZ-102 & Metals & Molybdenum & & & & \\
\hline $241-A Z-102$ & Metals & Neodymium & & & & \\
\hline 241-AZ-102 & Metals & Nickel & & & $\therefore$ & \\
\hline $241-A Z-102$ & Metals & Palladium & & & & \\
\hline $241-A Z-102$ & Metals & Potassium & & & & \\
\hline $241-\mathrm{AZ}-102$ & Metals & Rhenium & & & & \\
\hline 241-AZ-102 & Metals & Rhodium & & & & \\
\hline $241-A Z-102$ & Metals & Ruthenium & & & & \\
\hline 241-AZ-102 & Metals & Samarium & & & & \\
\hline $241-A Z-102$ & Metals & Silver & & & & \\
\hline $241-A Z-102$ & Metals & Sodium & $\mu \mathrm{g} / \mathrm{L}$ & Supernatant & Grab sample & Liquid \\
\hline $241-A Z-102$ & Metals & Strontium & & & & \\
\hline $241-A Z-102$ & Metals & Thallium & & & & \\
\hline $241-A Z-102$ & Metals & Thorium & & & & \\
\hline $241-A Z-102$ & Metals & Tin & & & & \\
\hline 241-AZ:102 & Metals & Titanium & & & & \\
\hline $241-A Z-102$ & Metals & Tungsten & & & & \\
\hline $241-A Z-102$ & Metals & Uranium & & & & \\
\hline 241-AZ-102 & Metals & Vanadium & & & & \\
\hline $241-A Z-102$ & Metals & Yttrium & & & & \\
\hline $241-A Z-102$ & Metals & Zinc & & & & \\
\hline $241-A Z-102$ & Metals & Zirconium & & & & \\
\hline $241-A Z-102$ & Nonmetals & Boron & & & & \\
\hline $241-A Z-102$ & Nonmetals & Phosphorus & & & & \\
\hline 241-AZ-102 & Nonmetals & Selenium & & & & \\
\hline $241-A Z-102$ & Nonmetals & Silicon & & & & \\
\hline $241-A Z-102$ & Nonmetals & Sulfur & & & & \\
\hline 241-AZ-102 & Nonmetals & Tellurium & & & & . \\
\hline 241-AZ-102 & Organics & 1,1,1-Trichloroethane & & & & \\
\hline $241-\mathrm{AZ}-102$ & Organics & 1,2-Dichloroethane & & & & \\
\hline
\end{tabular}


Table A-3. Available and Needed Data for High-Level Waste Privatization Requirements. ${ }^{1,2}$ (13 sheets)

\begin{tabular}{|c|c|c|c|c|c|c|}
\hline X & 8) & 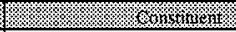 & 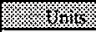 & 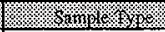 & 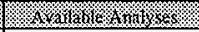 & 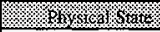 \\
\hline $241-\mathrm{AZ}-102$ & Organics & 2,4,5-Trichlorophenol & & & & \\
\hline $241-A Z-102$ & Orgatics & 2,4-Dinitrotoluene & & & & \\
\hline $241-\mathrm{AZ}-102$ & Organics & 2-Butanone & & & & \\
\hline $241-\mathrm{AZ}-102$ & Organics & 2-Butoxyethanol & & & & \\
\hline $241-\mathrm{AZ}-102$ & Organics & 2-Hexanone & & & & \\
\hline $241-\mathrm{AZ}-102$ & Organics & 2-Pentanone & & & & \\
\hline $241-\mathrm{AZ}-102$ & Organics & Acetone & & & & \\
\hline $241-A Z-102$ & Organies & Bèzene & & & & \\
\hline $241-\mathrm{AZ}-102$ & Organics & Carbon disulfide & & & & \\
\hline 241-AZ-102 & Organics & Carbon terrachloride & & & & \\
\hline 241-AZ-102 & Organics & Chloroethane & & & & \\
\hline 241-AZ-102 & Organics & Chloroform & & & & \\
\hline $241-\mathrm{AZ}-102$ & Organics & Ethane & & & & \\
\hline 241-AZ-102 & Organics & Ethyl acetate & & & & \\
\hline $241-\mathrm{AZ}-102$ & Organics & Ethyl ether & & & & \\
\hline 241-AZ-102 & Organics & Hexachlorobutadiene & & & & \\
\hline $241-\mathrm{AZ}-102$ & Organics & Hexachloroethane & & & & \\
\hline $241-A Z-102$ & Organics & Methylenechloride & & & & \\
\hline $241-\mathrm{AZ}-102$ & Organics & Naphthalene & & & & \\
\hline 241-AZ-102 & Organics & Nitrobenzene & & & & \\
\hline $241-A Z-102$ & Organics & Nonane & & & & \\
\hline $241-A Z-102$ & Organics & $\mathrm{PCB}$ & & & & \\
\hline $241-A Z-102$ & Organics & Phenol & & & & \\
\hline $241-A Z-102$ & Organics & Pyridine & & & & \\
\hline $241-A Z-102$ & Organics & Tetrachloroethene & & & & \\
\hline $241-\mathrm{AZ}-102$ & Organics & Tetrahydrofuran & & & & \\
\hline $241-A Z-102$ & Organics & Toluene & & & & \\
\hline $241-A Z-102$ & Organics & Vinyl chloride & & & & \\
\hline $241-A Z-102$ & Organics & Xylenes (total) & & & & \\
\hline $241-\mathrm{AZ}-102$ & Physical Properties & Density & & & & \\
\hline $241-A Z-102$ & Physical Properties & DSC (exotherm) & $\%$ & Supernatant & Grab sample & Liquid \\
\hline $241-\mathrm{AZ}-102$ & Physical Properties & Particle size density & & & & \\
\hline $241-\mathrm{AZ}-102$ & Physical Properties & Percent water & $\%$ & Supernatant & Grab sample & Liquid \\
\hline $241-\mathrm{AZ}-102$ & Physical Properties & PH measurement & unitless & Supernatant & Grab sample & Liquid \\
\hline $241-A Z-102$ & Physical Properties & Settled solids & & & & \\
\hline $241-\mathrm{AZ}-102$ & Physical Properties & Shear strength & & & & \\
\hline
\end{tabular}


Table A-3. Available and Needed Data for High-Level Waste Privatization Requirements. ${ }^{1,2}$ (13 sheets)

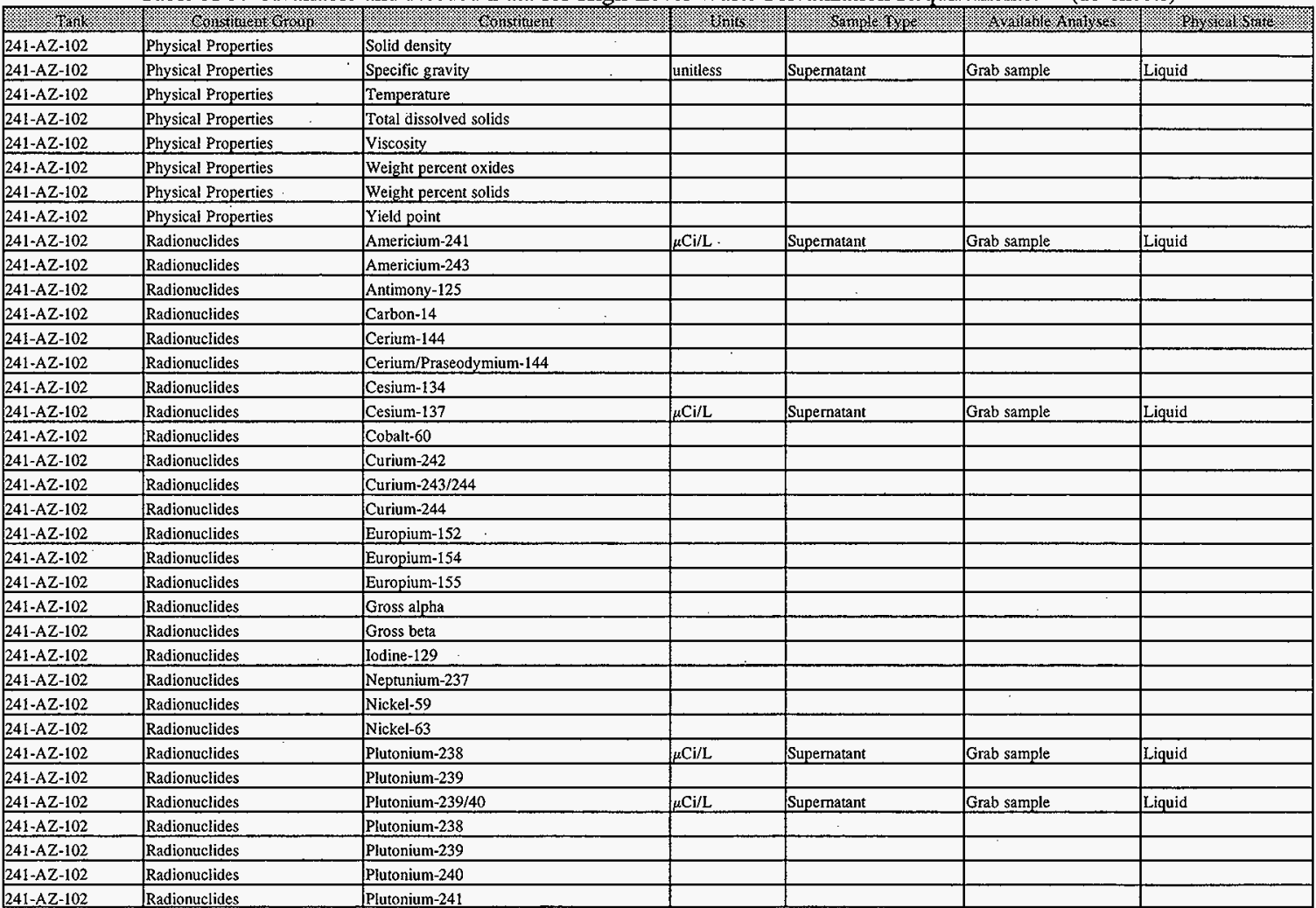


Table A-3. Available and Needed Data for High-Level Waste Privatization Requirements. ${ }^{1,2}$ (13 sheets)

\begin{tabular}{|c|c|c|c|c|c|c|}
\hline 16 & Vhinstiule & W & 椎 & 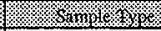 & 13 & ffirsical \\
\hline 241-AZ-102 & Radionuelides & Plutonium-242 & & & & \\
\hline 241-AZ-102 & Radionuclides & Ruthenium-106 & & & & \\
\hline 241-AZ-102 & Radionuclides & Selenium-79 & & & & \\
\hline 241-AZ-102 & Radionuclides & Strontium-90 & $\mu \mathrm{Ci} / \mathrm{L}$ & Supernatant & Grab sample & Liquid \\
\hline 241-AZ-102 & Radionuclides & Technetium-99 & & & & \\
\hline $241-A Z-102$ & Radionuclides & Thorium-228 & & & & \\
\hline 241-AZ-102 & Radionuclides & Tin-113 & & & $\cdot$ & \\
\hline 241-AZ-102 & Radionuclides & Total alpha, plutonium & & & & \\
\hline 241-AZ-102 & Radionuclides & Tritium & & & & \\
\hline $241-A Z-102$ & Radionuclides & Uranium-236 & & & & \\
\hline $241-\mathrm{AZ}-102$ & Radionuclides & Uranium-233 & & & & \\
\hline $241-\mathrm{AZ}-102$ & Radionuclides & Uranium-234 & & & & \\
\hline $241-A Z-102$ & Radionuclides & Uranium-235 & & & & \\
\hline $241-A Z-102$ & Radionuclides & Uranium-238 & & & & \\
\hline
\end{tabular}

Notes:

${ }^{t}$ Requirements and available data are shown for each LAW and HLW tank in scope. No data is available where columns 4, 5, 6, and 7 are blank.

${ }^{2}$ Analytes listed in column 3 are those included in the Tank Characterization Database. This table does not include requirements not listed in TCD.

$\mu \mathrm{g} / \mathrm{L} \quad=\quad$ micrograms per liter

$\% \quad$ p percent

= microcuries per liter 
HNF-2117 Rev. 0

This page intentionally left blank. 
HNF-2117 Rev. 0

APPENDIX B

TANKK SAMPLING PLANS 
HNF-2117 Rev. 0

This page intentionally left blank. 
Table B-1. Tank Sampling Plans as of October 10, 1997. (4 sheets)

\begin{tabular}{|c|c|c|c|}
\hline Shtank & 6. & 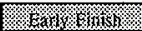 & 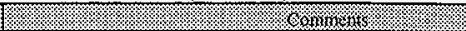 \\
\hline 241-AN-102 Grab Sample Privatization - Wiemers & 12-Dec-97 & 16-Dec-97 & \\
\hline 241-AN-102 Grab Sample Compatability Batch 9 (NCF) & 5-Jul-06 & 6-Iul-06 & \\
\hline 241-AN-102 Grab Sample Process Control Batch 9 (NCF) & 1-Aug-06 & 1-Aug-06 & \\
\hline 241-AN-102 Grab Sample Process Control Batch 9 (NCF) & $1-\operatorname{Sep}-06$ & 5-Sep-06 & \\
\hline 241-AN-103 Grab Sample Compatability Batch 5 (NCF) & 04-Sep-01 & 05-Sep-01 & \\
\hline 241-AN-103 Grab Sample Process Control Batch 5 (NCF) & 01-Nov-04 & 02-Nov-04 & \\
\hline 241-AN-103 Grab Sample Process Control Batch 5 (NCF) & 01-Dec-04 & 02-Dec-04 & \\
\hline 241-AN-104 Grab Sample Compatability Batch 3 (NCF) & $03-F e b-03$ & 04-Feb-03 & \\
\hline 241-AN-104 Grab Sample Process Control Batch 3 (NCF) & $01-$ Apr-03 & $02-A p r-03$ & - \\
\hline 241-AN-104 Grab Sample Process Control Batch 3 (NCF) & 01-May-03 & 02-May-03 & \\
\hline 241-AN-105 Grab Sample Compability Batch 1 (NCF) & 01-Feb-01 & 02-Feb-01 & \\
\hline 241-AN-105 Grab Sample Process Control Batch 1 (NCF) & $02-\mathrm{Apr}-01$ & 03-Apr-01 & $\dot{.}$ \\
\hline 241-AN-105 Grab Sample Process Control Batch 1 (NCF) & 01-May -01 & 02-May-01 & \\
\hline 241-AN-106 Push Sample 2 Segments 5 & 27-Mar-00 & 21-Apr-00 & Requires tank full for privatization \\
\hline 241-AN-106 Grab Sample Compatability Batch 10 (NCF) & 01-Dec-06 & 04-Dec-06 & \\
\hline 241-AN-106 Grab Sample Process Control Batch 10 (NCF) & $01-F e b-07$ & 02-Feb-07 & \\
\hline 241-AN-106 Grab Sample Process Control Batch 10 (NCF) & 01-Mar-07 & $02-\mathrm{Mar}-07$ & \\
\hline 241-AN-106 Grab Sample Compatability Batch 11 (NCF) & 03-Aug.07 & 06-Aug-07 & \\
\hline 241-AN-106 Grab Sample Process Control Batch 11 (NCF & 01-Nov-07 & 02-Nov-07 & \\
\hline 241-AN-106 Grab Sample Process Control Batch 11 (NCF) & 15-Nov-07 & 16-Nov-07 & \\
\hline 241-AN-107 Push Sample 2 Segments 22 & 28-Jan-00 & 25-Feb-00 & Requires tank full for privatization, requires $\mathrm{pH}$ adjustment. \\
\hline 241-AN-107 Grab Sample Compatability Batch 7 (NCF) & $01-\mathrm{Feb}-06$ & $02-F e b-06$ & \\
\hline 241-AN-107 Grab Sample Process Control Batch 7 (NCF) & 15-Feb-06 & 16-Feb-06 & \\
\hline 241-AN-107 Grab Sample Process Control Batch 7 (NCF) & $01-\mathrm{Mar}-06$ & 02 -Mar-06 & \\
\hline 241-AP-102 Grab Sample Process Control Batch 1 (NCF) & 04-Apr-01 & O5-Apr-01 & \\
\hline 241:AP-102 Grab Sample Process Control Batch 1 (NCF) & 03-May-01 & 04-May-01 & \\
\hline 241-A.P-102 Grab Sample Qualification Batch 1 (NCF) & 01-Jun-01 & $04-J u n-01$ & \\
\hline 241-AP-102 Grab Sample Process Control Batch 2 (NCF) & 01-Nov-01 & 02-Nov-01 & \\
\hline 24L-AP-102 Grab Sample Process Control Batch 2 (NCF) & $01-0 \mathrm{ct}-02$ & $02-0 \mathrm{ct}-02$ & \\
\hline 241-AP-102 Grab Sample Process Control Batch 2 (NCF) & 15-Oct-02 & $16-$ Oct-02 & \\
\hline 24l-AP-102 Grab Sample Qualification Batch 2 (NCF) & $01-\mathrm{Nov}-02$ & 04-Nov-02 & \\
\hline 24l-AP-102 Grab Sample Process Control Batch 3 (NCF) & 03-Apr-03 & $04-A \mathrm{pr}-03$ & \\
\hline 241-AP-102 Grab Sample Process Control Batch 3 (NCF) & 05-May-03 & 06-May-03 & \\
\hline 241-AP-102 Grab Sample Qualification Batch 3 (NCF) & $03-\mathrm{Jun}-03$ & 04-Jun-03 & \\
\hline 241-AP-102 Grab Sample Process Control Batch 4 (NCF) & 09-Jan-04 & 12-Jan-04 & \\
\hline 241-AP-102 Grab Sample Process Control Batch 4 (NCF) & 02-Feb-04 & 03-Feb-04 & \\
\hline 241-AP-102 Grab Sample Qualification Batch 4 (NCF) & $01-\mathrm{Mar}-04$ & 02-Mar-04 & \\
\hline 241-AP-102 Grab Sample Process Control Batch 5 (NCF) & 03-Dec-04 & 06-Dec-04 & \\
\hline
\end{tabular}


Table B-1. Tank Sampling Plans as of October 10, 1997. (4 sheets)

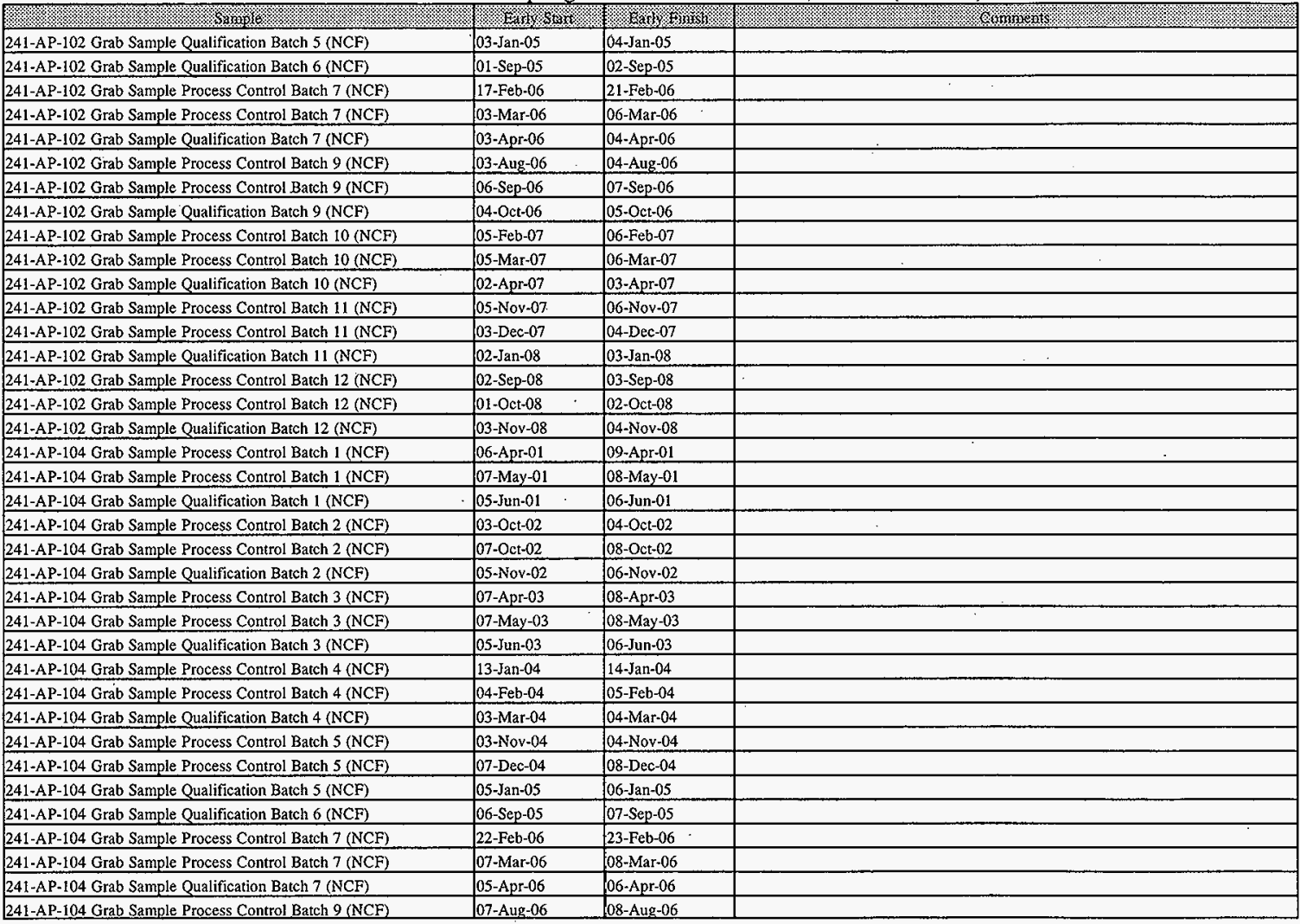


Table B-1. Tank Sampling Plans as of October 10, 1997. (4 sheets)

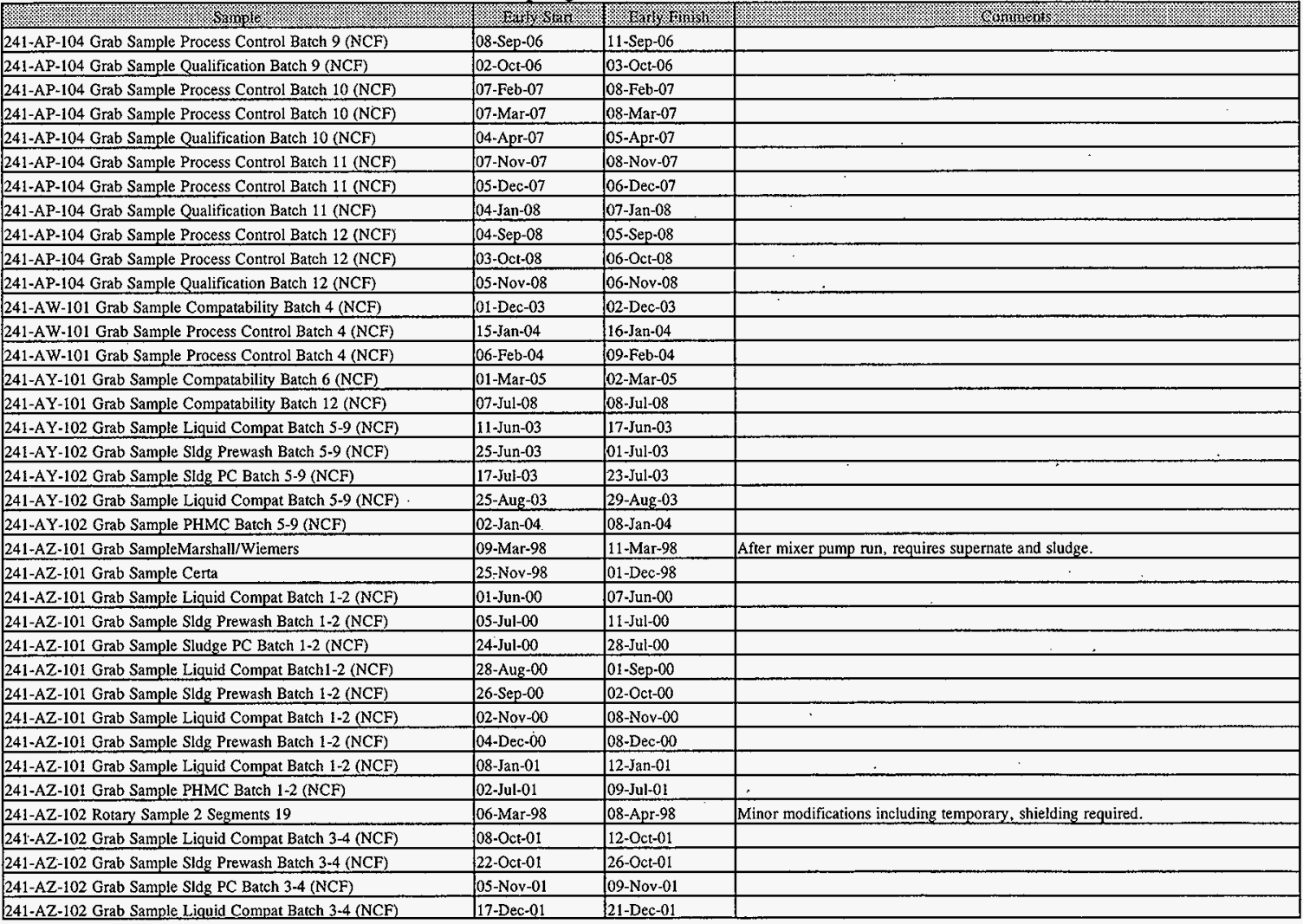


Table B-1. Tank Sampling Plans as of October 10, 1997. (4 sheets)

\begin{tabular}{|c|c|c|c|}
\hline S & Kaxpin & KL & S \\
\hline 241-AZ-102 Grab Sample Sldg PC Batch 3-4 (NCF) & 16-Jan-02 & 22-Jan-02 & \\
\hline 241-AZ-102 Grab Sample Liquid Compat Batch 3-4 (NCF) & 22-Feb-02 & $28-\mathrm{Feb}-02$ & \\
\hline 241-AZ-102 Grab Sample Sldg Prewash Batch 3-4 (NCF) & 25-Mar-02 & 29-Mar-02 & \\
\hline 241-AZ-102 Grab Sample Liquid Compat Batch 3-4 (NCF) & $30-A p r-02$ & 06-May-02 & \\
\hline 241-AZ-102 Grab Sample PHMC Batch 3-4 (NCF) & $24-\mathrm{Oct}-02$ & $30-\mathrm{Oct}-02$ & \\
\hline 241-SY-103 Grab Sample - Privatization - Wiemers & 09-Apr-98 & 13-Apr-98 & \\
\hline 241-SY-103 Push Samples 1 Segments 15 & 23-Feb-01 & 15-Mar-01 & Requires Unresolved Safety Question Review for Group 1 Tank - Co \\
\hline
\end{tabular}

Notes:

$\begin{array}{lll}\text { Compat } & = & \text { Compatibility } \\ \text { NCF } & = & \text { Not currently funded } \\ \text { PC } & = & \text { process control } \\ \text { PHMC } & = & \text { Project Hatnford Management Contractor } \\ \text { Sldg } & = & \text { Sludge }\end{array}$




\begin{tabular}{|c|c|c|c|c|c|c|}
\hline \multicolumn{7}{|c|}{ DISTRIBUTION SHEET } \\
\hline \multirow[b]{2}{*}{ Distribution } & \multirow{2}{*}{\multicolumn{4}{|c|}{$\begin{array}{l}\text { From } \\
\text { Technical Basis and Planning }\end{array}$}} & \multicolumn{2}{|l|}{ Page 1 of 1} \\
\hline & & & & & \multicolumn{2}{|c|}{$\begin{array}{ll}\text { Date } & 01 / 19 / 98\end{array}$} \\
\hline \multicolumn{5}{|l|}{ Project Title/Work Order } & \multicolumn{2}{|c|}{ EDT No. EDT-622419 } \\
\hline \multicolumn{5}{|c|}{$\begin{array}{l}\text { HNF-2117, Rev. } 0 \text {, "Readiness to Proceed: Characterization } \\
\text { Planning Basis" }\end{array}$} & \multicolumn{2}{|l|}{ ECN No. N/A } \\
\hline \multicolumn{2}{|l|}{ Name } & MSIN & $\begin{array}{c}\text { Text } \\
\text { With } \\
\text { A17 } \\
\text { Attach. }\end{array}$ & Text Only & $\begin{array}{l}\text { Attach./ } \\
\text { Appendix } \\
\text { Only }\end{array}$ & $\begin{array}{l}\text { EDT/ECN } \\
\text { Only }\end{array}$ \\
\hline $\begin{array}{l}\text { Lockheed Martin Hanford Corp. } \\
\text { M. R. Adams } \\
\text { J. M. Conner } \\
\text { J. G. Field } \\
\text { E. I. Husa } \\
\text { J. Jo } \\
\text { M. J. Kupfer } \\
\text { T.C.S.R.C. }\end{array}$ & & $\begin{array}{l}\text { R2 }-12 \\
\text { R2-11 } \\
\text { R2-12 } \\
\text { R2-11 } \\
\text { R2-12 } \\
\text { H5 }-49 \\
\text { R1-10 }\end{array}$ & $\begin{array}{l}x \\
x \\
x \\
x \\
x \\
x \\
x \\
x\end{array}$ & & & \\
\hline $\begin{array}{l}\text { Lockheed Martin Services, Inc. } \\
\text { Central Files }\end{array}$ & & B1-07 & $x$ & & & \\
\hline$\frac{\text { Technical Resources, Internatior }}{\text { L. J. Fergestrom }}$ & Inc. & $\mathrm{R} 2-12$ & $x$ & & & \\
\hline
\end{tabular}

